Argonne

\title{
Accelerator-Driven Subcritical System for Disposing of the U.S. Spent Nuclear Fuel Inventory
}

Nuclear Science \& Engineering Division 


\section{*Custom Text Heading}

Custom Text

About Argonne National Laboratory

Argonne is a U.S. Department of Energy laboratory managed by UChicago Argonne, LLC under contract DE-AC02-06CH11357. The Laboratory's main facility is outside Chicago, at 9700 South Cass Avenue, Argonne, Illinois 60439. For information about Argonne and its pioneering science and technology programs, see www.anl.gov.

\section{DOCUMENT AVAILABILITY}

Online Access: U.S. Department of Energy (DOE) reports produced after 1991 and a growing number of pre-1991 documents are available free at OSTI.GOV (http://www.osti.gov/), a service of the US Dept. of Energy's Office of Scientific and Technical Information.

Reports not in digital format may be purchased by the public from the National Technical Information Service (NTIS):

U.S. Department of Commerce

National Technical Information Service

5301 Shawnee Rd

Alexandria, VA 22312

www.ntis.gov

Phone: (800) 553-NTIS (6847) or (703) 605-6000

Fax: (703) 605-6900

Email: orders@ntis.gov

Reports not in digital format are available to DOE and DOE contractors from the

Office of Scientific and Technical Information (OSTI):

U.S. Department of Energy

Office of Scientific and Technical Information

P.O. Box 62

Oak Ridge, TN 37831-0062

www.osti.gov

Phone: (865) 576-8401

Fax: (865) 576-5728

Email: reports@osti.gov 



\section{Accelerator-Driven Subcritical System for Disposing of the U.S. Spent Nuclear Fuel Inventory}

prepared by

Yousry Gohar, Yan Cao, and Adam R. Kraus

Nuclear Science \& Engineering Division, Argonne National Laboratory

Prepared for Argonne National Laboratory's work was supported by the U.S. Department of Energy, Office of Science, under Contract No. DE-AC02-06CH11357.

April 2018 


\section{Accelerator-Driven Subcritical System for Disposing of the U.S. Spent Nuclear Fuel Inventory}

Contents

Page

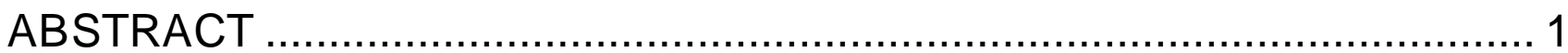

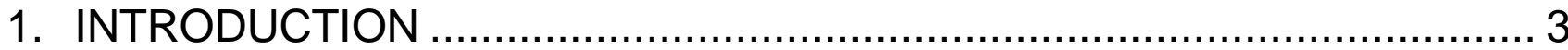

2 PHYSICS ANALYSIS OF THE HOMOGENEOUS CONFIGURATION...... 5

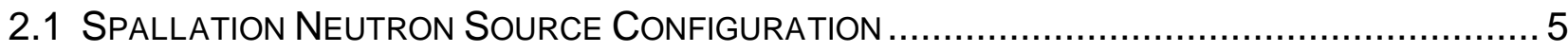

2.2 SUBCRITICAL FISSION BLANKET DESIGN .............................................................

3 PRELIMINARY FUEL BURNUP ANALYSIS OF THE ADS SYSTEM ..... 10

4 ADS CONCEPTUAL CONFIGURATIONS …….................................... 14

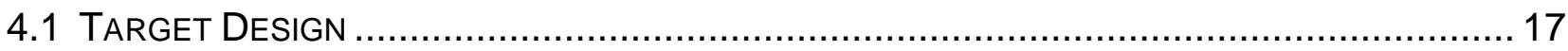

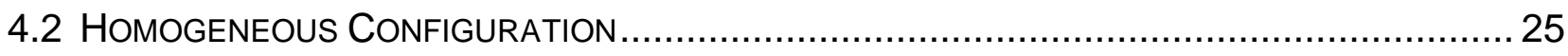

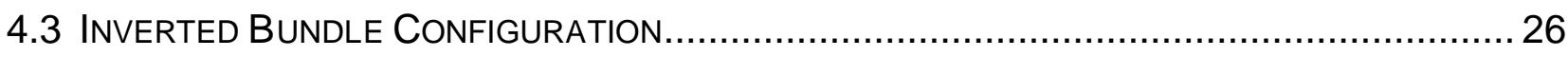

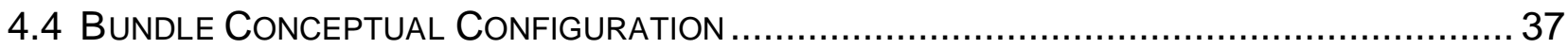

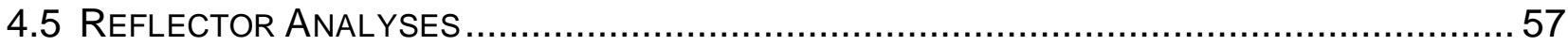

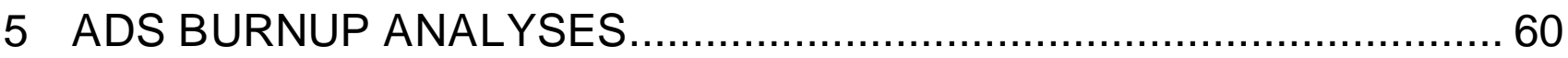

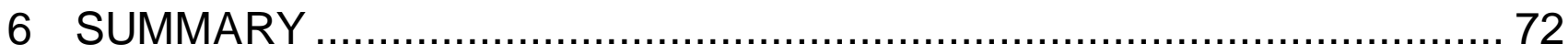

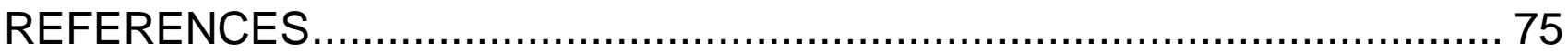




\section{Accelerator-Driven Subcritical System for Disposing of the U.S. Spent Nuclear Fuel Inventory}

Figures

Page

Figure 1. ADS system model (White: proton beam channel; Green: LBE target/coolant material; Blue: HT-9 wall; Yellow: LBE fuel blanket; Red: graphite)

Figure 2. LBE total energy deposition as function of target length for various proton energies

Figure 3. The fission blanket infinite neutron multiplication as a function the U-Pu concentration in the actinide material for different actinide concentrations in the LBE fuel carrier

Figure 4. The fission blanket effective neutron multiplication as a function of the plutonium concentration in the actinide fuel material with $7 \%$ actinides loaded in the LBE fuel carrier, and the corresponding total ADS power using $25 \mathrm{MW}$ beam power delivered by $1 \mathrm{GeV}$ protons as function of the $\mathrm{k}_{\text {eff }}$ value.

Figure 5. The total MA inventory and the fission blanket volume as a function of the plutonium concentration in the minor actinide material for different actinide material concentrations in the LBE fuel carrier, all blankets with an effective neutron multiplication factor of 0.98

Figure 6. Am-241 $(\mathrm{n}, \mathrm{g}) \mathrm{Am}-242 \mathrm{~g}$ branching ratios from Mann \& Schneter calculation, ENDF/B-VI.8, -VII.0 nuclear data files, and CINDER90 library.

Figure 7. Effective neutron multiplication value before and after each fuel burnup time step for the three fission blanket configurations of Table II

Figure 8. Actinides and LLFPs annual feeding rates at end of each burnup step for blanket configuration with 5\% actinide concentration in LBE and $35.7 \%$ plutonium concentration in actinides

Figure 9. Actinides and LLFPs annual feeding rates at end of each burnup step for the blanket configuration with $7 \%$ actinide concentration in LBE and $27.2 \%$ plutonium concentration in actinides...

Figure 10. Actinides and LLFPs annual feeding rates at end of each burnup step for the blanket configuration with $10 \%$ actinide concentration in LBE and $20 \%$ plutonium concentration in actinides.

Figure 11. Total actinides, U-Pu, and MAs transmutation rates for three blanket configurations of Table II 
Figure 12 Spallation neutron target model for the ADS system (White: proton beam; Blue: target material; Red: HT-9 structure wall).

Figure 13 Full (left) and zoomed (right) views of the target assembly, with the coolant flow path illustrated. Gray is the beam tube, yellow is the inlet/outlet separation wall, green is the coolant-bounding wall, and blue is the target guard vessel.

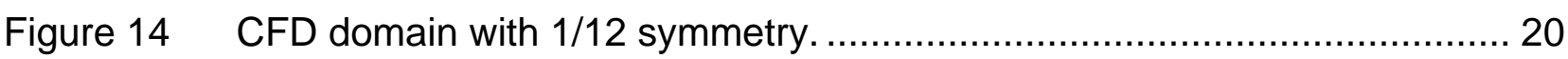

Figure 15 Power density $\left(\mathrm{W} / \mathrm{m}^{3}\right)$ for all target materials....................................... 21

Figure 16 Velocity magnitude distribution at the symmetry plane for the base case. . 22

Figure 17 Temperature distribution at the symmetry plane for the base case............ 22

Figure 18 Structural temperatures at the symmetry plane for the base case............ 23

Figure 19 Velocity magnitude distribution at the symmetry plane for the Standard k-

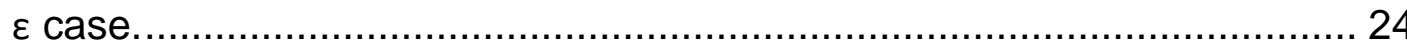

Figure 20 Monte Carlo Model of the Homogeneous Self-Cooled Blanket

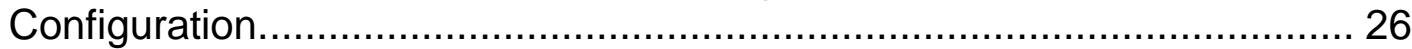

Figure 21 (a) $X-Z$ view and (b) $X-Y$ views of the Monte Carlo simulation model of the subcritical assembly of the inverted bundle design concept.

Figure 22 (a) The radial power density distribution at the middle plane and $\pm 10 \mathrm{~cm}$ below or above the middle plane (b) The axial power density distribution at the radial positions of $35.0,45.5,75.5,155.0$, and $185.0 \mathrm{~cm}$.

Figure 23 Energy deposition maps in the fuel, tube walls, and coolant for one wedge of the second subcritical assembly configuration in $\mathrm{kW} / \mathrm{cm}^{3}$.

Figure 24 Minimal symmetry unit used in CFD simulations with some boundary conditions labeled.

Figure $25 \quad$ CFD mesh for the minimal symmetry unit. ........................................... 33

Figure 26 Sample temperature distribution for the minimal symmetry unit................ 33

Figure 27 Peak cladding temperature distribution across the domain. Tube number increases with increasing radial coordinate. Case $A$ is the optimal configuration.

Figure 28 Radial temperature distribution for two cases with different tube radii. Note the higher power but comparable temperatures for the $7 \mathrm{~mm}$ case.

Figure 29 Differing meshes for power mapping. Red is the MCNPX uniform mesh (for illustration only, not to scale), gray and green are the unstructured CFD mesh.

Figure 30 Power profiles mapped onto the CFD mesh for fuel, clad, and coolant regions.

Figure 31 Peak cladding surface temperature comparison for cases with and without heating in the coolant and cladding included. 
Figure 32 ISO Temperature surfaces for the full power, optimal case. Clockwise from left: $873 \mathrm{~K}, 973 \mathrm{~K}$, and $1173 \mathrm{~K}$.

Figure 33 Calculated maximum surface temperature of each coolant tube for both design concepts.

Figure 34 Vertical and horizontal cross section of the bundle conceptual configuration consists of one eighth of the subcritical fission blanket.

Figure 35 Axial power density distributions of the selected four fuel tubes shown in Figure 34(b) of the conceptual bundle configuration.

Figure 36 CFD Geometric model for simulating two rows of fuel tubes. Top and bottom boundaries are symmetry planes; left and right boundaries are adiabatic walls.

Figure 37 Small section of finite volume mesh used in CFD model, displaying fuel (brown), clad (blue), and coolant (gray) regions.

Figure 38 Zoomed view of velocity magnitude on an axial symmetry plane.

Figure 39 Radial component of velocity distribution.

Figure 40 Cladding inner surface radial temperature distribution.

Figure 41 Cladding inner surface radial temperature distribution exceeding the 873 $\mathrm{K}$ temperature limit.

Figure 42 Maximum clad surface temperature as a function of the fuel tube center coordinate. Temperature jump upward at $\sim 100 \mathrm{~cm}$ is due to the fuel tube diameter change.

Figure 43 Cladding inner surface radial temperature distribution for the updated fission blanket.

Figure 44 Cladding inner surface radial temperature distribution of the updated fission blanket exceeding the $873 \mathrm{~K}$ temperature limit.

Figure 45 Vertical and mid-plane views of the bundle concept of the fission blanket. 47

Figure 46 Mid-plane temperature from obtained from the inner model calculation. The purple circle denotes the fuel tube with the peak power/temperature. The gray box contains the fuel tubes used in the strip model.

Figure 47 Mid-plane temperature obtained from the strip model calculation. Bottom symmetry plane is coincident with the sector centerline. The gray box contains the tubes used in the inner model.

Figure 48 Comparison of mid-plane velocity distribution for the selected configuration (left) and for the same configuration with dummy tubes (right).

Figure 49 Inlet velocity distributions for the 3-zone (top), 4-zone (middle), and line (bottom) cases.

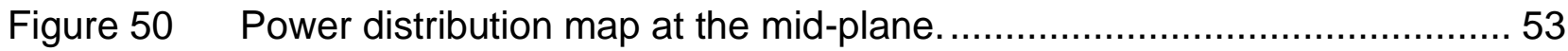

Figure $51 \quad$ Axial fuel power distribution at the symmetry plane ............................... 53 
Figure 52 Mid-plane temperature distribution for the coolant and cladding, highlighting the tubes with surface temperature above $870 \mathrm{~K} \ldots \ldots \ldots \ldots \ldots \ldots . . . . . . . .54$

Figure 53 Mid-plane temperature distribution of all materials............................... 54

Figure 54 Axial temperature distribution at the symmetry plane of all materials ....... 55

Figure 55 Tube surface temperatures above $850 \mathrm{~K}$ are highlighted with pink. These tubes are located near the tube diameter transitions. ...............................56

Figure $56 \quad$ Axial velocity profile at the mid-plane.............................................. 57

Figure 57 Calculated energy depositions in the homogeneous fission blanket with a graphite reflector.

Figure 58 Neutron multiplication factor of the homogeneous concept as function of the reflector thickness in the radial and axial directions.

Figure 59 Energy spectrum of the spallation neturons at their born sites and at the outer target surface.

Figure 60 Calculated effective neutron multiplication values $\left(k_{\text {eff }}\right)$ before and after each fuel burnup time step of the ADS bundle configuration from SERPENT calculations using the surface neutron source.

Figure 61 Actinide transmutation rates of the ADS bundle configuration from SERPENT calculations using the surface and the volume neutron source.

Figure 62 Generated and actual inventories of the long-lived fission products in the ADS mobile fuel as a function of the full power years.

Figure 63 The actinide fuel compositions in the mobile fuel of the ADS bundle configuration after different operating periods.

Figure 64 The actinide fuel inventory and the total actinide fed into the subcritical fission blanket as a function of the full power years.

Figure 65 Comparison of the calculated transmutation rates from the SERPENT and the MCB5 simulations using the volume source for the ADS bundle configuration.

Figure 66 Comparison of the consumed actinides calculated from the SERPENT and the MCB5 simulations using the volume source for the ADS bundle configuration.

Figure 67 Comparison of the controlled $k_{\text {eff }}$ and the integrated amount of actinide fuel fed from the SERPENT and the MCB5 simulations using the volume source for the ADS bundle configuration.

Figure 68 Comparison of the LLFPs from the SERPENT and the MCB5 simulations of the ADS bundle configuration using the volume source and operating for 35 full power years 


\title{
Accelerator-Driven Subcritical System for Disposing of the U.S. Spent Nuclear Fuel Inventory
}

\author{
Tables
}

$\underline{\text { Page }}$

Table I. Main parameters of the LBE target designs. $\quad$....................................... 7

Table II. Three fission blanket configurations with $0.98 \mathrm{k}_{\text {eff }}$ and MA inventory $\leq 10$

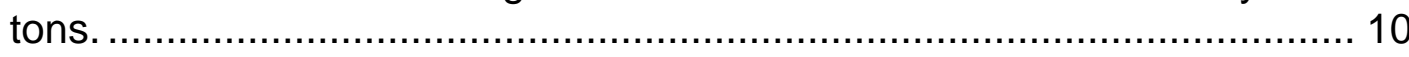

Table III. Main parameters of the neutron target designs using liquid $\mathrm{Pb}$ or LBE. .... 17

Table IV Comparison of key thermal hydraulic values with mesh refinement, Temperatures in $\mathrm{K}$, pressures in $\mathrm{KPa}$.

Table V Comparison of key thermal hydraulic values for various test cases, temperatures in $\mathrm{K}$, pressures in $\mathrm{kPa}$................................................ 24

Table VI Physical parameters of the homogenous configuration ........................... 27

Table VII. Main parameters of the inverted bundle configuration ............................. 28

Table VIII Geometry and flow parameters for the full power CFD simulation............. 36

Table IX Main physical parameters of the bundle conceptual configuration............. 39

Table $X \quad$ Main parameters of MAs conceptual design using the bundle model. ....... 40

Table XI Comparison of key thermo-fluid system performance parameters for the eight-sector and six-sector cases.

Table XII Thermal-hydraulic performance comparison for various inlet velocity profiles.

Table XIII. Main parameters of the conceptual design using the bundle model. 58

Table XIV. Comparison of the total neutron fluxes of the bundle conceptual configuration calculated with MCNP6, SERPENT, and MCB5 codes using point neutron source with different energies.

Table XV. Serpent transmutation rates of the actinides using the ADS conceptual configuration operating for 35 full power years ........................................6 65

Table XVI Serpent calculated transmutation of the LLFPs in the ADS conceptual configuration using the bundle model within 35 years of operation. 65

Table XVII. Transmuted actinides from the MCB5 and the SERPENT simulations of the ADS bundle configuration operating for 35 full power years. 


\section{Accelerator-Driven Subcritical System for Disposing of the U.S. Spent Nuclear Fuel Inventory}

\section{Abstract}

The current United States inventory of the spent nuclear fuel (SNF) is 80,000 metric tons of heavy metal (MTHM), including 131 tons of minor actinides (MAs) and $\sim 669$ tons of plutonium. This study describes a conceptual design of an accelerator-driven subcritical (ADS) system for disposing of this SNF inventory by utilizing the 131 tons of MAs inventory and a fraction of the plutonium inventory for energy production, and transmuting some long-lived fission products.

An ADS system with a homogeneous subcritical fission blanket was first examined. A spallation neutron source is used to drive the blanket and it is produced from the interaction of a 1-GeV proton beam with a lead-bismuth eutectic (LBE) target. The blanket has a liquid mobile fuel using LBE as the fuel carrier. The fuel materials are dissolved, mixed, or suspended in the liquid fuel carrier. Monte Carlo analyses were performed to determine the overall parameters of the concept. Steady-state Monte Carlo simulations were performed for three similar fission blankets. Except for, the loaded amount of actinide materials in the LBE is either 5,7 , or $10 \%$ of the total volume of the blanket, respectively. The neutron multiplication factors of the three blankets are $\sim 0.98$ and the initial MAs blanket inventories are $\sim 10$ tons. In addition, Monte Carlo burnup simulations using the MCB5 code were performed to analyze the performance of the three conceptual ADS systems. During operation, fresh fuel was fed into the fission blanket to adjust its reactivity and to control the system power. The burnup analysis shows that the three ADS concepts consume about 1.2 tons of actinides per full power year and produce $3 \mathrm{GW}$ thermal power, with a proton beam power of $25 \mathrm{MW}$. For the blankets with 5, 7, or 10\% actinide fuel particles loaded in the LBE, assuming that the ADS systems can be operated for 35 full-power years, the total MA materials consumed in the three ADS systems are about 30.6, 35.3, and 37.2 tons, respectively. Thus, the corresponding numbers of ADS systems to utilize the 131 tons of MA materials of the SNF inventory are 4.3, 3.7, or 3.5, respectively.

ADS concepts with tube bundles inserted in the fission blanket were analyzed to overcome the disadvantages of the homogeneous blanket concept. The liquid lead is used as the target material, the mobile fuel carrier, and the primary coolant to avoid the polonium production from bismuth. Reactor physics and thermal-hydraulic analyses were coupled to determine the parameters of the heterogeneous fission blanket. The engineering requirements for a satisfactory operation performance of the HT-9 ferritic steel structure material have been realized. Two heterogeneous concepts of the subcritical fission blanket with the liquid lead mobile fuel inside or outside the tube bundles were considered. The heterogeneous configuration with the mobile fuel inside the tubes showed better performance than the configuration with mobile fuel outside the bundle tubes. The Monte Carlo burnup codes, MCB5 and SERPENT were both used to simulate 
the fuel burnup in the ADS concepts with the mobile fuels inside the tubes. The burnup analyses were carried out for 35 full power years. The results show that 5 ADS systems can dispose of the total United States inventory of the spent nuclear fuel. 


\section{Accelerator-Driven Subcritical System for Disposing of the U.S. Spent Nuclear Fuel Inventory}

\section{Introduction}

In the U.S. Department of Energy (DOE) environmental impact studies of the Yucca Mountain nuclear waste repository [1], the spent nuclear fuels (SNF) from nuclear power and other reactors were examined. Based on this study, the current United States inventory of SNF is estimated to be $\sim 80,000$ metric tons of heavy metal (MTHM), including $\sim 131$ tons of minor actinides (MAs) and $\sim 669$ tons of plutonium.

The compositions of the discharged spent nuclear fuel from the light water power reactors (LWRs) differ from one reactor to another due to differences in the initial fuel composition, irradiation history, and the cooling time [2]. However, the total amount of transuranic elements is $\sim 1 \%$ of the LWR discharged nuclear fuel. Argonne National Laboratory (ANL) has performed a study to define the average composition of the LWRdischarged SNF after 25 years of cooling [2]. One SNF processing step is considered to remove $99.995 \%$ of the uranium and the short-term fission products. The heavy metal from this one processing step consists of $\sim 84.5 \%$ plutonium, $\sim 5.0 \%$ neptunium, $\sim 9.9 \%$ americium, $\sim 0.1 \%$ Curium, and $\sim 0.5 \%$ uranium.

This report describes an accelerator-driven subcritical (ADS) system that can burn the minor actinides (MAs) inventory and a fraction of the plutonium inventory of the discharged SNF and transmute some of the long-lived fission products (LLFPS). The objective of this study is to develop an ADS system that utilize the 131 tons of MAs contained within the 80,000 tons of the discharged SNF for power production. In addition, a fraction of the plutonium inventory is utilized at the same time in this ADS system and some LLFPs are transmuted to stable isotopes. The above average fuel compositions from the LWR discharged fuel are used as the initial fuel compositions in the study.

ADS systems have been considered in closed-fuel-cycle studies for disposing of SNF $[3,4]$ because of the potential advantages of using external neutrons from spallation targets, the enhanced safety features of subcritical assemblies, and the flexibility of loading MAs. Most of the previous studies adopted the traditional solid fuel form to utilize the gained experience from operating fission power reactors [5-7]. This approach requires several new technologies, i.e., developing and testing new fuel forms loaded with MAs, modifying or replacing the current fuel reprocessing techniques to accommodate the new fuel forms, and using several fuel reprocessing steps to recover unused fuel materials. All these developments and the required tests are time-consuming and add significant extra costs. The studies in this report focus on examining an ADS system utilizing mobile fuel forms. The use of mobile fuel eliminates the necessity of developing new solid fuel forms, eases the burden of the radiation damage in the materials for deep fuel burnup, and eliminates the extra fuel-reprocessing steps. 
In the mobile fuel concept, the actinides are dissolved, mixed, or suspended in a liquid fuel carrier. The fuel materials can form compounds or exist as suspended microparticles in the liquid fuel carrier. Therefore, batch or continuous feed operation is feasible to stabilize the reactivity of the subcritical assembly and to control the system power. The liquid fuel carrier, based on the past research studies [8, 9], can be either liquid metals, i.e., lead-bismuth eutectic (LBE), or liquid lead; or molten salts, i.e., FLIBE, other fluorides, or chlorides. In this study, LBE is used first because of its excellent neutron characteristics and low melting point. Then, liquid lead is used as the spallation neutron target and fuel carrier to avoid the polonium generation from bismuth, to reduce the cost of the fuel carrier, to lessen the erosion and the corrosion of the ferritic steel structure.

In an ADS system, the spallation neutron target is placed at the center of the system to maximize its utilizations. It is surrounded by a cylindrical fission blanket. In preliminary studies, graphite was used as the reflector material for the fission blanket, as shown in Figure 1. Steady-state Monte Carlo simulations were performed to define the main parameters of the fission blanket configuration. Monte Carlo fuel burnup analyses were performed to examine the effectiveness of the system for transmuting the MAs.

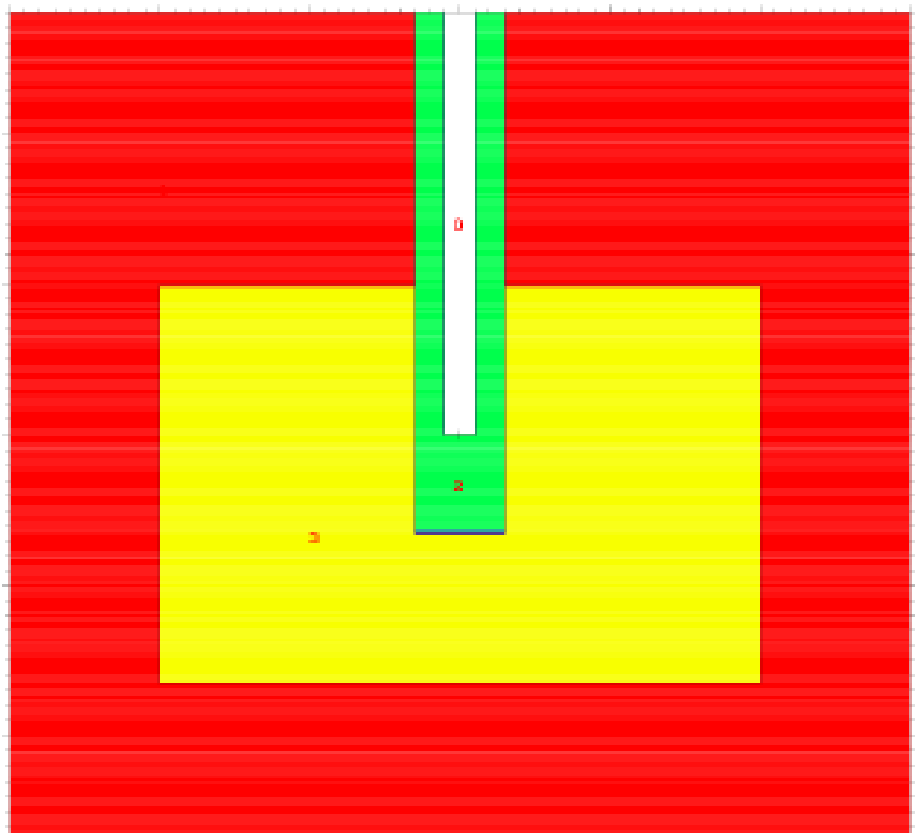

Figure 1. ADS system model (White: proton beam channel; Green: LBE target/coolant material; Blue: HT-9 wall; Yellow: LBE fuel blanket; Red: graphite)

The preliminary concept assumes a homogeneous fission blanket filled with the mobile fuel slurry. The fission power is removed by circulating the liquid mobile fuel outside the subcritical assembly to external heat exchangers. For the later conceptual configurations, steel is used to replace the graphite reflector for eliminating the thermal power peak at the blanket reflector interface caused by the reflected low energy neutrons. The required operating conditions for a satisfactory HT-9 ferritic steel structure performance determine the main design criteria for the thermal hydraulics design 
analyses. The maximum surface temperature of the steel structure material is limited to less than $600{ }^{\circ} \mathrm{C}$ and the maximum liquid mobile fuel velocity is restricted to less than 2 $\mathrm{m} / \mathrm{s}$ for LBE and less than $3 \mathrm{~m} / \mathrm{s}$ for liquid lead [10]. The reactor physics and the thermal hydraulics analyses were coupled to define the ADS concept. The reactor physics calculations used the Monte Carlo codes MCNPX [11], MCB5 [12], and SERPENT [13]; and the thermal hydraulics calculations utilized the commercial computational fluid dynamics (CFD) code STAR-CCM+ [14].

Three concepts with different configurations were considered for the subcritical assembly. The first concept is a homogeneous self-cooled system as mentioned above. The mobile fuel slurry is the primary coolant for removing the generated heat from the fission blanket to the secondary loop outside. The heat exchangers coupling the primary and secondary loops are located outside the vessel of the subcritical assembly. The design analyses show that this concept needs a large fuel inventory circulating outside the subcritical assembly and it is not possible because of the limited amount of MAs [1] in the SNF inventory. The second concept has a separate primary cooling system to remove the generated heat from the fission blanket. In this case, only a small stream of the mobile fuel is slowly circulated outside the fission blanket to remove the short-lived fission products, and to feed the fission blanket with MAs. Two fission blankets have been considered with different coolant arrangement inside the fission blanket. This second concept has the coolant material inside a bundle of tubes inserted inside the fission blanket containing the mobile fuel (referred to as inverted bundle model). In the third concept, the coolant, and the mobile fuel materials of the second concept are inverted, i.e. the mobile fuel carrier is inside a bundle of tubes (referred to as bundle model). For both concepts, reactor physics analyses were performed to determine the fuel compositions, the subcritical assembly size, and the power distributions in the fission blanket. Then, thermal-hydraulic analyses were performed to determine the parameters of the fission blanket relative to the design requirements. The analyses were iterated to satisfy the design requirements and achieve the required performance.

This report discusses the performed analyses for the three concepts. The performed physics analysis to determine the main parameters of the spallation target and the homogeneous fission blanket of the preliminary concept are discussed in section 2 . The fuel burnup results performed for the homogeneous configuration are presented in section 3. Then the physics and the thermal-hydraulic analyses of the second and third concepts are discussed in section 4 to determine their parameters. The fuel burnup analyses of the selected third concept obtained from Section 4 analyses are presented in Section 5. Finally, a summary is given in section 6 .

\section{Physics analysis of the Homogeneous Configuration}

\subsection{Spallation Neutron Source Configuration}

The spallation target generates the neutron source to drive the subcritical assembly and to serve as the interface between the accelerator and the subcritical fission blanket. 
In the spallation reaction, a light projectile (proton for ADS system) with the kinetic energy in the range of several hundred $\mathrm{MeV}$ to several $\mathrm{GeV}$ interacts with a heavy nucleus (ADS target material LBE or lead) and releases a large number of neutrons and other spallation fragments. For the LBE neutron target, the number of generated neutrons normalized to the beam energy saturates as the proton energy exceeds $1 \mathrm{GeV}$ [15]. Based on the Monte Carlo simulation results shown in Figure 2, the 1-GeV protons are stopped after travelling $\sim 62 \mathrm{~cm}$ into the LBE target and deposit $99.8 \%$ of its energy. The $2 \mathrm{GeV}$ protons do not stop before $147 \mathrm{~cm}$. Therefore, the neutron target length for the $1 \mathrm{GeV}$ proton beam is selected to be $\sim 70 \mathrm{~cm}$.

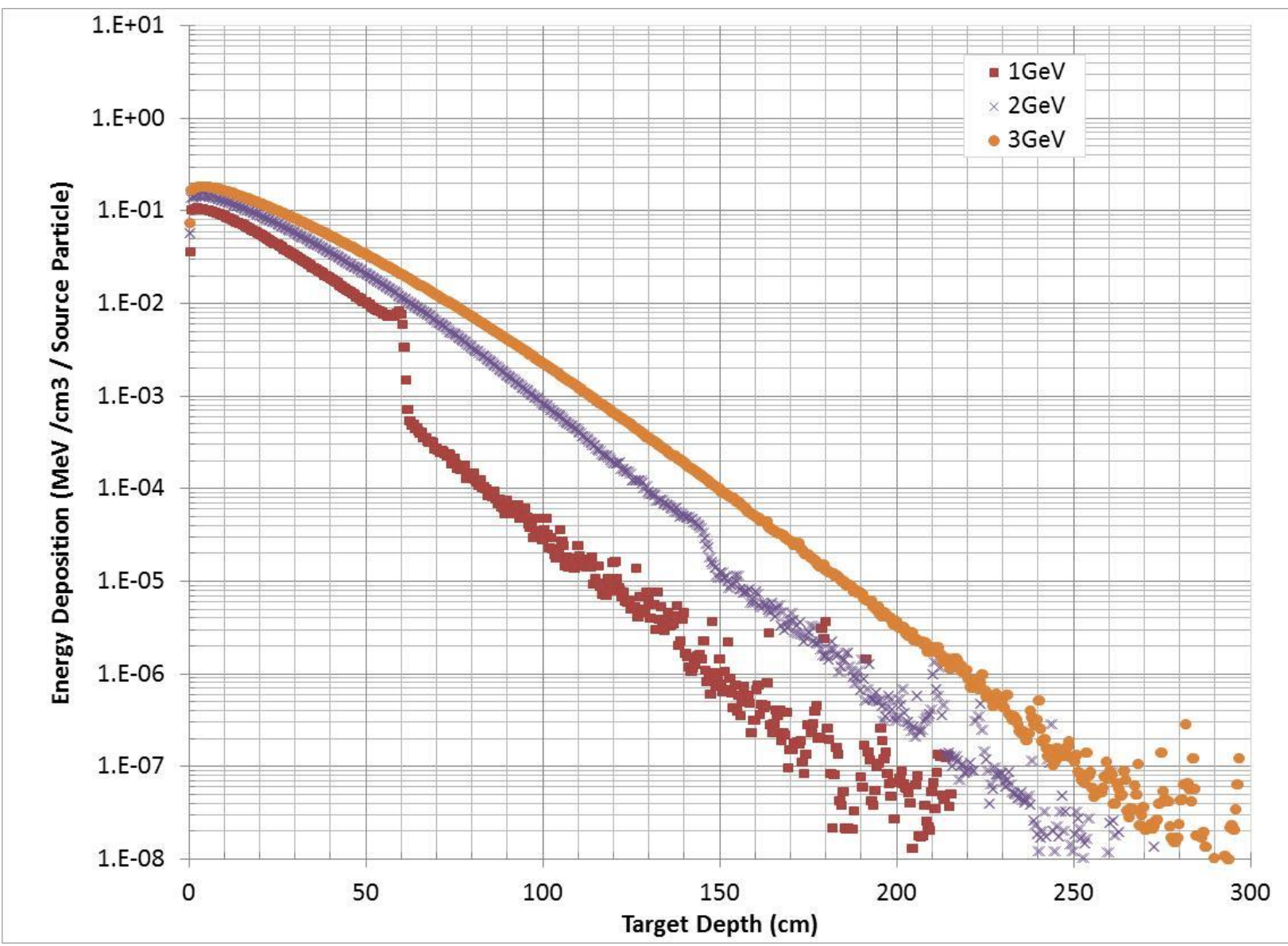

Figure 2. LBE total energy deposition as function of target length for various proton energies

A detailed target design that utilizes LBE as the target material was performed at ANL in the early 2000s [10]. In this design, physics, thermal-hydraulic, radiation effects, and structure analyses have been iterated to satisfy the required engineering constraints. The $24-\mathrm{KW} / \mathrm{cm}^{2}$ heat load for the target window results in a satisfactory target design and an acceptable operating lifetime. An average velocity limit of $2 \mathrm{~m} / \mathrm{s}$ is imposed on the LBE coolant at the structure interface to minimize erosion and corrosion of the structural materials. 
The above limits are adopted for the spallation neutron target of the current ADS systems using the LBE target material. The target geometry was scaled up such that the energy deposition limit is not exceeded. However, the use of $1-\mathrm{GeV}$ protons results in lower power density in the beam window, which provides extra design margin relative to the case with $600-\mathrm{MeV}$ protons as shown in Table I. The key parameters of the $25 \mathrm{MW}$ target design with $1 \mathrm{GeV}$ protons compared with the earlier $5 \mathrm{MW}$ ANL target design with $600 \mathrm{MeV}$ protons are listed in Table I. The target design has a cylindrical geometry with $70 \mathrm{~cm}$ diameter and $70 \mathrm{~cm}$ height.

Table I. Main parameters of the LBE target designs.

\begin{tabular}{|l|c|c|}
\hline \multicolumn{1}{|c|}{ Parameters } & Old ANL Design & New Design \\
\hline Proton Beam Energy, MeV & 600 & 1000 \\
\hline Proton Beam Power, MW & 5 & 25 \\
\hline Window Beam Power, KW/cm ${ }^{2}$ & 24.0 & 24.0 \\
\hline Window Heat deposition. W/cm ${ }^{3}$ & 766 & 323 \\
\hline Proton Beam Radius (with halo current), cm & 10.0 & 19.5 \\
\hline LBE Coolant Outlet Flow Channel Radius, cm & 13.2 & 27.43 \\
\hline LBE Coolant Inlet Flow Channel Radius, cm & 16.5 & 33.13 \\
\hline Steel Outer Wall Radius, cm & 17.0 & 34.27 \\
\hline Target Length, cm & 35.0 & 70.0 \\
\hline Target Radius, cm & 16.5 & 33.13 \\
\hline
\end{tabular}

\subsection{Subcritical Fission Blanket Design}

In the preliminary analyses, the subcritical assembly has a cylindrical fission blanket that surrounds the neutron target. The fission blanket consists of a homogeneous zone with actinide fuel materials dissolved or fuel particles suspended in the LBE fuel carrier. The actinide fuel composition consists of the LWR discharged fuel with 25 years cooling time and $99.995 \%$ of the uranium and the short-lived fission products are removed. It has the following composition: 84.5\% plutonium (1.27\% Pu238, 53.20\% Pu239 21.53\% Pu240, 3.78\% Pu241, and 4.69\% Pu242), 5.02\% Np237, 9.9\% Am (8.97\% Am241, $0.014 \% \mathrm{Am} 242 \mathrm{~m}$, and $0.93 \% \mathrm{Am} 243), 0.11 \% \mathrm{Cm}$, and $0.5 \% \cup[2]$. In this study, the actinides are divided into two parts: the first part has the plutonium and the leftover uranium; and the second part has the MAs; Np, Am, and Cm elements. Since, the main goal is to burn the MAs, the fuel compositions minimized the first part, which has the plutonium as the main component. The main isotopes of the MAs, Np-237 and Am-241, are fertile isotopes in the thermal neutron spectrum and they have small fission crosssection values in the fast neutron spectrum. Therefore, some plutonium is needed to enhance the neutron multiplication of the subcritical fission blanket. The actinide fuel used in this analysis is composed of the two parts, with the relative isotope concentrations in 
each part are kept as it is obtained from the LWR discharged fuels but the relative concentrations between the two parts adjusted to load more MAs as possible. No isotopic separation or enrichment is considered in this study. Only one LWR SNF processing step was performed to remove the uranium and the short-lived fission products.

The neutron multiplication of the fission blanket depends on the added Pu fraction in the fuel material and the volume fraction of the loaded fuel material in the liquid fuel carrier. The solubility of the actinide materials in the LBE is low and it is less than $2 \%$ at $900 \mathrm{~K}$ [16-17]. Experimental work by Hahn [8] showed that LBE with $13 \%$ reduced uranium oxide particles concentration forms a stable slurry. First, numerical simulations were performed to study the neutron multiplication factor as a function of the plutonium concentration in the actinide fuel material and the fuel particles volume fraction loaded in the LBE slurry.

Figure 3 shows the infinite neutron multiplication factors of fission blankets with different actinide fuel volume fractions and Pu concentrations in the actinide fuel material. To achieve a neutron multiplication factor of 0.98 , there is a minimum Pu concentration for each fuel fraction loaded in the LBE, i.e., $~ 40$ at $\%$ Pu for the $2 \%$ actinides, $\sim 13$ at $\% \mathrm{Pu}$ for the $10 \%$ actinides loaded in LBE.

For an ADS system, the fission power generated in the blanket is proportional to the accelerator beam power and the neutron multiplications. Figure 4 shows the corresponding total ADS power using 25-MW beam power delivered with $1-\mathrm{GeV}$ protons as a function of the plutonium concentration in the actinide fuel material assuming $7 \%$ actinides loaded in the LBE fuel carrier, and the fission blanket effective neutron multiplication factor $k_{\text {eff. }}$ Based on the results of Figure 4, the neutron multiplication factor of the fission blanket should be $\sim 0.98$ to generate $3 \mathrm{GW}$ thermal power using $25 \mathrm{MW}$ beam power delivered with $1 \mathrm{GeV}$ protons.

The total MA inventory and the fission blanket volume are shown in Figure 5 as a function of the plutonium concentration in the actinide material and different actinide material concentrations in the LBE fuel carrier for blankets. The effective neutron multiplication factors of these blankets are 0.98. The initial MA inventory loaded in the fission blanket is constrained by the SNF processing capacity and the time difference between the start of the SNF processing and the first ADS system operation. If the 80,000 MTHM SNF can be processed within 40 years, about 13 tons of MAs will be available within 4 years to start the first ADS system. Figure 5 can be used to define the desired blanket configuration as a function of the MA inventory and the desired actinide concentration in the LBE fuel carrier. Three possible blanket configurations are listed in Table II. Each configuration has a different plutonium fraction in the fuel material and a different fuel material loading in the slurry. In these blanket configurations, the total fuel carrier volumes are about or less than $20 \mathrm{~m}^{3}$ and the amount of required MAs inventories are about or less than 10 tons. 


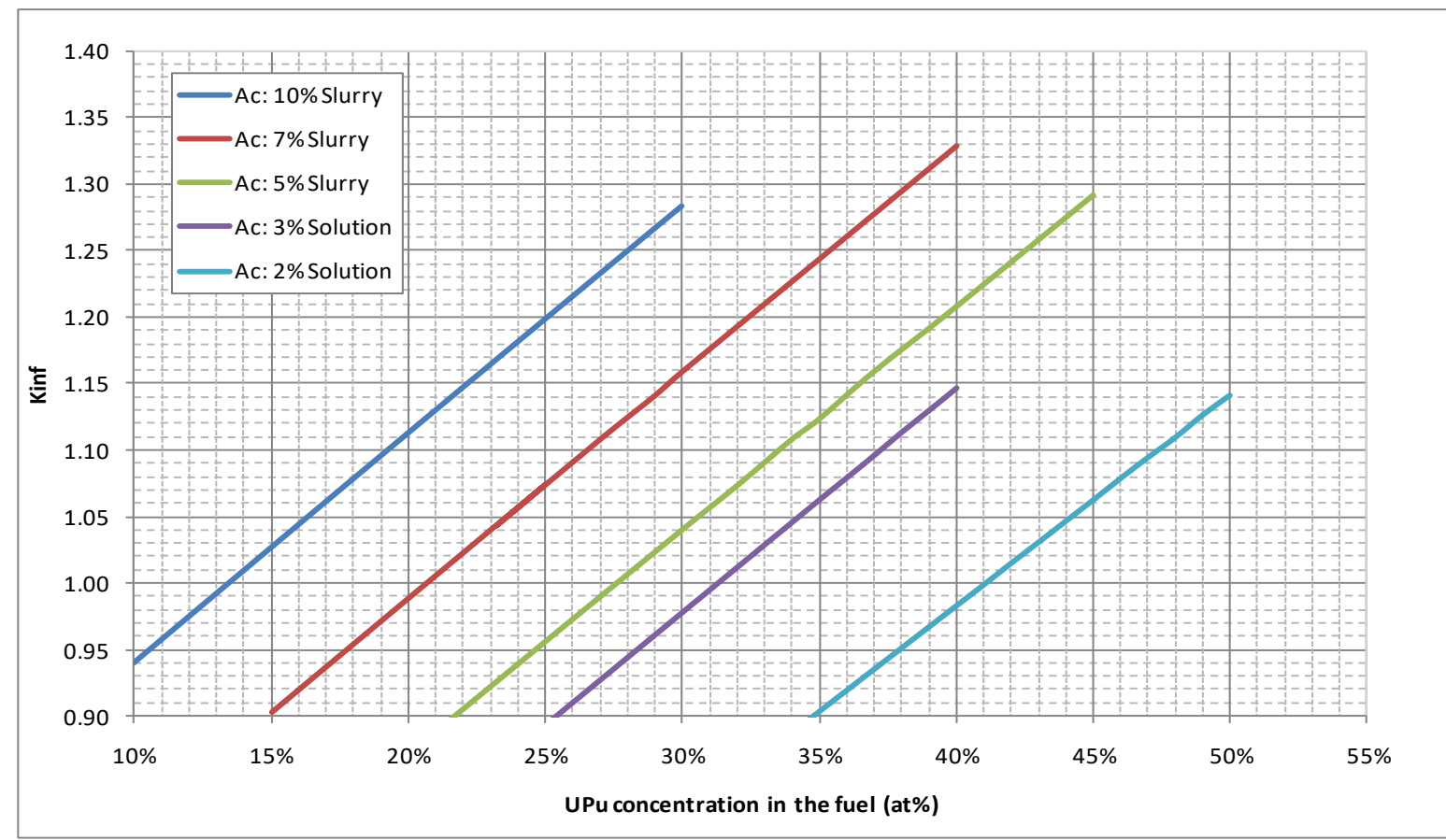

Figure 3. The fission blanket infinite neutron multiplication as a function the U-Pu concentration in the actinide material for different actinide concentrations in the LBE fuel carrier

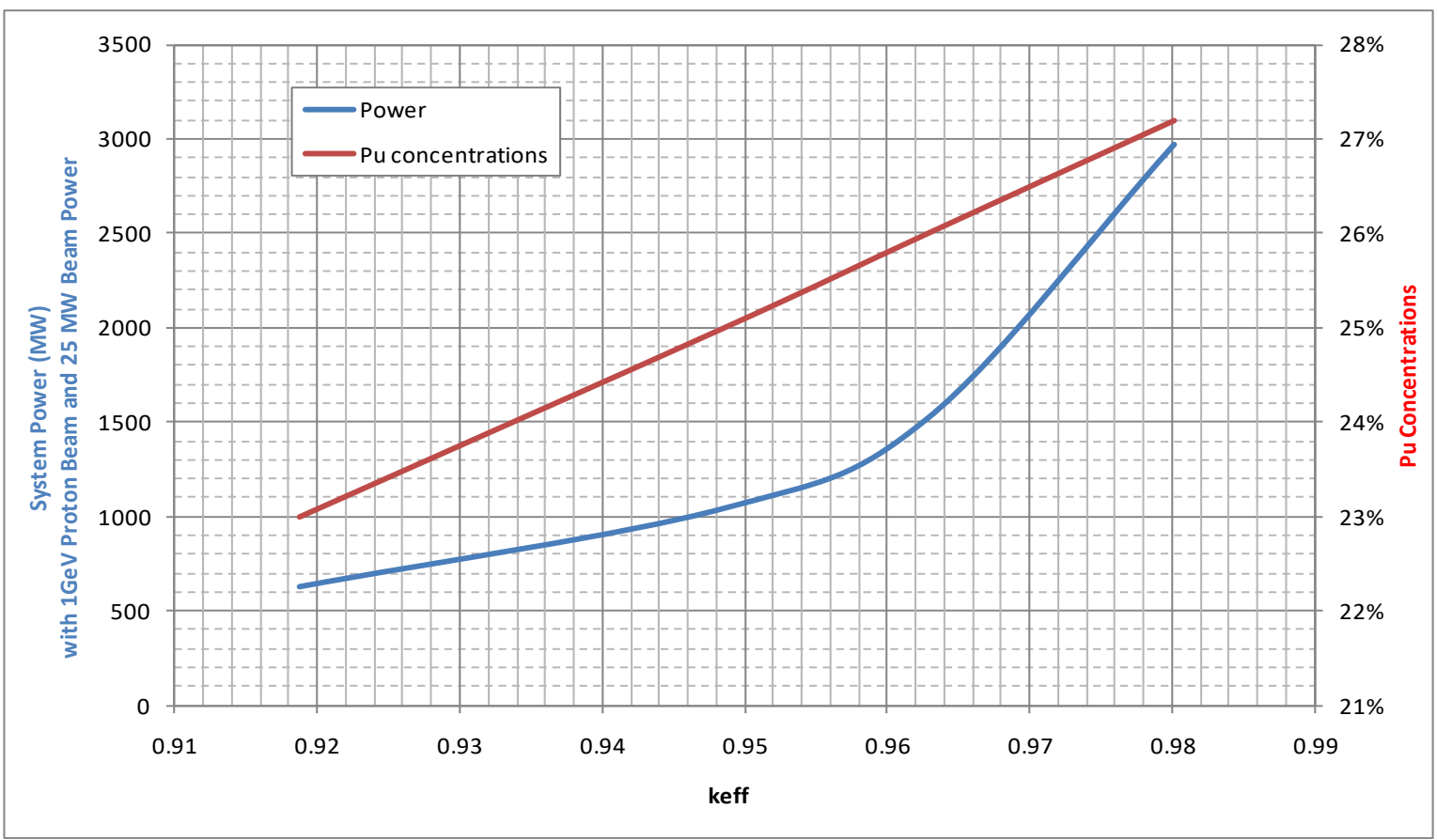

Figure 4. The fission blanket effective neutron multiplication as a function of the plutonium concentration in the actinide fuel material with $7 \%$ actinides loaded in the LBE fuel carrier, and the corresponding total ADS power using $25 \mathrm{MW}$ beam power delivered by $1 \mathrm{GeV}$ protons as function of the $\mathrm{k}_{\text {eff }}$ value 


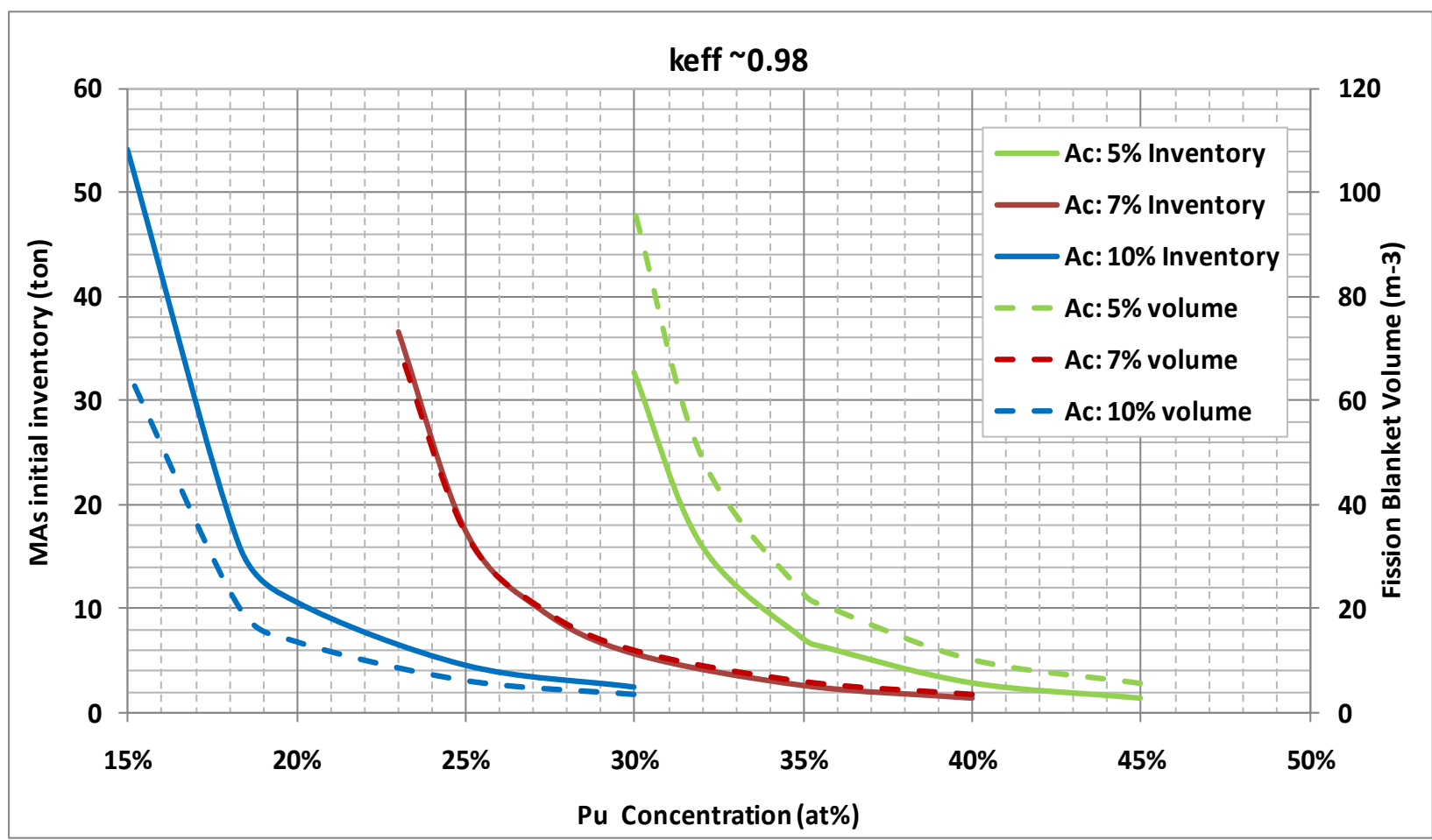

Figure 5. The total MA inventory and the fission blanket volume as a function of the plutonium concentration in the minor actinide material for different actinide material concentrations in the LBE fuel carrier, all blankets with an effective neutron multiplication factor of 0.98

Table II. Three fission blanket configurations with $0.98 \mathrm{k}_{\text {eff }}$ and MA inventory $\leq 10$ tons.

\begin{tabular}{|c|c|c|c|c|c|}
\hline Keff & $\begin{array}{c}\text { Actinide } \\
\text { loading }\end{array}$ & $\begin{array}{c}\text { Plutonium } \\
\text { loading }\end{array}$ & $\begin{array}{c}\text { Blanket } \\
\text { Volume, } \\
\mathrm{m}^{3}\end{array}$ & $\begin{array}{c}\text { Height or } \\
\text { Diameter, } \\
\mathrm{m}\end{array}$ & $\begin{array}{c}\text { MA } \\
\text { loading, } \\
\text { ton }\end{array}$ \\
\hline 0.980 & $5 \%$ & $35.7 \%$ & 20.4 & 3.0 & 6.33 \\
\hline 0.980 & $7 \%$ & $27.2 \%$ & 20.4 & 3.0 & 10.0 \\
\hline 0.980 & $10 \%$ & $20.0 \%$ & 13.7 & 2.64 & 10.6 \\
\hline
\end{tabular}

\section{Preliminary Fuel Burnup Analysis of the ADS System}

In subcritical blankets, the actinide consumption rate is determined by the fission power generated. During the operation of the ADS system, the neutron multiplication factor of the blanket decreases as the fissile materials concentration decreases. However, a high concentration of Am-241 isotope in the mobile fuel affects the effective neutron multiplication factor of the blanket. In the fuel burnup process, the Am-241 atoms are fissioned, or transmuted to the fissile isotopes Am-242g or Am-242m through neutron capture, as shown in the diagram below. The various reaction rates shown in the diagram 
were calculated for a fission blanket configuration with $0.98 \mathrm{k}_{\text {eff }}$ and $3 \mathrm{GW}$ thermal power output.

Apparently, the majority of the Am-242g produced from the Am-241 neutron capture decays quickly to the fertile isotopes Pu-242 or Cm-242. At the same time, the Am-242m has a relatively long half-life of 141 years. Therefore, the Am-242m isotope will be consumed mainly by fission. A small fraction of the Am-242m isotope is transmuted to Am-243 by neutron capture or decayed to Am-242g. Due to the large concentration of Am-241 in the actinide fuel material, the Am-242m concentration increases during the first few years of operation. The Am-242m concentration depends on the branching ratio value of Am-241 neutron capture to Am-242m. Depending on the generated amount of Am$242 \mathrm{~m}$, the neutron multiplication factor is decreased or increased in the first few years of operation.

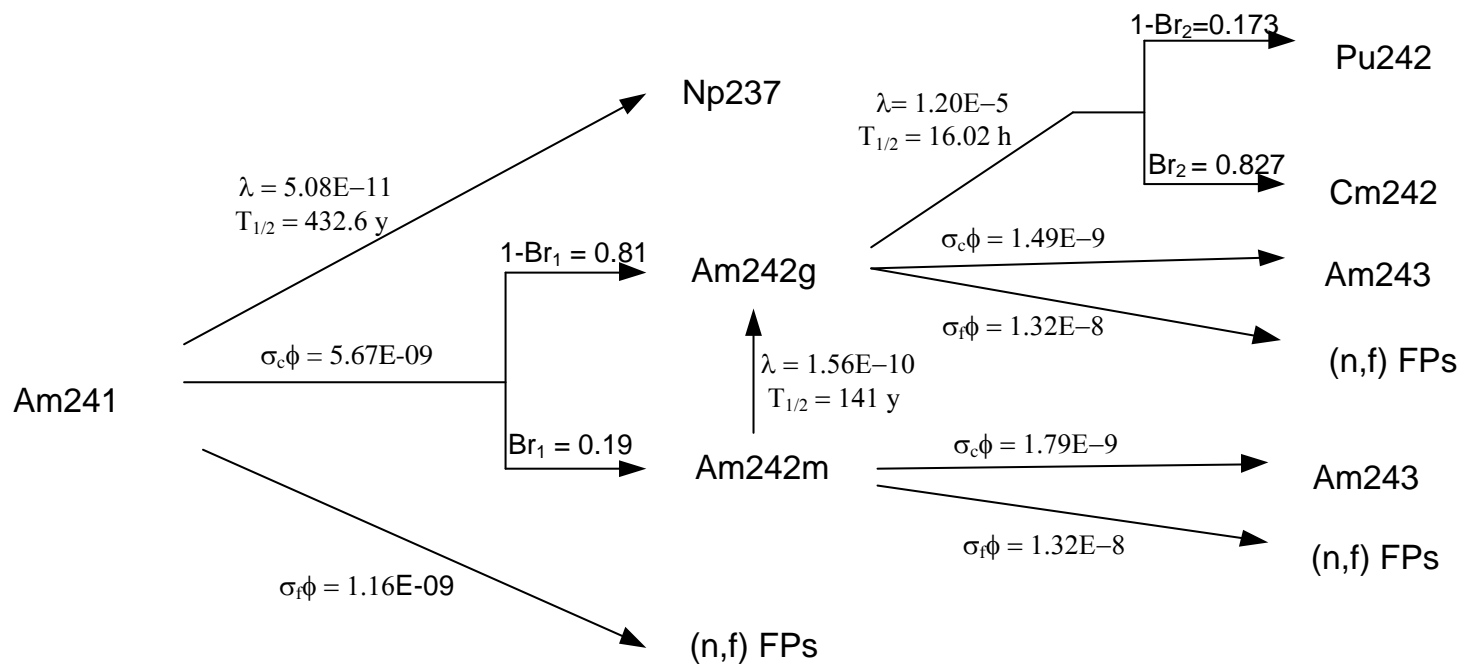

$\mathrm{Br}_{2}$ branching ratio in the above diagram has a fixed value and the branching ratio $\mathrm{Br}_{1}$ depends on the neutron energy. $\mathrm{Br}_{1}$ has limited number of the experimental data points and the nuclear data libraries show significant differences regarding its value [18]. The ENDF/B-VII nuclear data files have improved this branching ratio based on new differential data and integral measurements [19]. The MCB5 Monte Carlo burnup code [12] utilizes an old branching ratio values calculated by Mann and Schenter [20]. In MCNPX, this branching ratio is calculated based on CINDER library. As shown in Figure 6 , these branching ratio values are significantly different from one another at various neutron energy. In this report, the Monte Carlo burnup simulations performed with the MCB5 code use the Am-241 branching ratios data of ENDF/B-VII nuclear data files.

For the burnup analyses, a Monte Carlo simulation was first performed to obtain the spallation neutron source from the LBE target, utilizing the surface source function of MCNPX without the impact of the fission blanket. In the Monte Carlo model, all zones outside the LBE target except the target assembly are voided. Neutron leakage from the LBE target through the target surfaces was recorded, including all the characteristic 
parameters of each neutron. Energy, position, and direction of each neutron are saved in the SSRA source file.

In the preliminary analysis, the Monte Carlo burnup simulations were performed to evaluate the performance of the three ADS blanket configurations listed in Table II. The MCB5 code used the generated SSRA neutron source file. All regions in the actual ADS conceptual configuration as well as the target region were loaded with the actual materials. The system thermal power was fixed at $3 \mathrm{GW}$, which is used to normalize neutron flux during the burnup simulations. The fuel burnup time-step is set to 90 days.

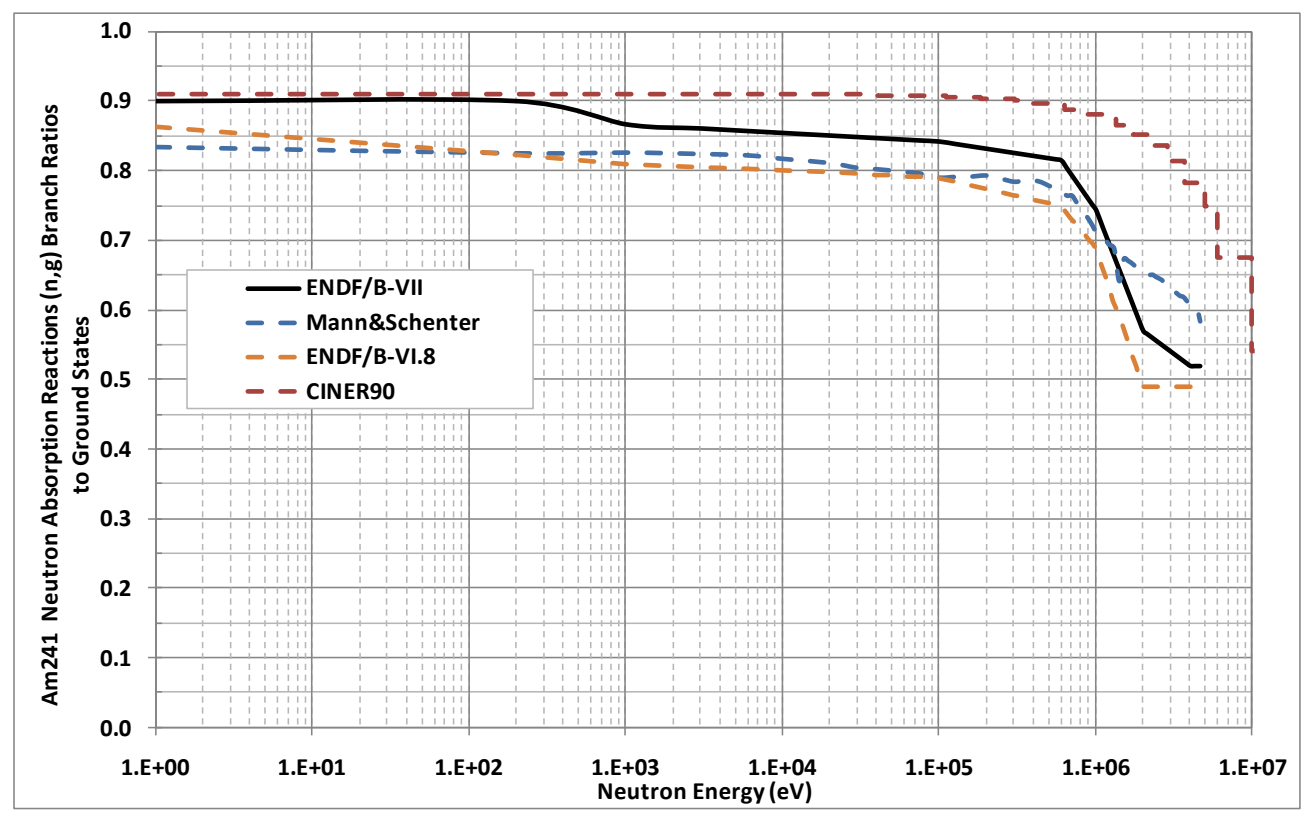

Figure 6. Am-241 $(\mathrm{n}, \mathrm{g}) \mathrm{Am}-242 \mathrm{~g}$ branching ratios from Mann \& Schneter calculation, ENDF/B-VI.8, -VII.0 nuclear data files, and CINDER90 library.

During the fuel burnup, the blanket neutron multiplication factor increases due to the Am-242m buildup or decreases due to the fissile fuel consumption. To control the $k_{\text {eff }}$ value of the fission blanket at $\sim 0.98$ and consequently the output thermal power, continuous or batch feeding process is required to add actinides, to remove short-lived fission products, or to add long-lived fission products. In this analysis, batch feeding at the end of each time step was utilized to control the $k_{\text {eff }}$ value. Neutron absorbers are fed into the system when $k_{\text {eff }}$ increases. Two long-lived fission products, Tc-99 and I-129, were added to the fission blankets to decrease the $k_{\text {eff }}$ value. Actinide materials were added to the blanket to compensate for the reactivity loss when $k_{\text {eff }}$ decreases. The added actinide materials are the same as the initial actinide materials in the LBE fuel carrier. The amount of feeding materials, neutron absorbers, or actinide materials was calculated so that the $\mathrm{k}_{\text {eff }}$ value of the fission blanket after the feeding process returns to the initial value of $\sim 0.98$ [21]: 


$$
f_{j}=\frac{\left(\frac{1}{k_{1}}-\frac{1}{k_{0}}\right) \sum_{i} \chi_{i} v \sigma_{f, i} N_{i, 1} \phi_{1}}{\frac{1}{k_{0}} \sum_{k} \chi_{k} v \sigma_{f, k} N_{k} \phi_{1}-\sum_{k} \sigma_{\gamma, k} N_{k} \phi_{1}}
$$

Where, $f_{j}$ is the amount of feed material fraction calculated for the $j^{\text {th }}$ burnup step. It is defined as the fraction of the feeding vector $N$ with atom density $N_{k}$ for each feeding isotope component. $k_{0}$ and $k_{1}$ are the $k_{\text {eff }}$ values at the beginning and end of the $j^{\text {th }}$ time step respectively. $\phi_{1}$ is the neutron flux calculated at the end of the $j^{\text {th }}$ time step. $\sum_{i} \chi_{i} v \sigma_{f, i} N_{i, 1} \phi_{1}$ is the total neutron production rate from all the isotopes in the subcritical assembly at the end of the $j^{\text {th }}$ time step. $\sum_{k} \chi_{k} v \sigma_{f, k} N_{k} \phi_{1}$ is the total neutron production rate from all the isotopes in the feeding vector using fluxes at the end of the $j^{\text {th }}$ time step. $\sum_{k} \sigma_{\gamma, k} N_{k} \phi_{1}$ is the calculated total neutron capture rate of all the isotopes in the feeding vector using the fluxes at the end of the $\mathrm{j}^{\text {th }}$ time step. If $f_{j}$ has a positive value, MAs are loaded for the $j^{\text {th }}{ }^{\text {b }}$ burnup step. If $f_{j}$ has a negative value, LLFPs are loaded for the $j^{\text {tht }}$ burnup step.

Figure 7 shows the calculated $k_{\text {eff }}$ at each time step before and after the batch feeding for the three blanket configurations. The $k_{\text {eff }}$ values have been maintained around 0.98 at the end of each time-step by adding actinides or LLFPs as needed. For the blanket configuration with $5 \%$ actinide concentration, $\mathrm{k}_{\text {eff }}$ always decreases for all the fuel burnup steps. The other two blanket configurations with $7 \%$ and $10 \%$ actinide concentrations, $\mathrm{k}_{\text {eff }}$ increases in the first 2 years because of the Am-242m accumulation and decreases later.

Figures 8 through 10 show the amount of added materials at each time-step for each blanket configuration to maintain $\sim 0.98 \mathrm{k}_{\text {eff }}$ value. As shown in Figure 8 , the annual amount of LLFPs fed into the system is always zero for the configuration with $5 \%$ actinide concentration. The annual amount of actinide feed in this configuration increases gradually in the first 6 years of fuel burnup, and then reaches a constant value of about 1.5 ton/year. For the configurations with $7 \%$ or $10 \%$ actinide concentrations, as shown Figures 9 and 10, LLFPs were added during the first two years to compensate for the positive reactivity increases. Then actinides are added to compensate for the reactivity losses due to the burnup. The annual amount of actinides fed into the system gradually increases and saturates at a level of about 1.5 tons/year after 8 to 10 years of operation. After 10 years of burnup, the total weights of the LLFPs added for the three configurations are $0,1.18$, and 2.15 tons for the configurations with 5,7 , and $10 \%$ actinide concentration in the LBE, respectively. The corresponding total actinide amounts are 11.7, 9.35, and 9.03 tons, respectively.

For the three blanket configurations, the annual transmutation rates of the total actinides and the MAs are shown in Figure 11. The total transmutation rate of the total actinide fuels is 1.19 tons for the three configurations. The ADS systems are very efficient in transmuting MA fuel in the first few years of burnup. The transmutation rate peaks after about two burnup years and then decreases gradually. The plutonium transmutation rates decreases at the beginning of the fuel burnup because of the production of Pu-238 from 
the MAs. Then the annual transmutation rate of the plutonium increases. Overall, the transmutation rates of the MAs and the plutonium saturate after 10 years for the three configurations. The total amount of actinides transmuted during the first 10 years of operation are $\sim 11.9$ for all three cases, among which the total MAs consumed are 9.85, 11.80 , or 12.68 tons for the configurations with 5,7 , or $10 \%$ actinides in the LBE, respectively. The corresponding annual MAs fuel transmutation rates are $0.83,094$, and 1.02 tons/year for the three configurations, respectively, after reaching equilibrium at 10 burnup years. Therefore, the total MA weights burned by the three configurations are 30.6, 35.3, and 37.2 tons, respectively, assuming that the ADS system can operate for 35 years. To utilize the 131 tons of MAs from the 80,000 tons of SNF, 4.3, 3.7, or 3.5 ADS systems are required respectively. During operation, the fuel carrier will be slowly processed for removing the short-lived fission products. However, in the analyses, the short-lived fission products were not removed. Removing the short-lived fission products in the burnup analyses will improve the fission blanket performance.

\section{ADS Conceptual Configurations}

The physics analyses in the previous section assumed that the subcritical fission blanket is filled with slurry fuel carrier and the $3 \mathrm{GW}$ fission power can be properly removed by circulating the slurry fuel carrier outside the subcritical blanket. This section discusses the conceptual configurations of the ADS system developed with the focus on the integration of the heat removal system and the subcritical fission blanket. The 0.98 neutron multiplication factor of the fission blanket and the $25 \mathrm{~mA} 1 \mathrm{GeV}$ proton beam for generating the spallation neutron source are maintained for the development of these ADS systems.

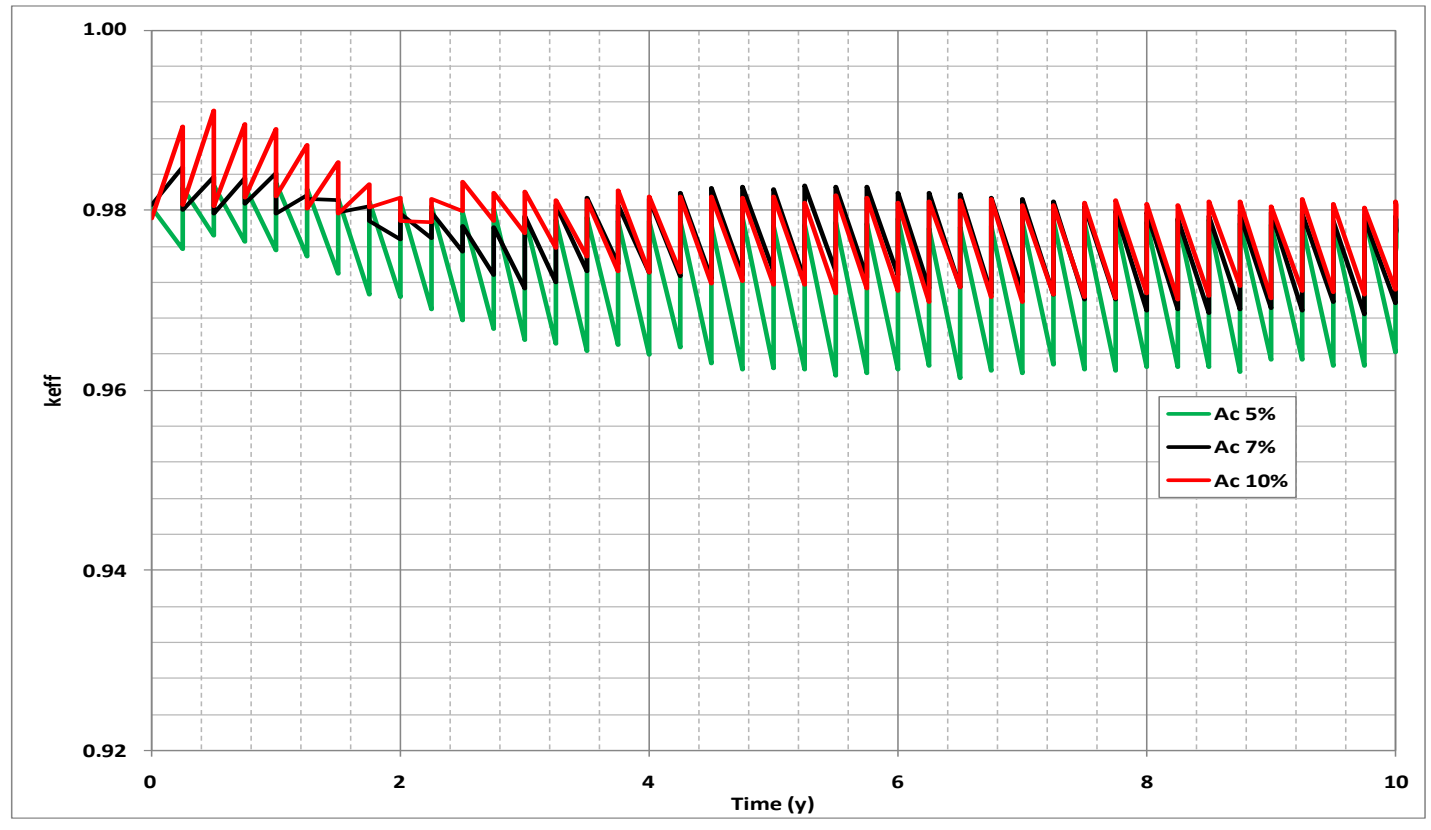

Figure 7. Effective neutron multiplication value before and after each fuel burnup time step for the three fission blanket configurations of Table II 


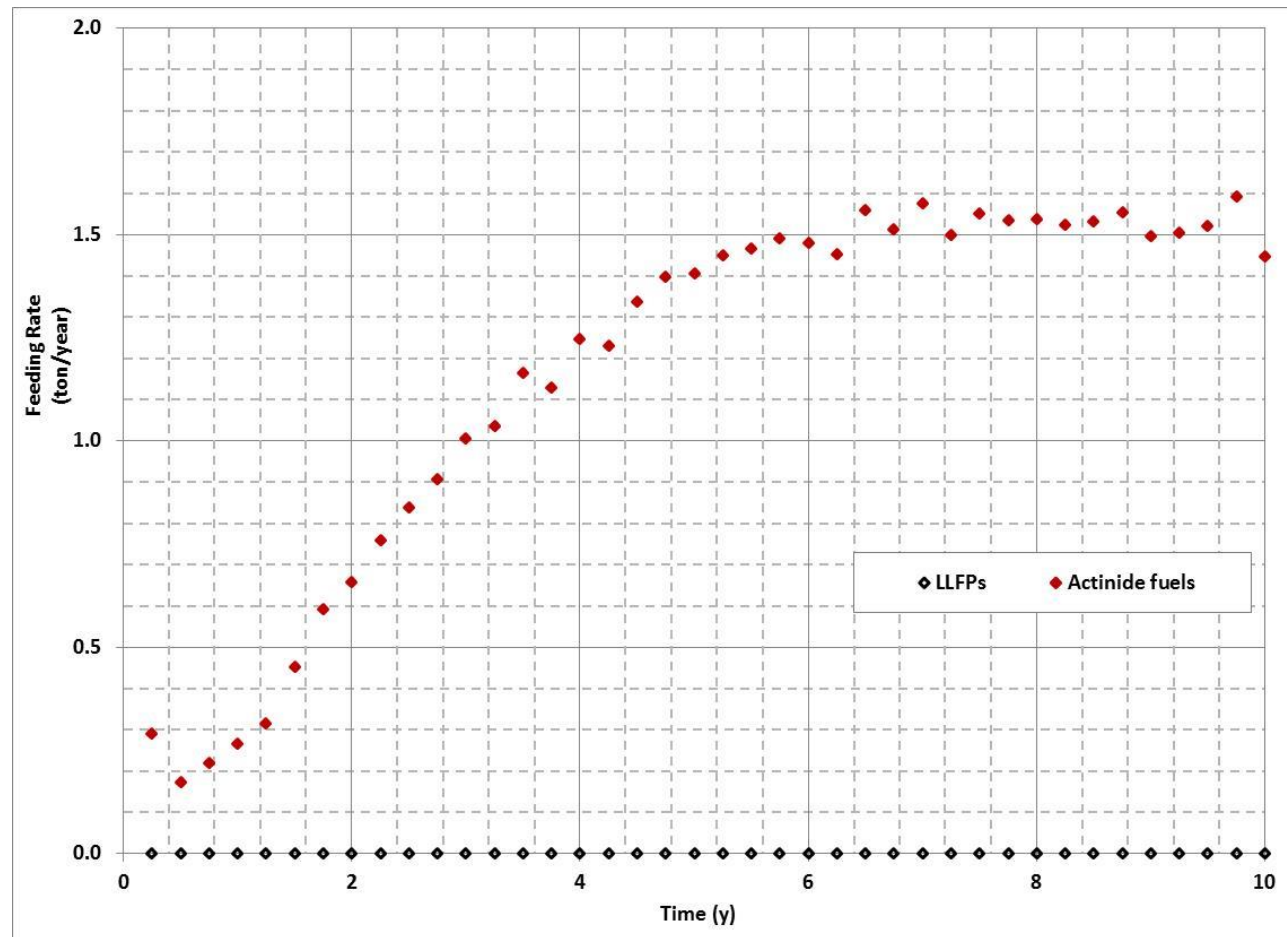

Figure 8. Actinides and LLFPs annual feeding rates at end of each burnup step for blanket configuration with $5 \%$ actinide concentration in LBE and $35.7 \%$ plutonium concentration in actinides

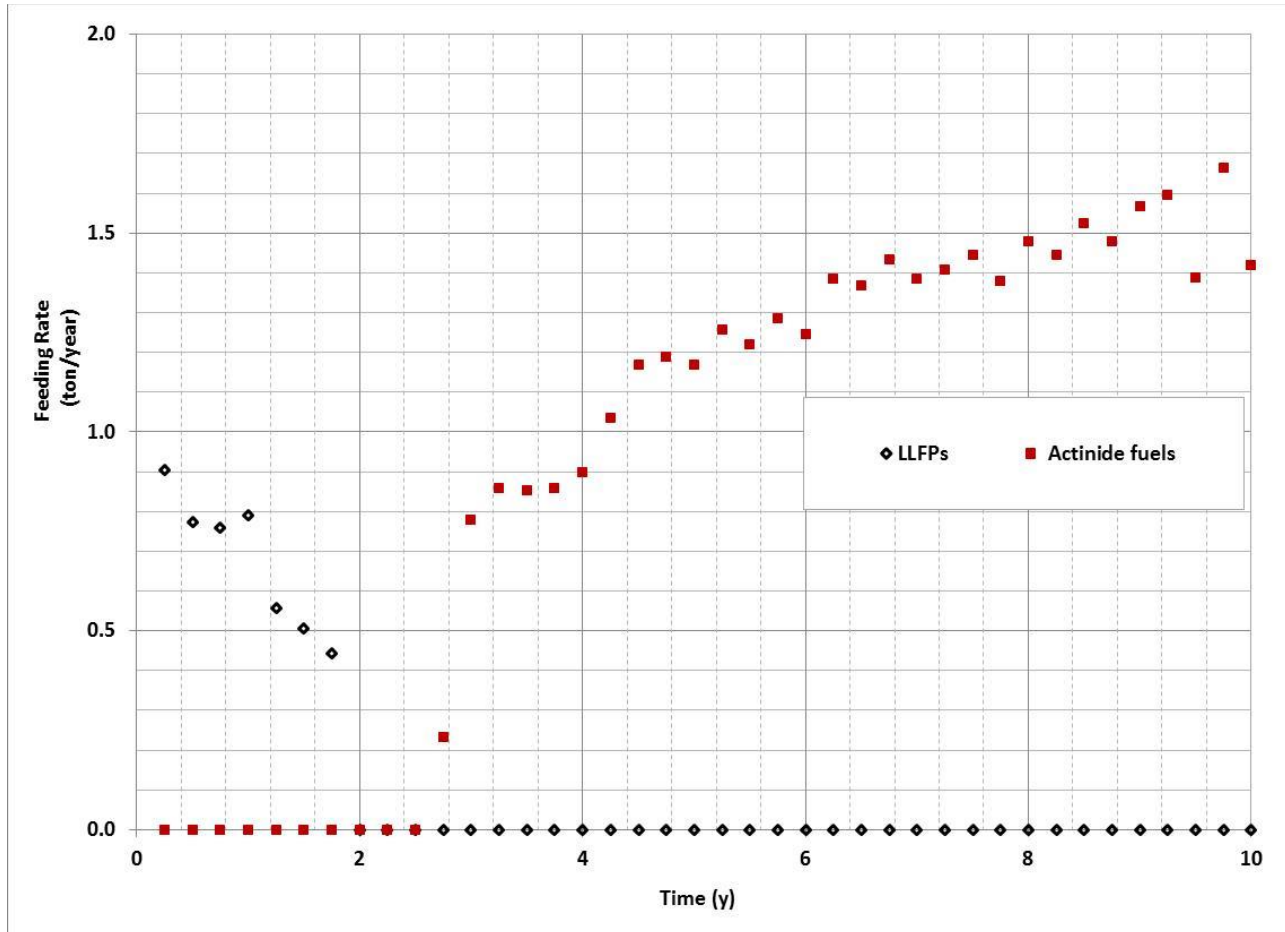

Figure 9. Actinides and LLFPs annual feeding rates at end of each burnup step for the blanket configuration with $7 \%$ actinide concentration in LBE and $27.2 \%$ plutonium concentration in actinides 


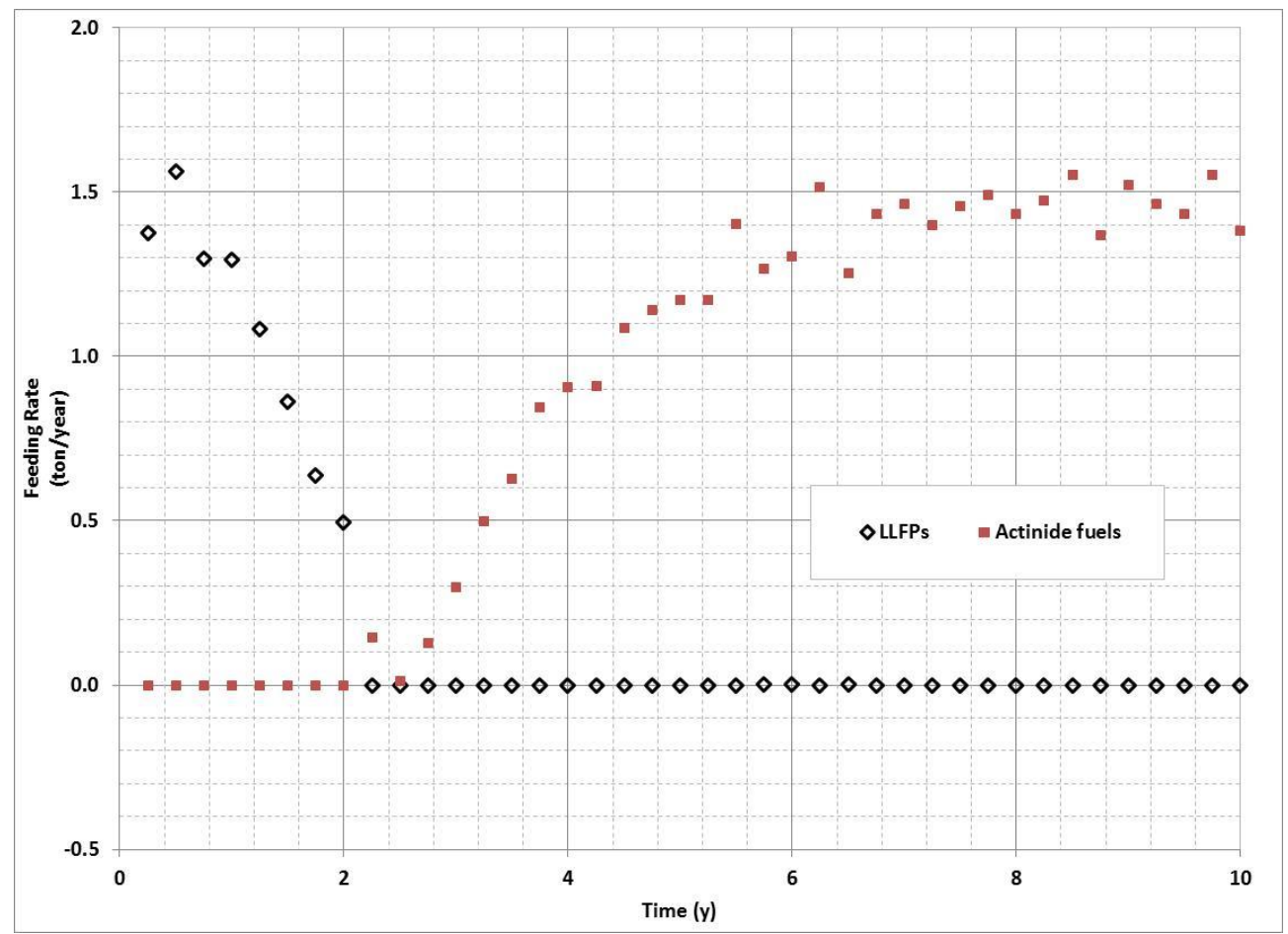

Figure 10. Actinides and LLFPs annual feeding rates at end of each burnup step for the blanket configuration with 10\% actinide concentration in LBE and $20 \%$ plutonium concentration in actinides

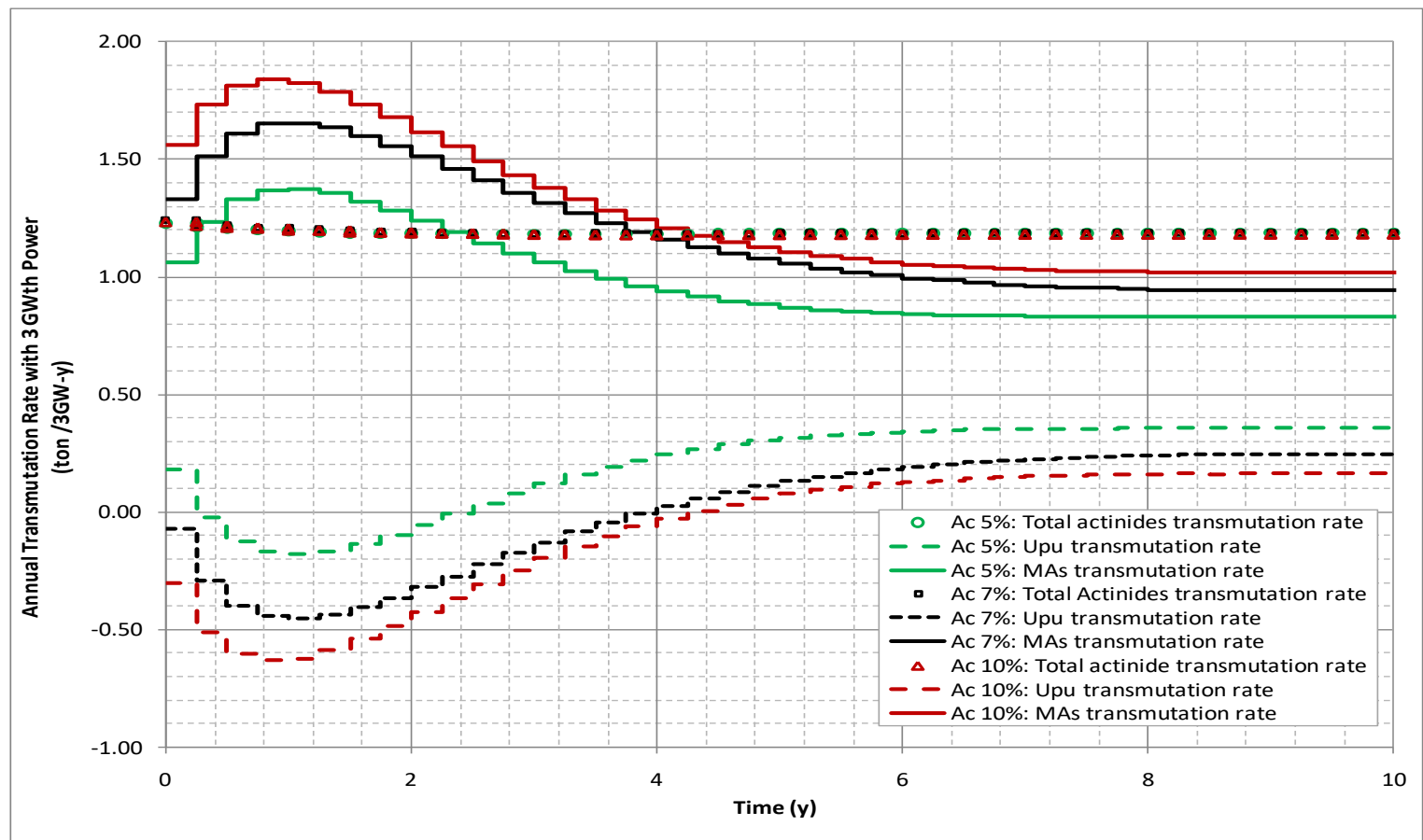

Figure 11. Total actinides, U-Pu, and MAs transmutation rates for three blanket configurations of Table II. 


\subsection{Target Design}

The molten $\mathrm{Pb}$ is used as the spallation neutron target for designing the ADS systems. Lead and LBE have very similar neutron characteristic. For instance, both materials produces about the same number of spallation neutrons per $1 \mathrm{GeV}$ proton, and the stopping distance of the $1 \mathrm{GeV}$ protons in both materials are $\sim 60 \mathrm{~cm}$. The use of lead avoids the generation of Po-210 from bismuth in the system and it is less corrosive than LBE.

A schematic representation of the neutron target with the liquid lead is shown in Figure 12. Table III lists the main geometrical parameters compared with the previous LBE target design. The lead target parameters were slightly adjusted based on the design requirements.

Table III. Main parameters of the neutron target designs using liquid Pb or LBE.

\begin{tabular}{|l|c|c|}
\hline \multicolumn{1}{|c|}{ Neutron Target } & Pb & LBE \\
\hline Proton Beam Energy, MeV & 1000 & 1000 \\
\hline Proton Beam Power, MW & 25 & 25 \\
\hline Proton Beam Radius (with halo current), cm & 20.07 & 19.5 \\
\hline Coolant Outlet Flow Channel Radius, cm & 27.43 & 27.8 \\
\hline Coolant Inlet Flow Channel Radius, cm & 33.13 & 33.8 \\
\hline Steel Outer Wall Radius, cm & 34.27 & 35.0 \\
\hline Target Length, cm & 75.0 & 70.0 \\
\hline Target Radius, cm & 33.13 & 33.8 \\
\hline
\end{tabular}

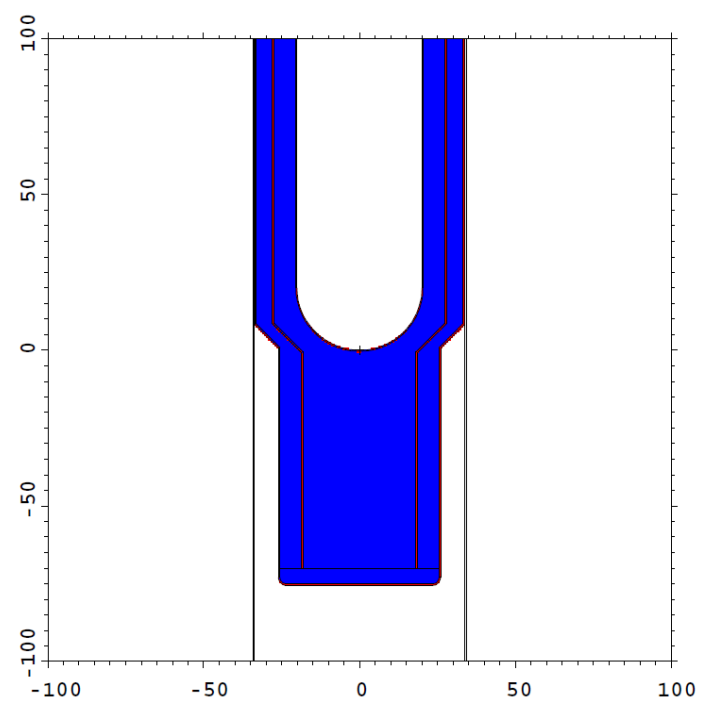

Figure 12 Spallation neutron target model for the ADS system (White: proton beam; Blue: target material; Red: HT-9 structure wall). 
Monte Carlo simulations are performed to calculate the nuclear heat deposition in the liquid lead target with the 25-MW $1-\mathrm{GeV}$ proton beam. Thermal hydraulic analyses have been performed to calculate the thermal hydraulics parameters and the structure temperature distribution of the target design.

The simulated target design is similar to the design analyzed in the previous work [10]. The target has a cylindrical outer shape, with a series of steel shell layers as shown in Figure 13. The innermost tube is the beam tube, which is capped at the bottom by a hemispherical window. The coolant is outside the beam tube and it is separated into "inlet" and "outlet" paths by a separation wall. This wall has a rounded fairing at the bottom. Outside this wall is the outer coolant boundary wall. Outside this boundary is a helium zone that provides an inert space for maintenance and leak protection. The helium zone is bounded by the outer target boundary, which separates the target from the subcritical assembly. The subcritical assembly is centered axially at lowest point of the beam window.

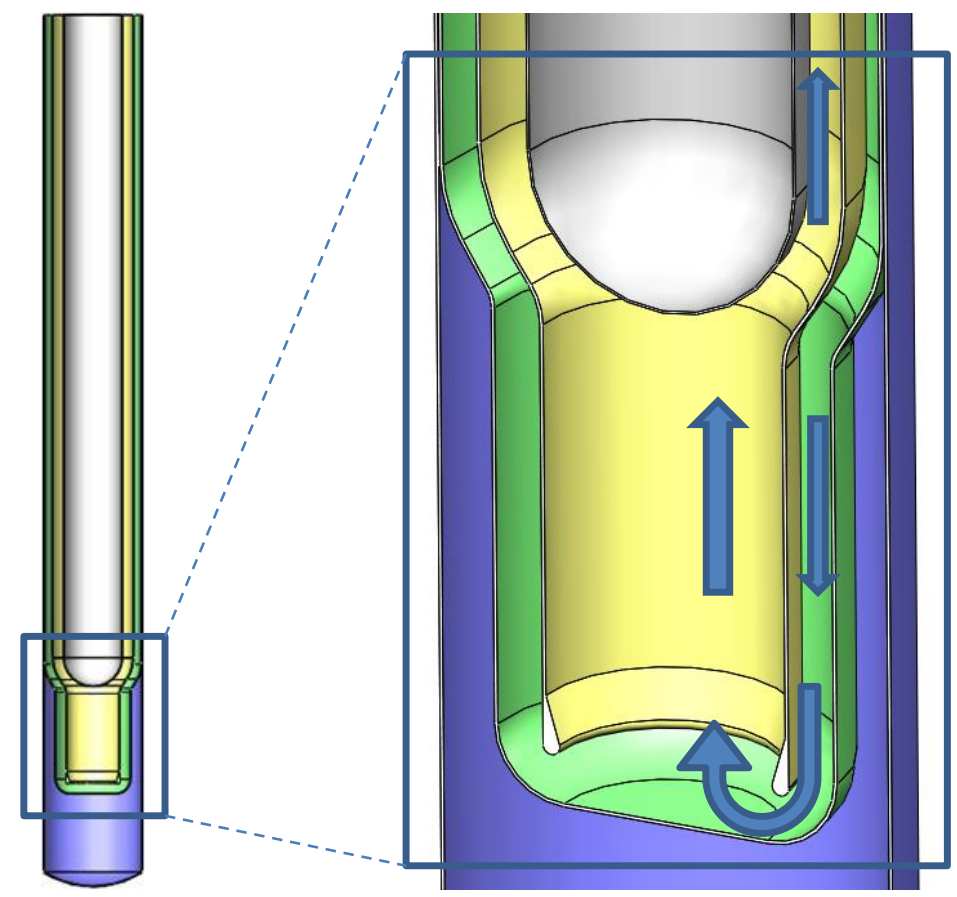

Figure 13 Full (left) and zoomed (right) views of the target assembly, with the coolant flow path illustrated. Gray is the beam tube, yellow is the inlet/outlet separation wall, green is the coolant-bounding wall, and blue is the target guard vessel.

The current target design has a $25 \mathrm{MW}$ beam power, so the target design from the previous work was scaled up. This is a straightforward to do with a simple radial proportionality scaling given the simplicity of the target design. For constant velocity, doubling the target radius quadruples the flow area and hence the mass flow rate. Wall thicknesses remain the same, and the separation wall is placed such that the inlet and outlet coolant channels have equal area, i.e. similar velocities. The beam window is 3.5 $\mathrm{mm}$ thick, while the other walls are $5 \mathrm{~mm}$ thick. 
There are some notable differences for the new target design. First, liquid lead is used instead of LBE. This causes the inlet temperature to be $650 \mathrm{~K}$, which is necessarily higher than that of the LBE case to provide sufficient margin from freezing. This also allows a higher coolant velocity to be used in the target because lead is less corrosive than LBE. For this reason, while most cases used $2 \mathrm{~m} / \mathrm{s}$ for the inlet velocity, $3 \mathrm{~m} / \mathrm{s}$ was also investigated.

The flow path of the coolant is relatively straightforward as shown in Figure 13. The coolant flows downward from the outer coolant channel, and makes a sharp $180^{\circ}$ bend around the bottom of the separation wall. It then proceeds up toward the beam window. The flow leaves the target through the inner channel. The coolant travels for about $3 \mathrm{~m}$ above the beam window, so some heat transfer between the inlet and outlet coolant flows is expected. It is also known that due to the sharp, relatively high-speed bend, flow separation can occur at the bottom of the separation wall. The prediction of this separation is important for determining the flow profiles near the beam window.

The curvature of the fillet at the base of the coolant-bounding wall was increased to $10 \mathrm{~cm}$ to streamline the flow in this area. Without the fillet, flow unsteadiness occurs downstream, which could lead to undesirable temperature and pressure fluctuations. The unsteadiness also leads to poor convergence of the steady-state solver, for which transient calculations must then be performed. The new fillet dimension greatly reduces this unsteadiness, and so it is used in the calculations.

The problem domain of the CFD analysis is shown in Figure 14. It is a 3-D, which provides more accurate results than the axisymmetric approach used in prior work. The domain features two symmetry planes in the azimuthal direction which approximates the behavior as having $1 / 12$ symmetry, sweeping out a $30^{\circ}$ sector. This captures the flow physics details. The helium zone around the target insulates the target from the subcritical assembly. For this reason, the target model was simulated as a stand-alone case, and the computational domain was truncated at the outer coolant wall. A domain with $1 / 4$ symmetry $\left(90^{\circ}\right.$ sector) was also tested for comparison. The obtained results are very similar.

Due to a small bulk temperature change, constant coolant thermal properties were used. Constant inlet temperature and velocity conditions were specified. No-slip conditions were enforced at all walls. Conjugate heat transfer was modeled at all material interfaces. Adiabatic boundaries were used on the inner window wall and the outer coolant wall, which should provide conservative temperature estimates. The k- $\Omega$ SST turbulence model with an "All-y+" hybrid wall modeling approach was primarily used. This model has historically shown good predictions for obtaining mean values in flows with separation. This should be expected to provide better results for predicting flow separation than the Standard k- $\varepsilon$ model with "High-y+" wall modeling that was used in prior work. A turbulent Prandtl number $\left(\mathrm{Pr}_{\mathrm{t}}\right)$ approach was used to model the turbulent heat flux. A constant value of 0.9 was used for most simulations. 


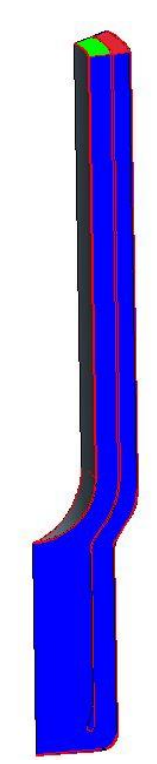

Figure 14 CFD domain with 1/12 symmetry.

Model meshing was performed with primarily polyhedral cells. A thickness of three cells was found to be sufficient resolution for the solids. The bottom portion of the target, near the beam window, was meshed with an unstructured approach. It was then extruded in the axial direction for efficient mesh generation, along the channel above the beam window. The mesh was refined in the separation region. A prismatic layer was generated on all fluid-facing walls such that $30<y+<300$.

A power mapping script was written to perform a nearest-neighbor interpolation from the MCNP mesh tally for the power distribution. This was written as FORTRAN user code, which was necessary since the native routines within STAR-CCM+ are currently unable to perform interpolation using cylindrical coordinates. The power was uniform azimuthally, with interpolation performed only in the R-Z plane. The power distribution is applied to all materials within the target. The profile is shown in Figure 15. The power peaks just below the beam window and extends further axially than radially. It is seen that there is heat deposition in the separation wall.

It is important to note that using different meshes affects both the CFD calculation as well as the local power distribution. Small changes can affect the local temperature distribution given the strong gradient of the power distribution, particularly near the beam window. For this reason, a mesh that solves the fluid/energy equations to acceptable accuracy might still see temperature changes with mesh refinement.

Results for three different mesh refinements for a $30^{\circ}$ sector case with $2 \mathrm{~m} / \mathrm{s}$ inlet velocity are given in Table IV. These consist of a coarse mesh (460K cells), base mesh ( $863 \mathrm{~K}$ cells), and fine mesh ( $3 \mathrm{M}$ cells). For all cases, the peak coolant outer wall temperature was $664 \mathrm{~K}$, while the outlet temperature was $711 \mathrm{~K}$ (bulk $\Delta \mathrm{T}$ of $\sim 60 \mathrm{~K}$ from inlet to outlet). It is demonstrated that mesh refinement has little impact on predicting the peak surface temperature of the beam window, and more impact on predicting the separation wall temperature. Accurate prediction of the flow separation also leads to a 
lower pressure drop result. The inner window temperature is important for thermal stress purposes, but the $873 \mathrm{~K}$ temperature limit does not apply given that it is not in contact with molten lead.

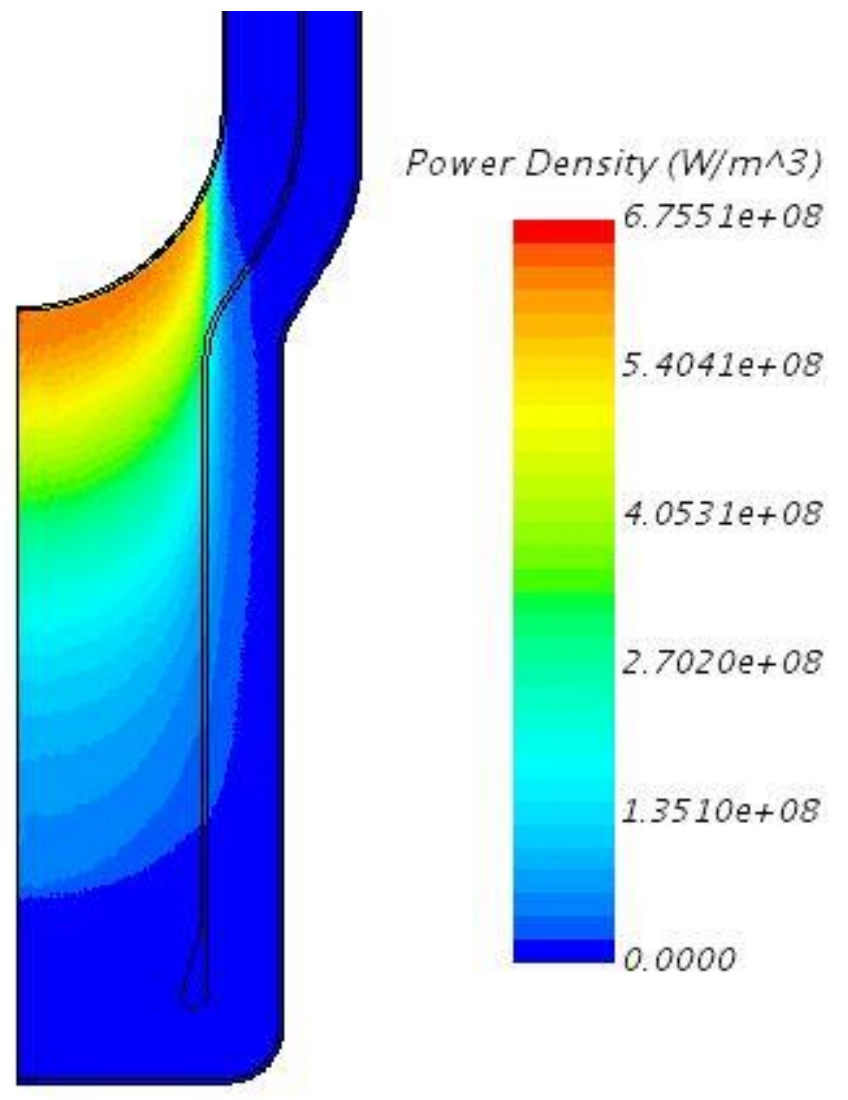

Figure 15 Power density $\left(\mathrm{W} / \mathrm{m}^{3}\right)$ for all target materials.

Table IV Comparison of key thermal hydraulic values with mesh refinement, Temperatures in $\mathrm{K}$, pressures in $\mathrm{KPa}$.

\begin{tabular}{|c|c|c|c|c|}
\hline $\begin{array}{c}\text { Mesh } \\
\text { Refinement }\end{array}$ & $\begin{array}{c}\text { Peak Inner } \\
\text { Window Surface } \\
\text { Temperature }\end{array}$ & $\begin{array}{c}\text { Peak Outer } \\
\text { Window Surface } \\
\text { Temperature }\end{array}$ & $\begin{array}{c}\text { Peak Separation } \\
\text { Wall } \\
\text { Temperature }\end{array}$ & Pressure Drop \\
\hline Coarse & 832.4 & 743.9 & 780.3 & 75.6 \\
\hline Base & 837.6 & 742.4 & 775.9 & 74.0 \\
\hline Fine & 835.2 & 743.1 & 771.0 & 71.6 \\
\hline
\end{tabular}

Results from the base case are shown in Figures 16 to 18 . In general, the temperatures are highest near the target region, as expected. The recirculation zone next the separation wall has relatively high temperature. Reattachment occurs near the curved portion of the wall. This is much further downstream compared to the results seen in previous work. There is a roughly $100 \mathrm{~K}$ margin to the $873 \mathrm{~K}$ temperature limit for the 
wetted window wall and the separation wall. Thus, the basic design of the target is confirmed to be acceptable.
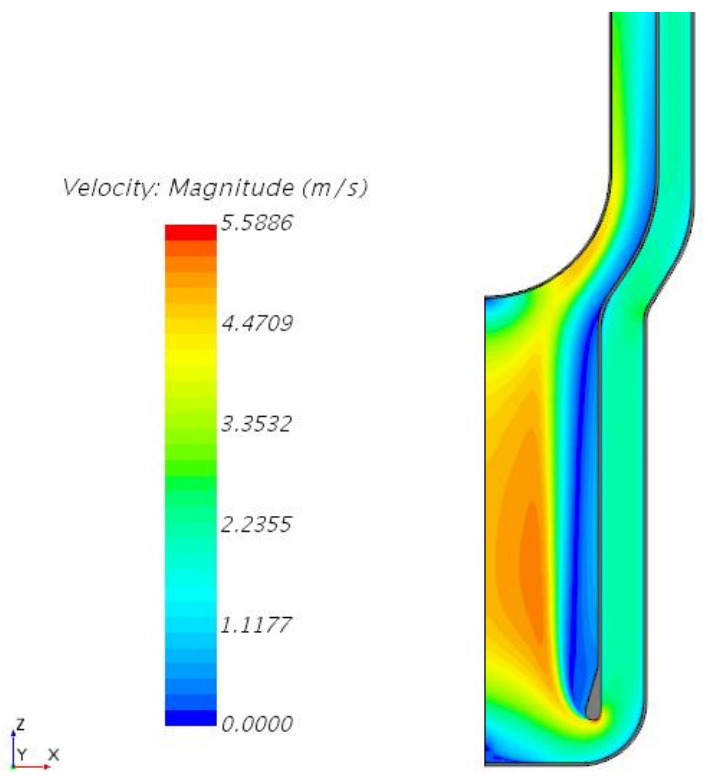

Figure 16 Velocity magnitude distribution at the symmetry plane for the base case.
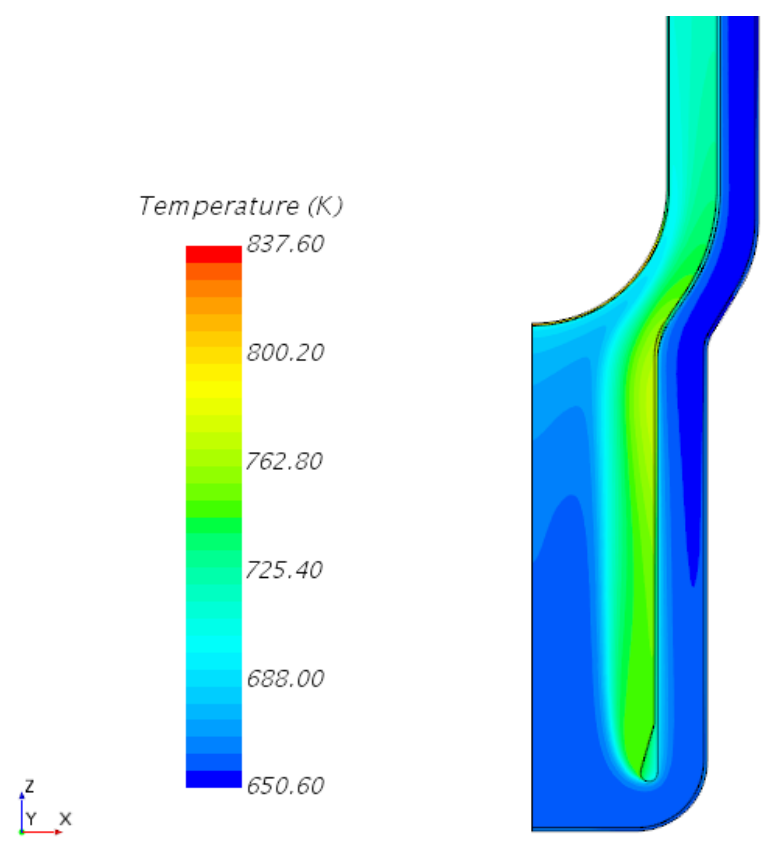

Figure 17 Temperature distribution at the symmetry plane for the base case. 

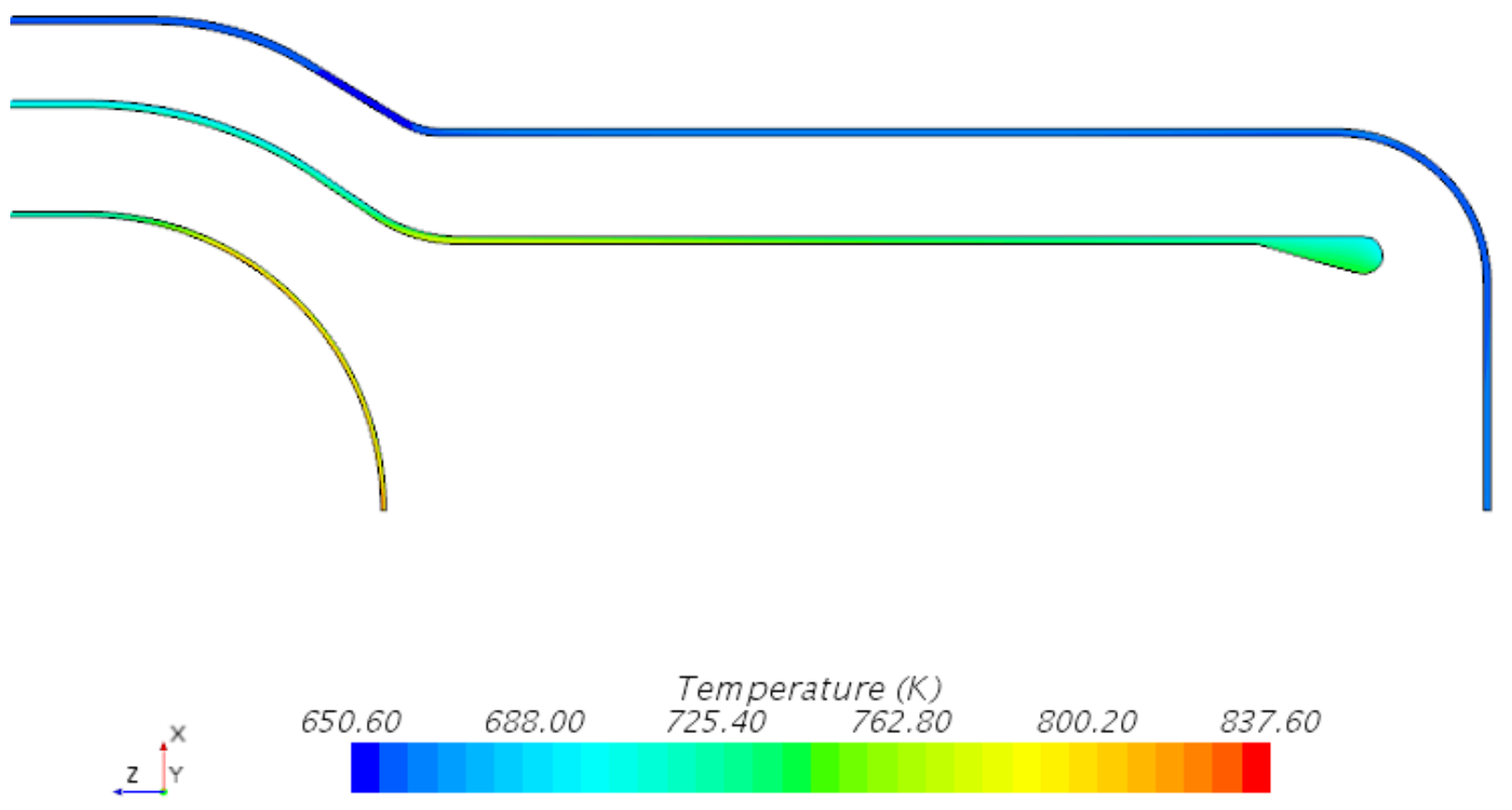

Figure 18 Structural temperatures at the symmetry plane for the base case.

A number of other cases were examined to assess the impact of uncertainties due to the model assumptions and selections. A summary of the results from the different cases is provided in Table V. The flow patterns from these cases are similar to the base case, and are not shown. The use of a $90^{\circ}$ sector vs. a $30^{\circ}$ sector had little impact on the calculation accuracy, but the $90^{\circ}$ sector allows for the creation of higher-quality cells near the radial center of the model. For this reason, the temperature predictions are improved while the flow predictions are similar. The "very fine" mesh case with this $90^{\circ}$ sector is used as the reference calculation, and it is highlighted in the table. This calculation shows a further reduction of pressure drop.

Another case was run using the standard $k-\varepsilon$ model as it was done in previous work. The velocity distribution from this case is provided in Figure 19. The recirculation zone is noticeably smaller than that of the SST model. This suggests that the flow separation predictions are inaccurate. In addition, it leads to lower temperature predictions. This implies that the peak temperatures in the previous work could have been underestimated. Another test case used the v2f turbulence model, which is known for giving improved predictions compared to other eddy-viscosity models, especially concerning near-wall behavior. These results corroborated the SST results, suggesting that the SST approach is reasonable. 
Table $\mathrm{V}$ Comparison of key thermal hydraulic values for various test cases, temperatures in $\mathrm{K}$, pressures in $\mathrm{kPa}$.

\begin{tabular}{|c|c|c|c|c|}
\hline Case & $\begin{array}{c}\text { Peak Inner } \\
\text { Beam Window } \\
\text { Surface } \\
\text { Temperature }\end{array}$ & $\begin{array}{c}\text { Peak Outer } \\
\text { Beam Window } \\
\text { Surface } \\
\text { Temperature }\end{array}$ & $\begin{array}{c}\text { Peak } \\
\text { Separation } \\
\text { Wall } \\
\text { Temperature }\end{array}$ & Pressure Drop \\
\hline "Very fine" reference & 832.5 & 750.7 & 771.1 & 66.6 \\
\hline $\begin{array}{c}\text { Standard k- } \varepsilon \text { model } \\
\text { "Very fine" V2F } \\
\text { model }\end{array}$ & 818.2 & 728.6 & 736.6 & 75.6 \\
\hline $\begin{array}{c}\text { Wider fairing } \\
\text { Pr }\end{array}$ & 829.0 & 751.8 & 791.5 & 67.8 \\
\hline $\begin{array}{c}\mathrm{Pr}_{\mathrm{t}}=2.0 \\
\text { fluid properties }\end{array}$ & 849.0 & 745.3 & 767.8 & 69.5 \\
\hline $3 \mathrm{~m} / \mathrm{s}$ inlet velocity & 813.3 & 716.4 & 738.0 & 74.0 \\
\hline
\end{tabular}

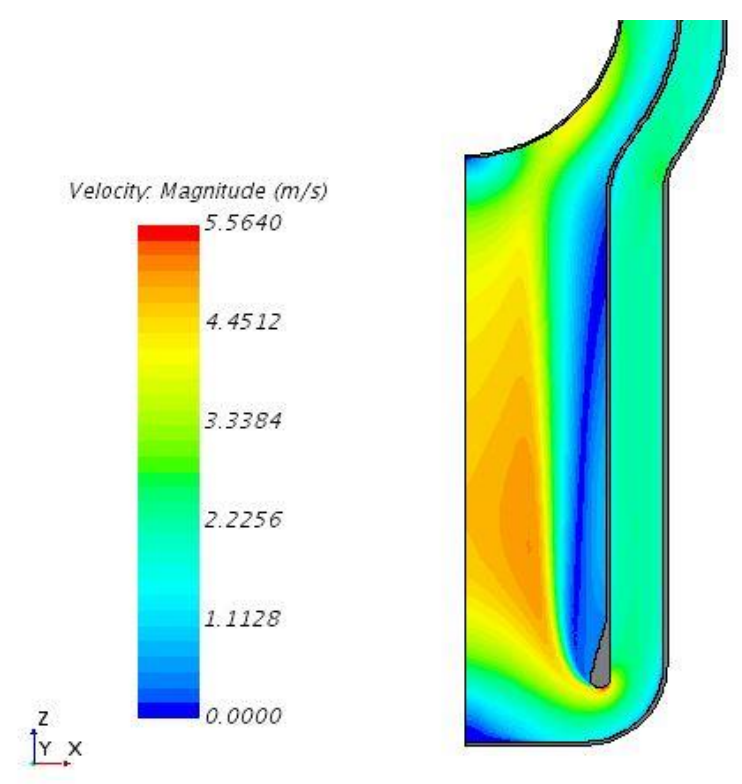

Figure 19 Velocity magnitude distribution at the symmetry plane for the Standard k- $\varepsilon$ case.

A number of cases were tested using different designs for the rounded fairing at the bottom of the separation wall. One case featured $\sim 2 x$ wider fairing than the base case. This yielded marginally higher window temperatures, but reduced separation wall temperatures. It reduced also the pressure drop by $\sim 6 \%$. This shows that the separation 
is more significant than that seen in the previous target design, and that the fairing design has a relatively small impact. Nevertheless, a wider fairing than in the base case should be utilized. Some investigations into uncertainties in the thermal properties were also undertaken. The effects of different $\mathrm{Pr}_{t}$ and variable properties were found to have only a small impact on the beam window temperatures.

Another simulation was performed with $3 \mathrm{~m} / \mathrm{s}$ inlet velocity since the liquid lead is less corrosive than LBE. The inlet velocity increase reduces the peak temperatures in the system. The separation wall temperature is reduced by $\sim 40 \mathrm{~K}$. However, there are some drawbacks to this increase. The higher mass flow rate reduces the outlet temperature to $691 \mathrm{~K}$, and subsequently the thermal efficiency. The more important issue is the pressure drop increase, which is doubled relative to the $2 \mathrm{~m} / \mathrm{s}$ case. This is a significant increase, but it demonstrates the possibility of lowering peak system temperatures. Given the large temperature margins calculated here with $2 \mathrm{~m} / \mathrm{s}$ inlet velocity, this value is considered acceptable.

In conclusion, steady-state thermal-hydraulic analyses of the target have been performed. Improved computational models were developed for studying the separation behavior, and a number of simulations were performed to study the modeling assumptions. The results show that the design geometry is appropriate, and the system temperatures are within the design limits.

\subsection{Homogeneous Configuration}

The first examined configuration is the homogenous concept. In this concept, the subcritical blanket has a cylindrical tank filled with the liquid metal slurry fuel and it is segmented radially to eight sectors. The target assembly is located at the tank center connected to a separate coolant loop. Eight heat exchangers are used for the eight subcritical blanket sectors with manifolds located at the top and the bottom of each sector. The slurry fuels are pumped by the electromagnetic pumps and circulated between the subcritical assembly and the outside heat exchangers to remove the fission power. The subcritical fission blanket uses the liquid lead as the fuel carrier. To eliminate the thermal power peak caused by the graphite reflector, a steel material is used for the reflector zone. Figure 20 plots one eighth of the subcritical blanket utilized in the MCNPX simulations. The subcritical assembly tank has no extra internal structure materials, except the structure walls separating the eight sectors of the tank. Therefore, the source neutrons generated from the spallation reactions are utilized effectively.

With simple heat transfer balance sheet, the required flow rate can be calculated to be $\sim 14.2 \mathrm{~m}^{3} / \mathrm{s}$ for removing the $3 \mathrm{GW}$ fission power for $140 \mathrm{~K}$ coolant temperature change. The average flow velocity is limited to $3 \mathrm{~m} / \mathrm{s}$. Thus, the total flow area is $\sim 4.7 \mathrm{~m}^{2}$. The heat transfer analysis shows that the necessary heat exchanger height is $\sim 7.3 \mathrm{~m}$. The total volume of the liquid metal mobile fuel outside the subcritical assembly tank is $\sim 70 \mathrm{~m}^{3}$, which is almost three times of the fuel volume inside the subcritical assembly tank. 


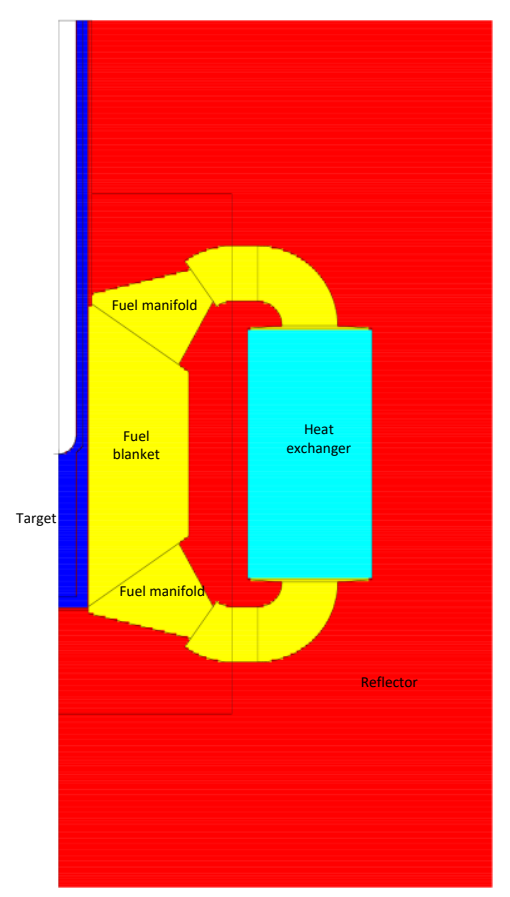

Figure 20 Monte Carlo Model of the Homogeneous Self-Cooled Blanket Configuration.

The slurry fuel compositions and the physical parameters of the homogeneous configuration are listed in Table VI. The plutonium concentration is increased to $45 \%$ to reduce the required MAs inventory. In this selected configuration, the required initial MAs inventory for starting this ADS system is $\sim 23$ ton. This MAs inventory is too large and it is not practical to use for several reasons. First, it requires processing 14,000 ton of the US spent nuclear inventory before starting the first ADS system. Such large processing inventory prolongs the required time for disposing of the spent nuclear fuel and does not permit the startup of other ADS burners. Second, in some accident scenarios, it is not desirable to have a large amount of MAs inventory in one system for safety considerations. Third, circulating the liquid metal mobile fuel outside the subcritical assembly tank provides the possibility for the fuel particles to precipitate on the cold surfaces of the cooling loop. Therefore, other configurations were examined to eliminate these concerns.

\subsection{Inverted Bundle Configuration}

To reduce the amount of the liquid mobile fuel volume in the ADS system and to eliminate the disadvantages of the homogenous configuration, ADS systems with separate primary coolant loops were investigated. Bundle tubes are inserted vertically in the subcritical assembly tank to simplify the structural design. The slurry fuel fills the subcritical assembly tank, and a small stream of the mobile fuel is slowly circulated outside the tank to control its chemical composition, remove the short-term fission products, and add MAs materials. The primary cooling loops use the liquid lead coolant inside bundle tubes and are connected to the heat exchanger outside the subcritical assembly vessel. It is called the inverted bundle model because in the heat transfer calculations, the coolant 
now is inside the tube and the fuel is outside the coolant tube. Such configuration represents the inversion of the conventional fission reactors.

Table VI Physical parameters of the homogenous configuration

\begin{tabular}{|l|c|}
\hline Total actinide oxide volumetric concentration in the slurry: & $4.46 \%$ \\
\hline Total uranium and plutonium atomic fraction in the actinide fuel: & $45.0 \%$ \\
\hline Target outer radius, cm & 34.27 \\
\hline Target proton beam energy, GeV & 1.0 \\
\hline Target proton beam current, mA & 25.0 \\
\hline Fission blanket outer radius, cm & 150.0 \\
\hline Fission blanket height, cm & $\sim 150.0$ \\
\hline Fission blanket volume (with manifolds), m3 & 25.8 \\
\hline Fission blanket neutron multiplication factor & 0.980 \\
\hline Fission power in the blanket, GW & 3.23 \\
\hline Estimated fuel slurry volume outside the blanket, m3 & $>70.0$ \\
\hline Total actinide initial fuel inventory, ton & $>41.3$ \\
\hline Total MAs fuel initial inventory, ton & $>22.7$ \\
\hline
\end{tabular}

The Monte Carlo model of the geometry where upper and lower manifolds are used for the coolant connections is shown in Figure 21. The target has a separate coolant loop to keep the subcritical assembly free from the spallation products. HT-9 steel is the structural material for the tank and the coolant tubes. Eight heat exchangers connected with the coolant tubes are used for removing the generated fission power. The coolant velocity at the steel surfaces is limited to $3 \mathrm{~m} / \mathrm{s}$.

In the subcritical assembly, the fission power density of the region close to the neutron source is high relative to the conventional power reactors. The power density distributions calculated with the Monte Carlo model at three different planes, at the midplane $z=0$, and $z= \pm 10 \mathrm{~cm}$ below and above the mid-plane, are shown in Figure 22-a. The power density distributions are also plotted along the axial direction at several radial positions $r=35.0,45.5,75.5,155.0$, and $185.0 \mathrm{~cm}$ in Figure 22-b.

The region close to the target with $\sim 35 \mathrm{~cm}$ radius has the highest power density. It is about 4 times higher than the average value close to the outside fuel tank boundary. The axial power density distribution has also a much stronger peak close to the target zone. The hot channel factor is $\sim 4.15$, which is typically $\sim 2.6$ for pressurized power water reactors.

To take account of the non-uniformity of the power distribution, more coolant tubes are used close to the target zone as shown in Figure 21. The coolant tubes are arranged 
with higher density around the target zone. In regions close to the target, the tube lattice is tight and the lattice pitch is slowly increased in the radial direction away from the target. The distances between the two adjacent rings of coolant tubes are adjusted to satisfy the maximum temperature requirements based on the heat transfer analyses. With the coolant tubes integrated into the subcritical assembly tank, the tank radius is $2.0 \mathrm{~m}$ and its height is $4 \mathrm{~m}$.

The physical parameters of two inverted bundle configurations with two different tube diameters are listed in Table VII. The tube wall thickness is $2 \mathrm{~mm}$ and the coolant tube positions are fine-tuned. The physics and the heat transfer analyses were iterated to achieve those conceptual configurations. To achieve 0.98 neutron multiplication factor, the system requires an increased amount of MAs loaded into the mobile fuel slurry to account for the neutron losses in the steel coolant tubes. In addition, these neutron losses reduce the total amount of fission power generated in the system compared with the total generated power in the previous homogenous configuration.

Table VII. Main parameters of the inverted bundle configuration

\begin{tabular}{|l|c|c|}
\hline Subcritical Assembly Configurations & I & II \\
\hline Actinide oxide volume fraction in slurry: & $8 \%$ & $8 \%$ \\
\hline U/Pu atomic fraction in the fuel: & $46.15 \%$ & $46.53 \%$ \\
\hline Target outer radius, cm & 34.27 & 34.27 \\
\hline Subcritical assembly tank outer radius, cm & 200 & 200 \\
\hline Subcritical assembly tank height, $\mathrm{cm}$ & 400 & 400 \\
\hline Subcritical assembly fuel volume, $\mathrm{m}^{3}$ & 29.63 & 30.13 \\
\hline Coolant tube inner radius, $\mathrm{cm}$ & 0.85 & 0.70 \\
\hline Number of coolant tubes & 13824 & 18336 \\
\hline Coolant and structure volume, $\mathrm{m}^{3}$ & 19.15 & 18.66 \\
\hline Effective neutron multiplication factor keff & 0.98 & 0.98 \\
\hline Generated fission power, GW & 2.55 & 2.50 \\
\hline Subcritical assembly fuel inventory, ton & 22.9 & 23.3 \\
\hline Subcritical assembly MAs inventory, ton & 12.4 & 12.5 \\
\hline
\end{tabular}

Figure 23 shows the calculated power density distributions for the first configuration. For the selected coolant tube pattern shown in Figure 21, the subcritical core geometry is azimuthally symmetric with respect to the first coolant tube next to the target zone. Therefore, the reactor physics and heat transfer analyses are performed for one wedge of the core with one coolant tube next to the target zone. As shown in Figure 23, the fission power is mainly deposited in the fuel zone with a very small fraction deposited directly in the coolant tubes. 


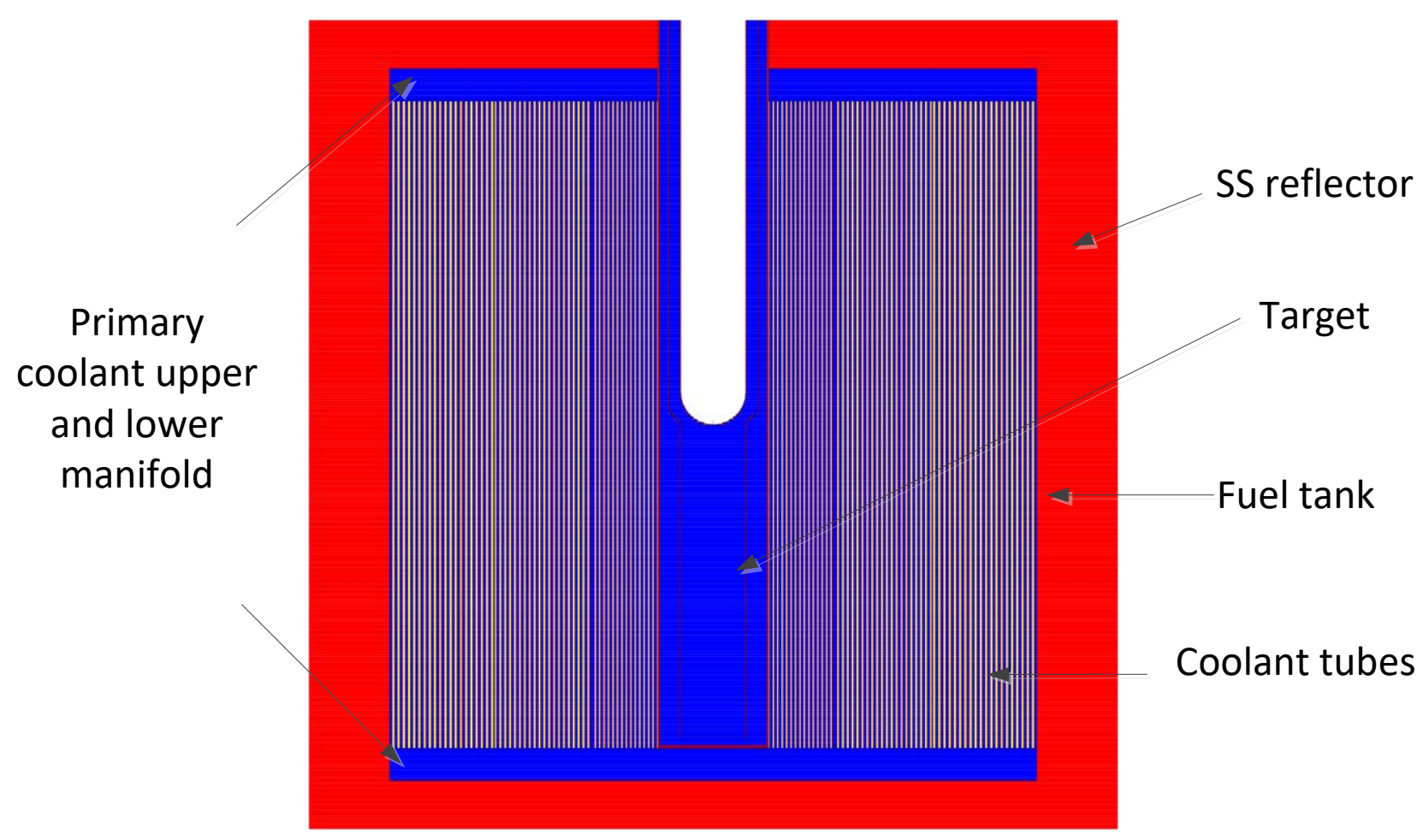

(a)

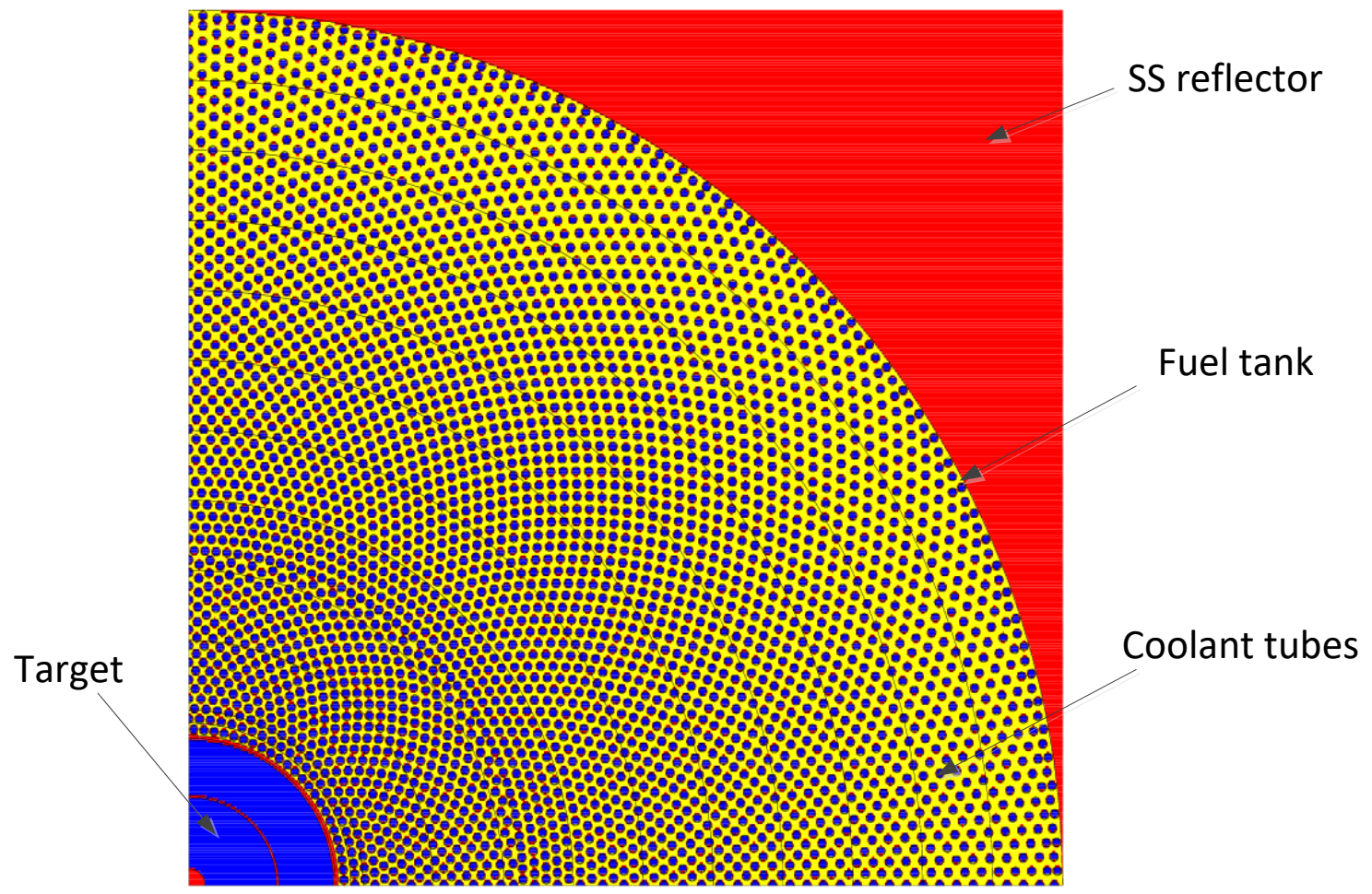

(b)

Figure 21 (a) X-Z view and (b) X-Y views of the Monte Carlo simulation model of the subcritical assembly of the inverted bundle design concept 


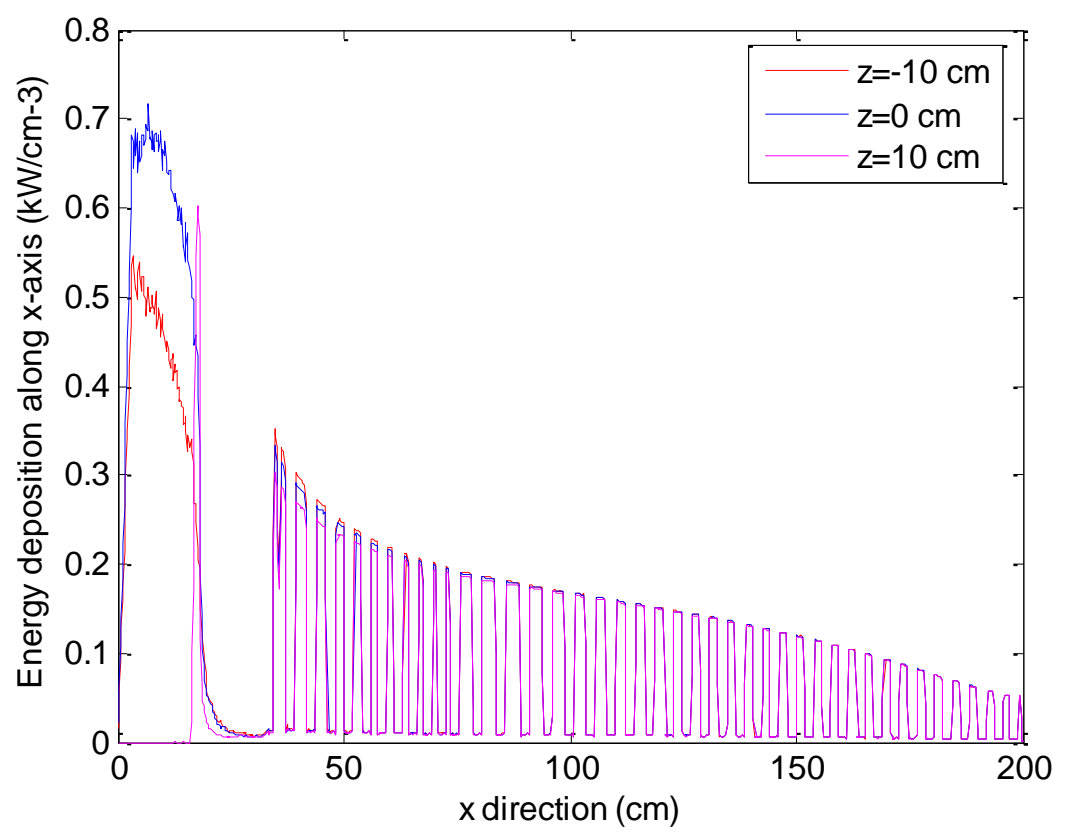

(a)

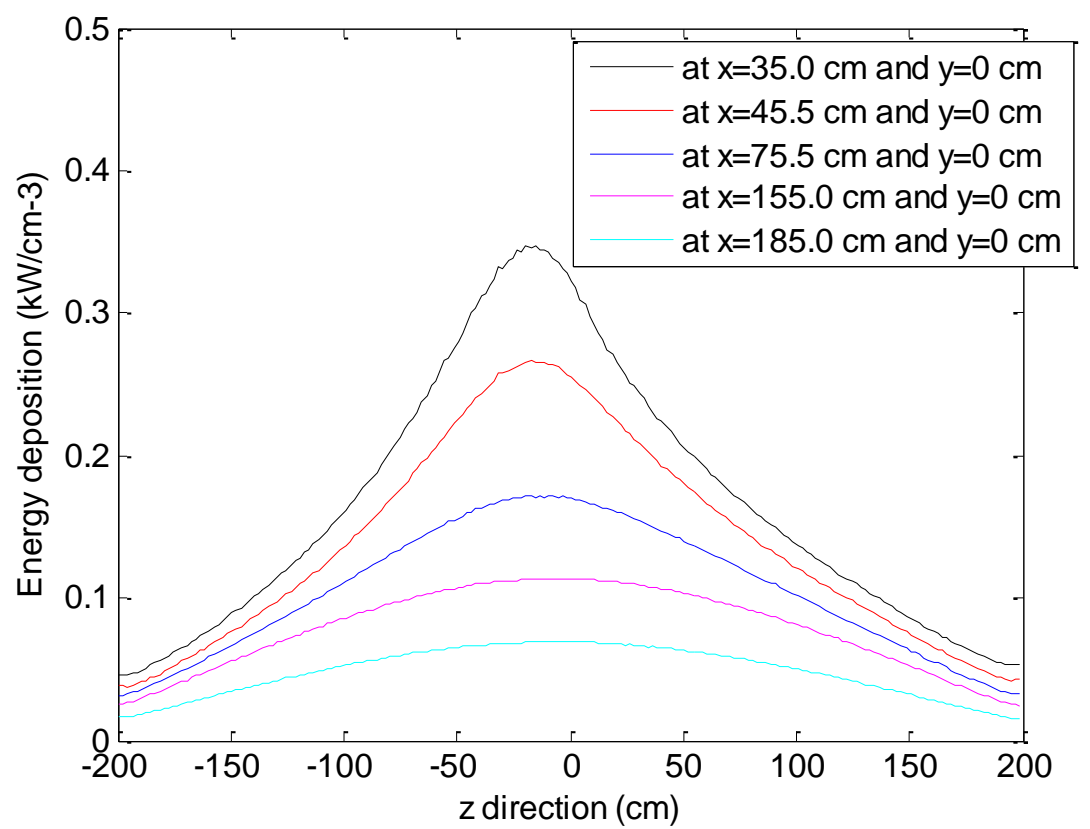

(b)

Figure 22 (a) The radial power density distribution at the middle plane and $\pm 10 \mathrm{~cm}$ below or above the middle plane (b) The axial power density distribution at the radial positions of $35.0,45.5,75.5,155.0$, and $185.0 \mathrm{~cm}$. 

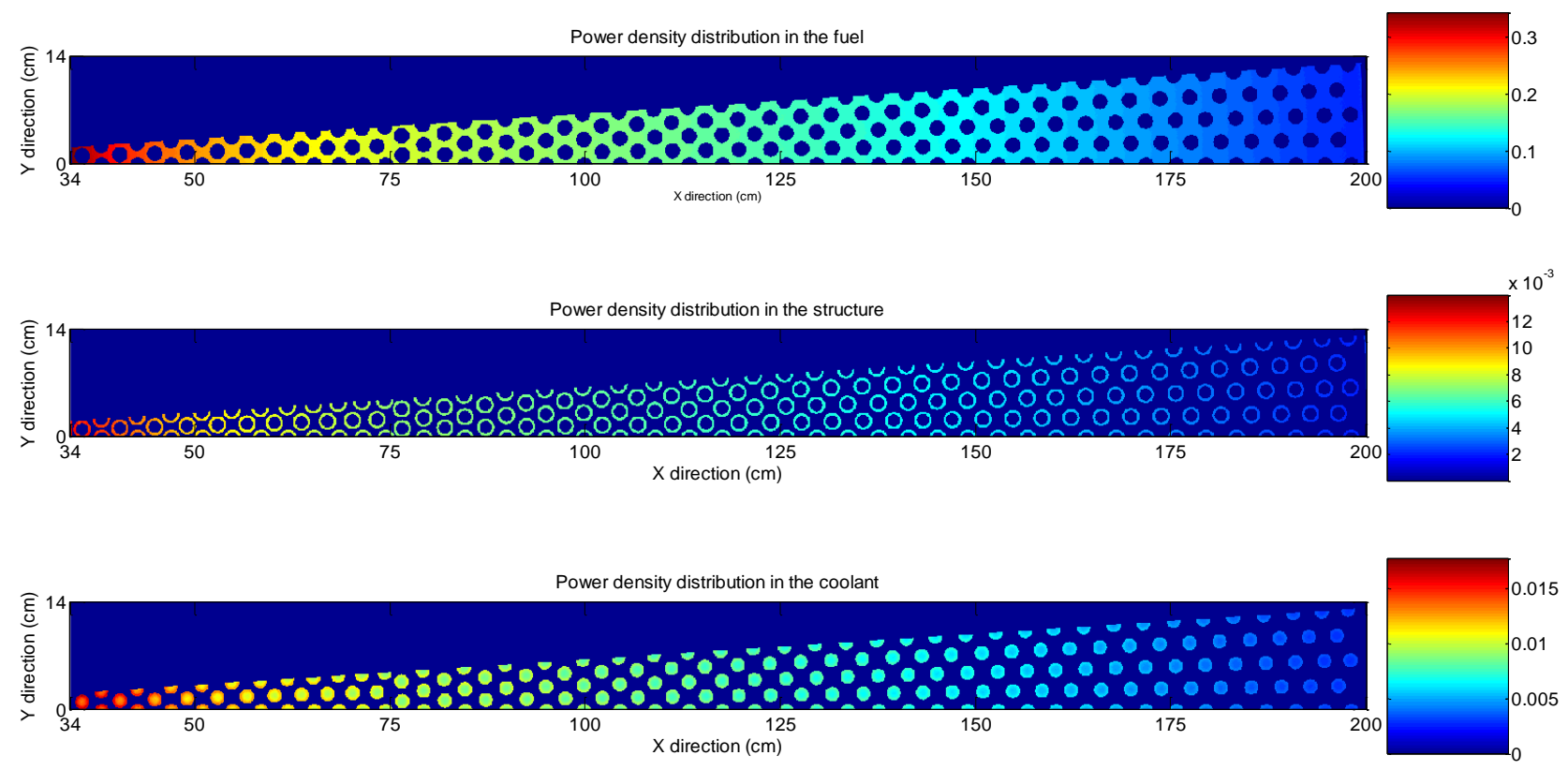

Figure 23 Energy deposition maps in the fuel, tube walls, and coolant for one wedge of the second subcritical assembly configuration in $\mathrm{kW} / \mathrm{cm}^{3}$.

Although using the wedge model has greatly reduced the geometrical model size, a large number of meshes is still required for the reactor physics calculations and the thermal hydraulics analyses due to the small size of each coolant tubes. In the MCNPX, about 200,000 meshes have been utilized to calculate the power distribution. The MCNPX reactor physics calculations and the STAR-CCM+ thermal-hydraulic analysis have been iterated many times in order to satisfy the design requirements for all the tubes.

In these iterations, some blanket parameters were fixed. The blanket outer diameter, the fuel tube diameter, and the coolant velocity were fixed. Typical limit for LBE coolant velocity is $\sim 2 \mathrm{~m} / \mathrm{s}$ to avoid corrosion/erosion of the steel structural material. However, the primary coolant is liquid lead, which is less corrosive than LBE. Therefore, the coolant average velocity for lead cases was fixed at $3 \mathrm{~m} / \mathrm{s}$. The other limiting design factor is the structure surface temperature of $\leq 600^{\circ} \mathrm{C}$, to limit the corrosion/erosion. Configurations with lead and LBE coolant materials were analyzed.

Initially the blanket was divided into three zones in order to establish different tube pitch based on the power density. Using a hexagonal lattice, 1/12 unit cell flow models with a highly-resolved structure region were constructed and simulated using the commercial Computational Fluid Dynamics (CFD) code STAR-CCM+ [14] to estimate appropriate tube spacing given the constraints listed above. The power distribution was based on results from MCNPX. Results from these CFD runs and corresponding MCNPX runs determined the general spacing and number of tubes. This step was only to provide a rough estimate of appropriate spacing before performing full optimization with a larger model. 
The spacing developed with the smaller CFD models was used for use in a better geometrical model and power profile representation. The smallest symmetry unit for the fission blanket was a "slice", shown in Figure 24. Since the flow is not homogeneous, full 3-D calculations are necessary. In addition, the cylindrical tank walls did not allow for a uniform P/D at the boundaries and a regular lattice pitch is not possible.

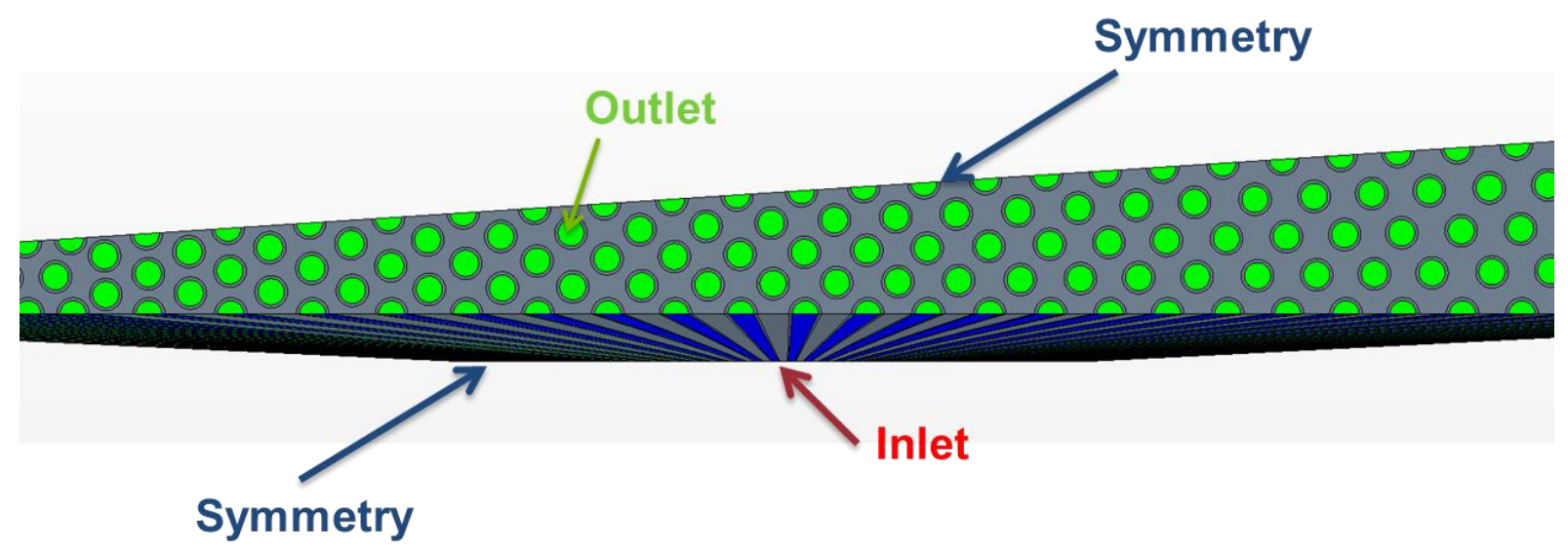

Figure 24 Minimal symmetry unit used in CFD simulations with some boundary conditions labeled.

It was advantageous to have a radially varying tube spacing reflecting the power density profile. The fission blanket optimization consisted of iterations between neutronics and thermal hydraulics. The power profile was provided from MCNPX and fed into STAR$\mathrm{CCM}+$, which in turn identified the maximum steel temperature for each coolant tube, highlighting areas with temperature above the design limit. The tubes were then rearranged based on these results and the process was repeated. Convergence to an optimal configuration, with uniform steel temperature distribution across the fission blanket was obtained within seven iterations.

The CFD calculations for this domain were very large, 40-60 million cells depending on the configuration. In order to expedite this process, a semi-automatic framework was developed to build, run, and post-process CFD simulations with minimal input data. A Java code was used to automatically build the geometry, set boundary conditions, mesh, and run the case based on the tube center coordinates. A FORTRAN code was employed to map the power from MCNPX tallies onto the STAR-CCM+ mesh. A final Java code was written to calculate thermal expansion, the maximum wall temperature, and outlet temperature for each tube. The results were used in the neutronics calculations and new tube positions were established. Despite this automation, the full iteration took about 10 calendar days on the average.

The inlet temperature for the LBE coolant was $590 \mathrm{~K}$. A realizable $\mathrm{k}-\varepsilon$ turbulence model was used. For the initial optimization iterations, simple boundary conditions were used. In Figure 24, the lateral boundaries had a symmetric boundary condition, inner and outer walls were adiabatic, and the bottom and top planes were inlet and outlet boundaries, respectively. An adiabatic boundary condition was used for the top and 
bottom surfaces of the fuel and the clad, which is conservative assumption. The reflector was treated as infinite. The fuel was also treated as solid due to its low velocity, which can be regarded as conservative assumption for cladding peak temperature. Given lower power densities in the clad and the coolant, power was initially only mapped to the fuel. A detailed computation with power mapped to all regions and realistic reflector size $(1 \mathrm{~m}$ in all directions) was performed for the optimal configuration.

A mesh was generated for the fuel, clad, and coolant regions, as shown in Figure 25, which was conformal at all material interfaces. At least three points were contained radially within the clad. A prismatic layer was included on walls touching the fluid surface, which ensured that the wall $y+$ was between 30 and 100 throughout the fluid domain.

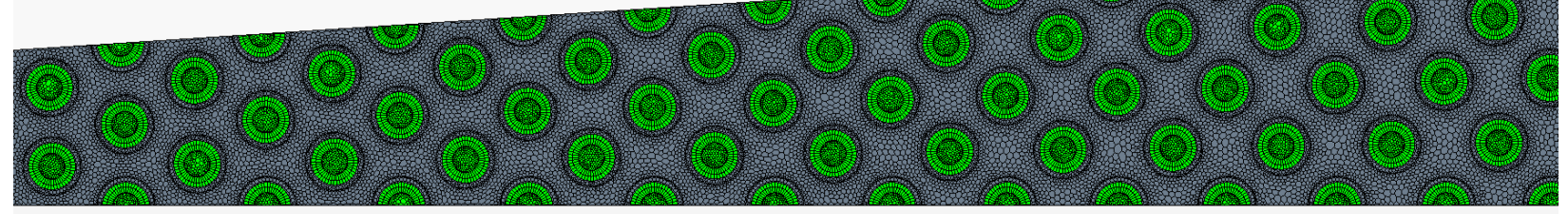

Figure 25 CFD mesh for the minimal symmetry unit.

A typical temperature distribution at a planar slice is provided in Figure 26. Some of the local jumps in temperature are due to tube pattern transitions. The peak temperature near the outer wall is due to the adiabatic boundary condition there, infinite reflector modeling, and the lack of space for more coolant tubes. Small dummy tubes could be placed there to reduce the fuel temperature without sacrificing much of the power. Although the temperature is high there, it is well within acceptable limits for the fuel.

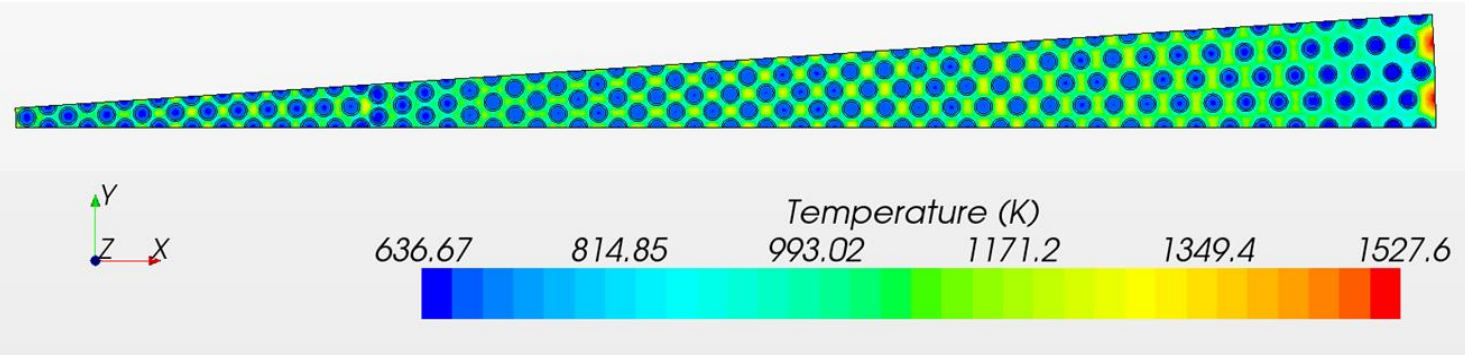

Figure 26 Sample temperature distribution for the minimal symmetry unit.

Different distributions of the same set of tubes can lead to different peak temperatures as shown in Figure 27, as well as the temperature profile of the optimal design. Figure 28 demonstrates the effect of using smaller tubes. More tubes must be used due to the constraint on coolant velocity, but a higher power distribution and lower temperature distribution can be obtained. 

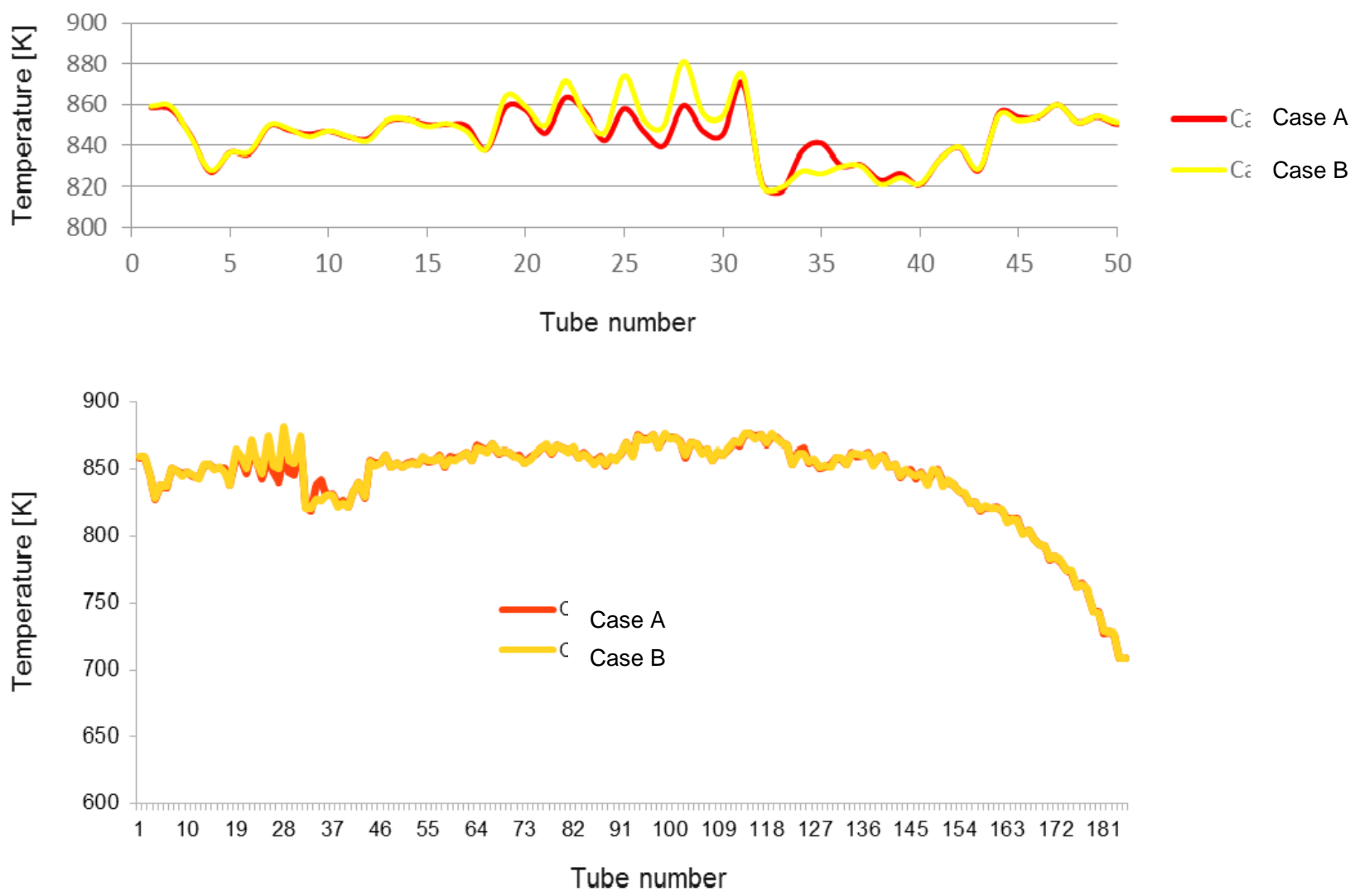

Figure 27 Peak cladding temperature distribution across the domain. Tube number increases with increasing radial coordinate. Case $A$ is the optimal configuration.

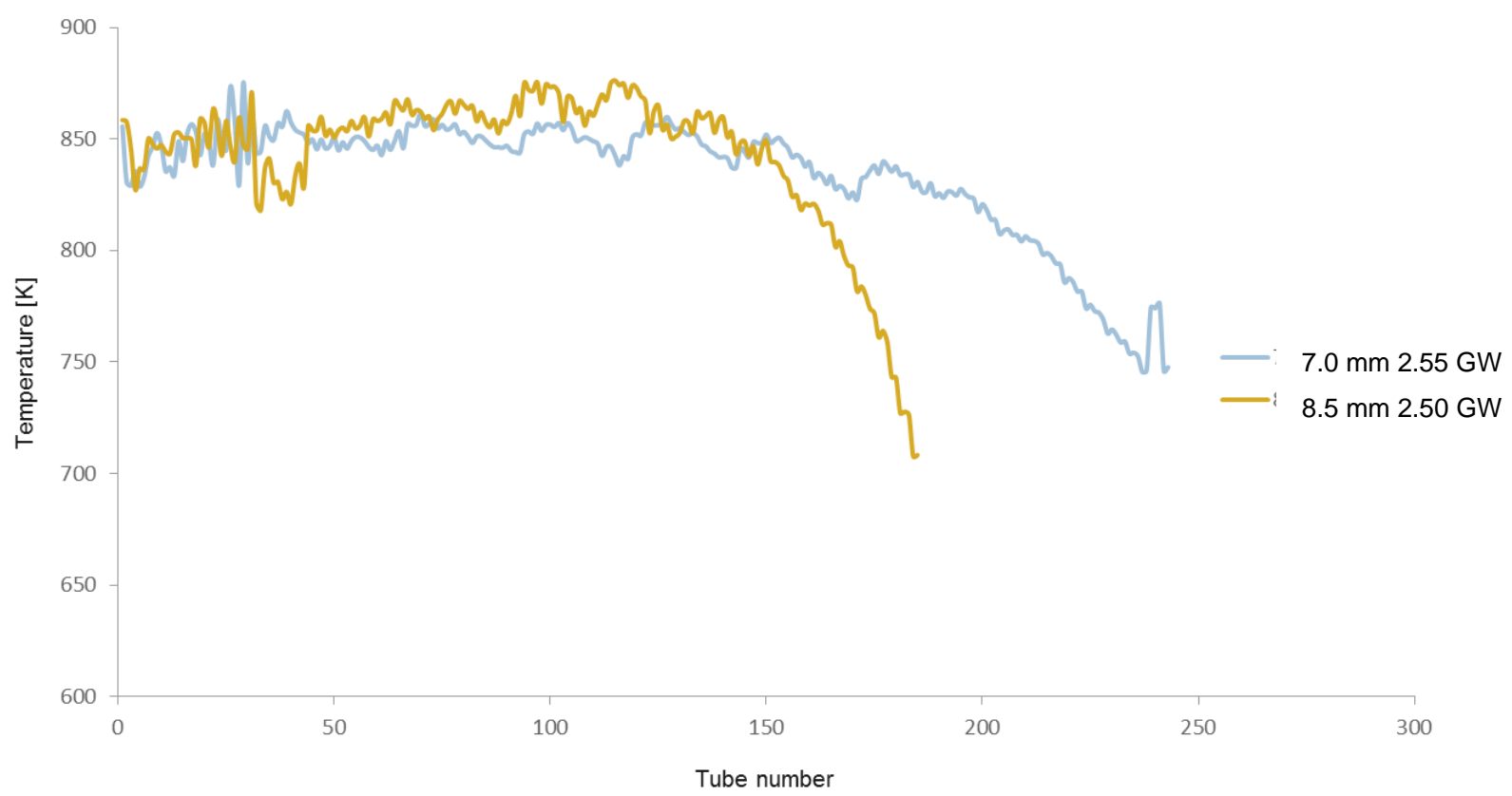

Figure 28 Radial temperature distribution for two cases with different tube radii. Note the higher power but comparable temperatures for the $7 \mathrm{~mm}$ case. 
After obtaining the optimal tube distribution, full mapping of the power from the MCNPX mesh to the STAR-CCM+ mesh was performed for accurate temperature distribution. However, the used meshes of the two codes are different, as shown in Figure 29. MCNPX has a uniform hexahedral mesh, which is imposed upon the geometry, while STAR-CCM+ has an unstructured and non-uniform mesh that follows the geometry. In order to avoid inaccuracies at the material boundaries, a separate mesh tally (using the same grid) was performed separately for the coolant, the clad, and the fuel. This yielded three separate power density distributions, which were mapped independently to each of the STAR-CCM+ regions. Integration of the three power density distributions on the CFD mesh results in the same MCNPX power density distribution within $\pm 0.3 \%$. The power profiles in the CFD regions are shown in Figure 30. The power density of the coolant and the steel clad is more than an order of magnitude less than the fuel power density.

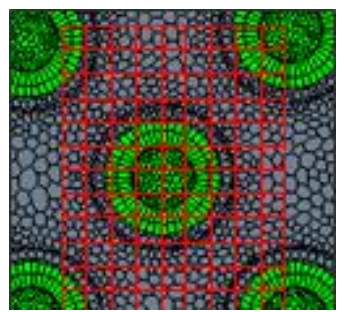

Figure 29 Differing meshes for power mapping. Red is the MCNPX uniform mesh (for illustration only, not to scale), gray and green are the unstructured CFD mesh.

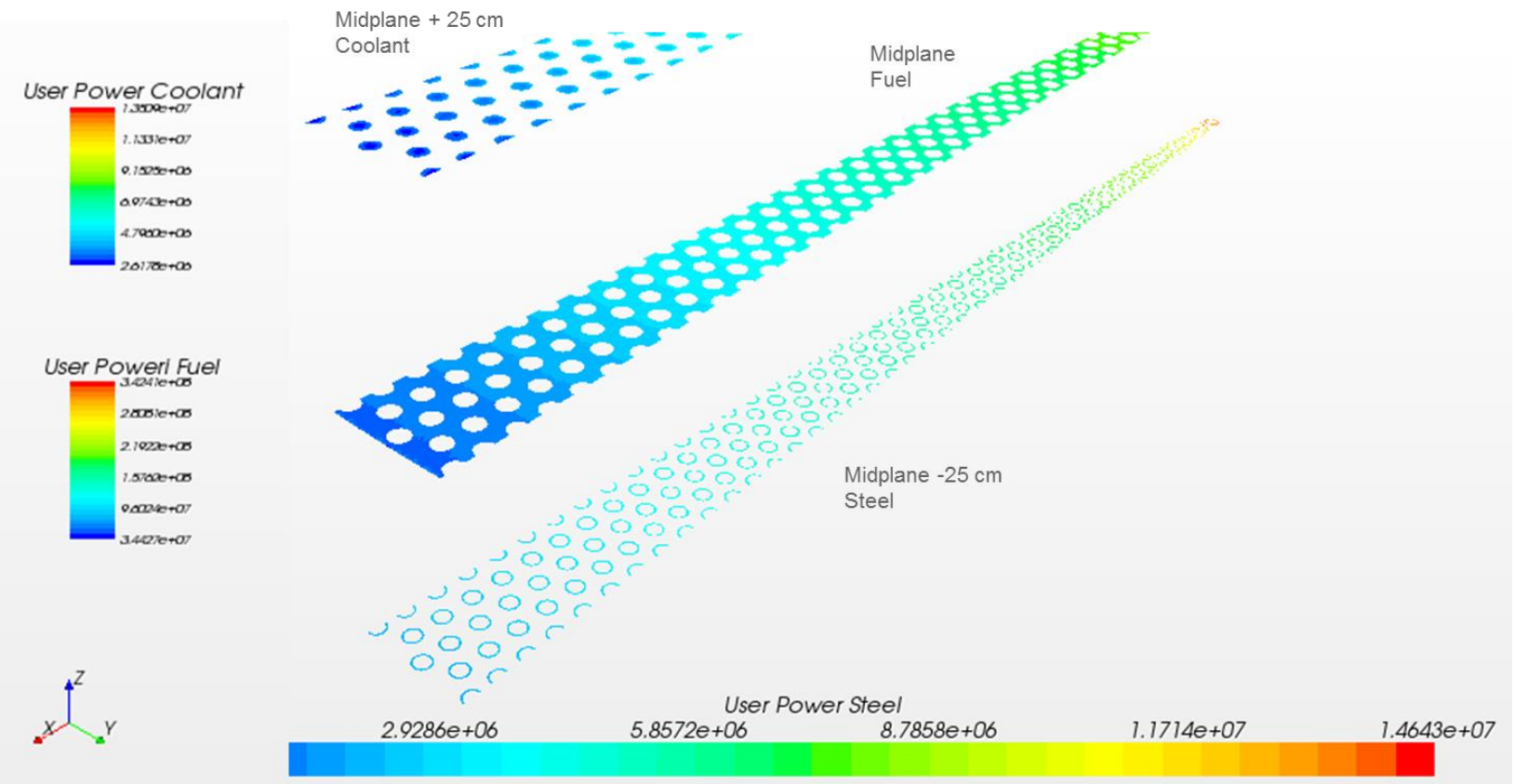

Figure 30 Power profiles mapped onto the CFD mesh for fuel, clad, and coolant regions.

Key parameters of the CFD model is listed in Table VIII. The increase in peak clad temperature due to the generated power in the clad and the coolant is shown in Figure 31. 
The calculated ISO temperature surfaces are shown in Figure 32. The temperature peaks near the axial center of the channels are due to the peaked axial power distribution.

Table VIII Geometry and flow parameters for the full power CFD simulation.

\begin{tabular}{|l|c|}
\hline \multicolumn{1}{|c|}{ Parameter } & Value \\
\hline Number of tubes & 183 \\
\hline Total fuel Power, MW & 26.045 \\
\hline Total Power, MW & 3 \\
\hline Coolant velocity, $\mathrm{m} / \mathrm{s}$ & 7.53 \\
\hline Mass flow rate per tube, $\mathrm{Kg} / \mathrm{s}$ & 21 \\
\hline Outer tube diameter, $\mathrm{mm}$ & 2 \\
\hline Tube wall thickness, $\mathrm{mm}$ & 1.6 \\
\hline Friction pressure drop, bar & 590 \\
\hline Inlet coolant temperature, $\mathrm{K}$ & \\
\hline
\end{tabular}

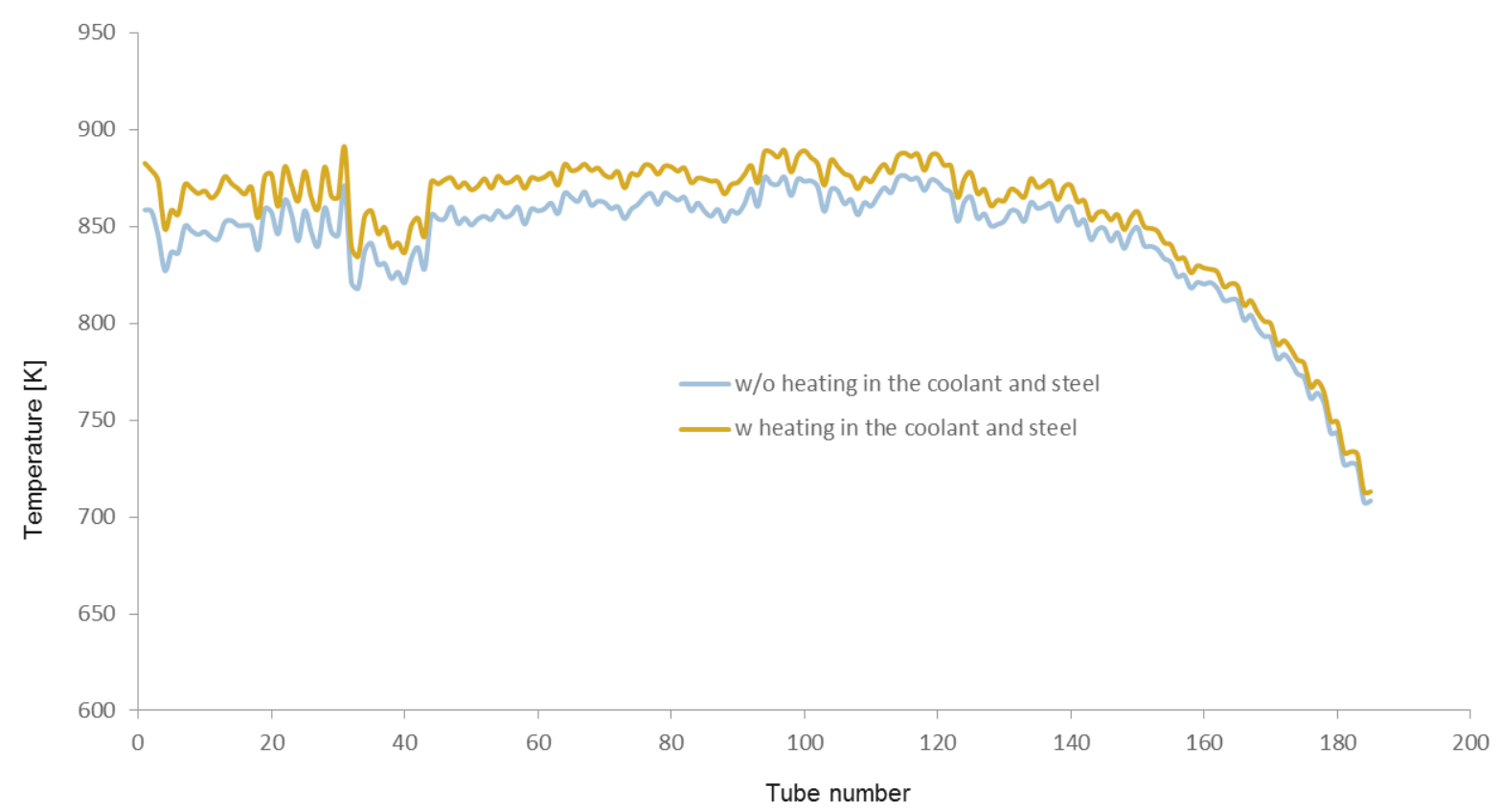

Figure 31 Peak cladding surface temperature comparison for cases with and without heating in the coolant and cladding included. 

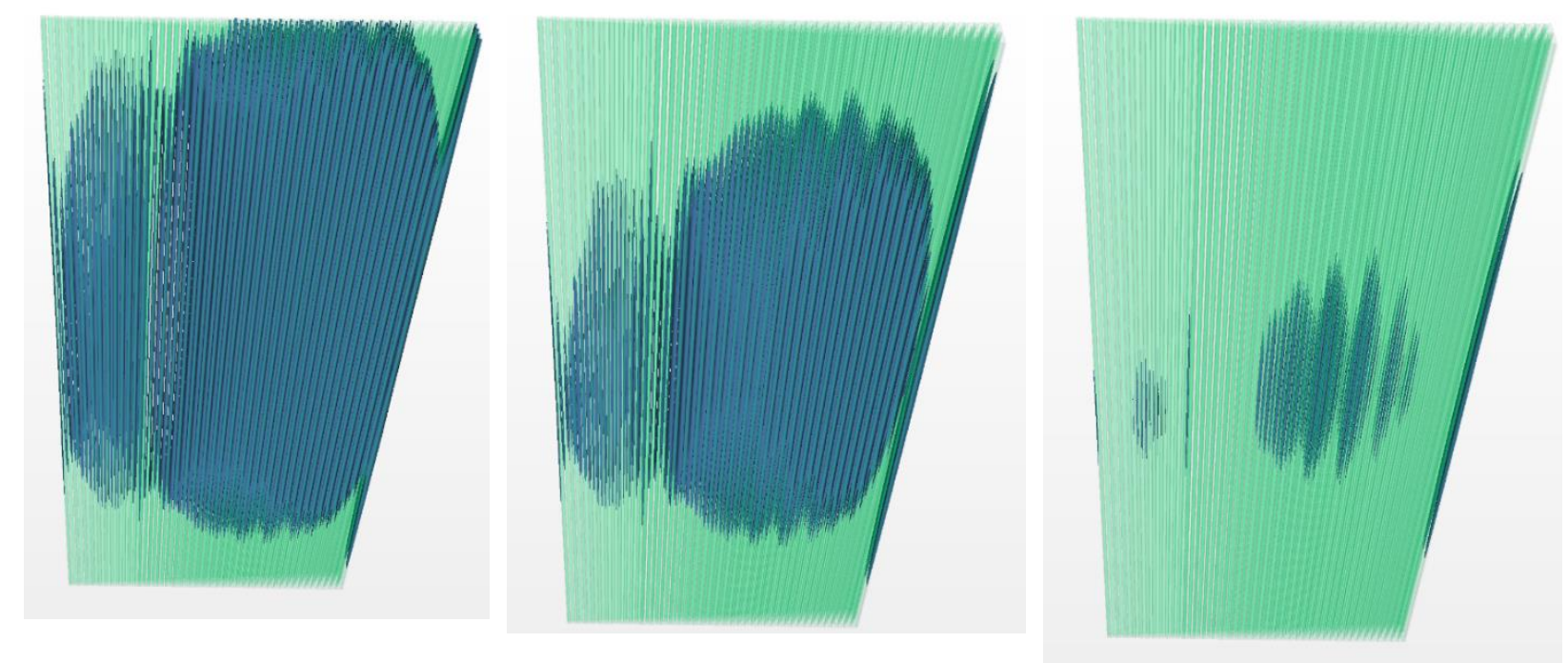

Figure 32 ISO Temperature surfaces for the full power, optimal case. Clockwise from left: $873 \mathrm{~K}, 973 \mathrm{~K}$, and $1173 \mathrm{~K}$.

The results from these simulations showed that the inverted bundle configuration could be designed to satisfy the design guidelines. Figure 33 shows the maximum surface temperatures of each coolant tubes in the two design concepts listed in Table VII and obtained from the coupled analyses. The second design concept satisfies the design criteria. The maximum surface temperatures of the coolant tubes are $\leq 600{ }^{\circ} \mathrm{C}$.

Compared with the previous homogeneous model, the inverted bundle model utilizes much less initial MAs materials. The fuel slurry volume is significantly reduced. If the 80,000 Metric Ton Heavy Metal (MTHM) in the SNF can be processed within 40 years, the first ADS system can start in four years if this configuration is used. However, as shown in Table VII, the inverted bundle model utilizes tens of thousands of coolant tubes, which are thin and each is $4 \mathrm{~m}$ long. This represents a difficult engineering challenge to keep the correct spacing between these tubes taken into consideration the different thermal expansion. In addition, the required number of spacers is excessive, which affects the performance of the subcritical assembly.

\subsection{Bundle Conceptual Configuration}

An improved bundle concept uses larger tube sizes and larger spacing is considered. In this concept, the slurry fuel is inside the tubes and the coolant is outside the tubes filling the subcritical assembly. Those bundle tubes are arranged in a triangular lattice and the lattice pitch is $4 \mathrm{~cm}$. To reduce the non-uniformity of the power distribution, the fuel tubes close to the target zone have a smaller diameter to increase the coolant/fuel volume ratio near the target. The fuel tube diameter gradually increases as the power density decreases away from the target zone. 


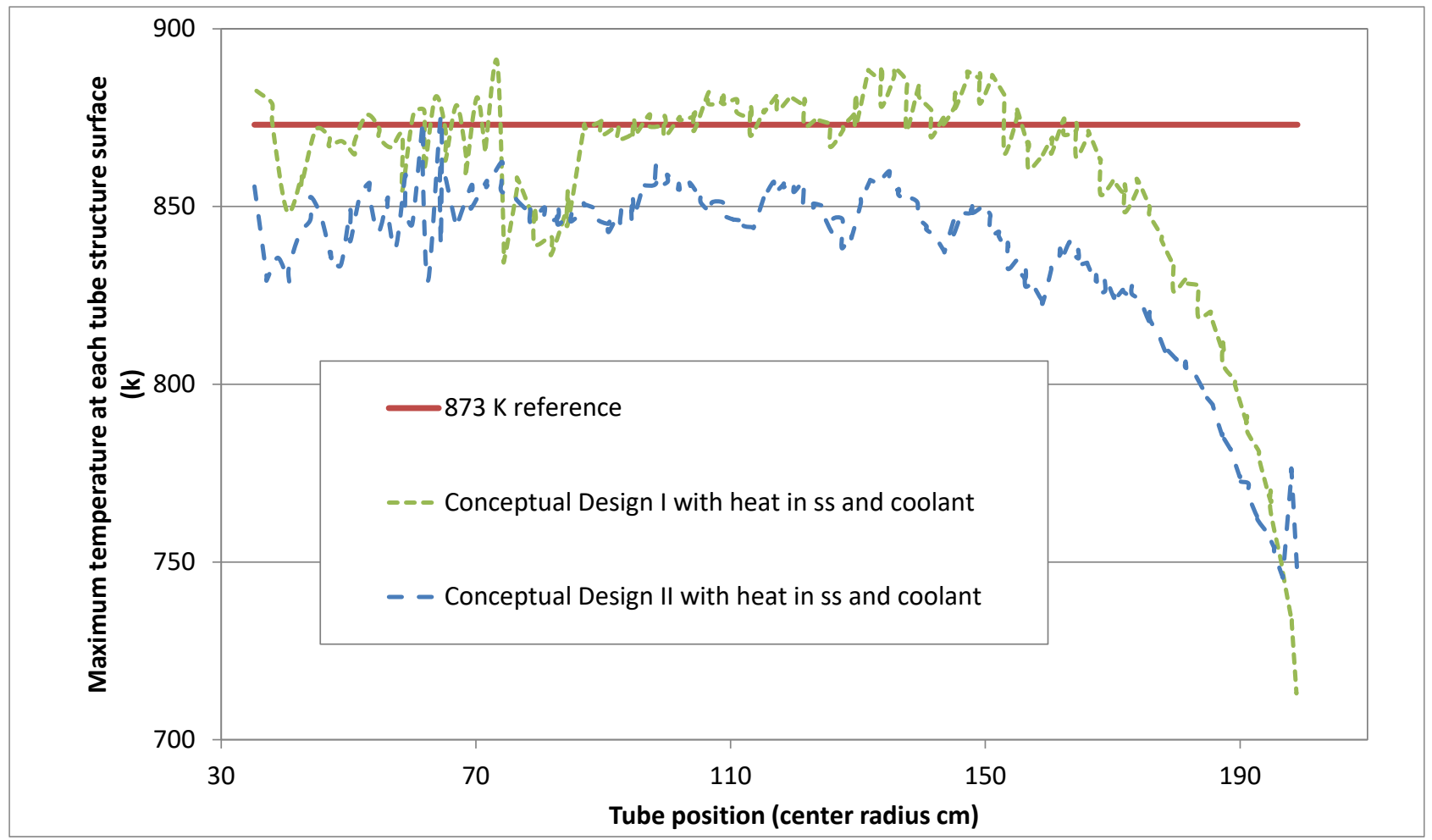

Figure 33 Calculated maximum surface temperature of each coolant tube for both design concepts.

The primary coolant is connected to the outside heat exchangers through the top and bottom manifolds. The bundle tubes are connected to each other at both ends with the slurry fuel slowly circulated outside the subcritical assembly for chemical control, removal of the short-lived fission products, and material feeding. The cylindrical tank is divided into eight or six sectors depending on the particular design and each sector is connected to a heat exchanger. The reactor physics analysis and the thermal hydraulic analysis were performed for half sector talking advantage of the symmetrical conditions. The first model has eight sectors as shown in Figure 34. The hexagon lattice pitch is 4.0 $\mathrm{cm}$, and a minimum lattice pitch to tube diameter (P/D) ratio is 1.25 as shown in Table IX. The tube thickness is $0.15 \mathrm{~mm}$.

The hot channel factor is about 3.9, which is very similar to the inverted bundle model. To accommodate this power density distribution, the fuel tube diameter is adjusted so the maximum tube surface temperature is less than $600^{\circ} \mathrm{C}$. The tube diameter is 2.8 $\mathrm{cm}$ when the tube is close to the target zone. The outer tube diameter increases to 3.2 $\mathrm{cm}$ when it is near the outer boundary of the fission blanket tank as shown in Table IX.

A single-channel heat-transfer analysis is performed for the hottest fuel tubes inside the subcritical fission blanket shown in Figure 34(b) with different diameters. The axial power density distributions of the four fuel tubes are shown in Figure 35. The calculated maximum surface temperatures of the four fuel tubes are listed in Table $\mathrm{X}$. The maximum surface temperature exceeds the designed limit of $600^{\circ} \mathrm{C}$ by $\leq 31^{\circ} \mathrm{C}$. 


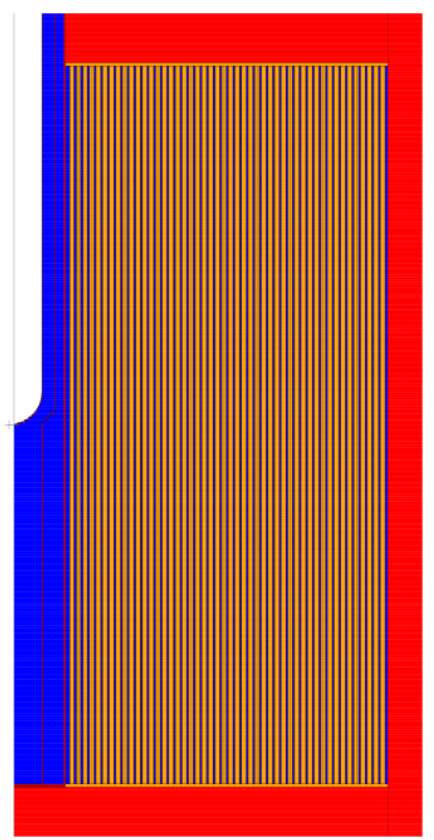

(a)

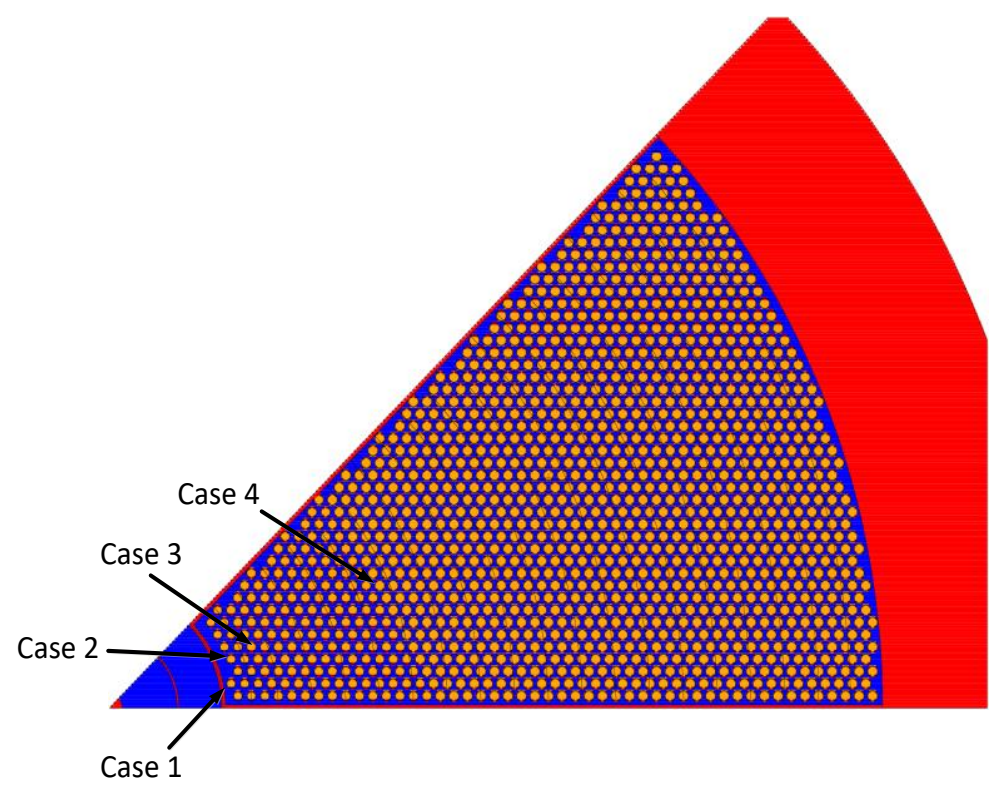

(b)

Figure 34 Vertical and horizontal cross section of the bundle conceptual configuration consists of one eighth of the subcritical fission blanket.

Table IX Main physical parameters of the bundle conceptual configuration.

\begin{tabular}{|l|c|}
\hline Total actinide oxide volumetric concentration in the slurry: & $9 \%$ \\
\hline Total uranium and plutonium atomic fraction in the actinide fuel: & $47.3 \%$ \\
\hline Target outer radius (cm) & 34.27 \\
\hline Target proton beam energy (GeV) & 1.0 \\
\hline Target proton beam current (mA) & 25.0 \\
\hline Fission blanket outer radius (cm) & 229.0 \\
\hline Fission blanket height (cm) & $\sim 440$ \\
\hline Fission blanket slurry fuel volume (m3) & 31.1 \\
\hline Tube inner radius (cm) & 1.25 to 1.45 \\
\hline Number of tubes & 10992 \\
\hline Liquid Pb coolant + HT-9 structure volume (m3) & 38.96 \\
\hline Fission blanket neutron multiplication factor & 0.980 \\
\hline Fission power in the blanket (GW) & 2.88 \\
\hline Total actinide initial fuel inventory (ton) & 27.6 \\
\hline Total MAs fuel initial inventory (ton) & 14.6 \\
\hline
\end{tabular}




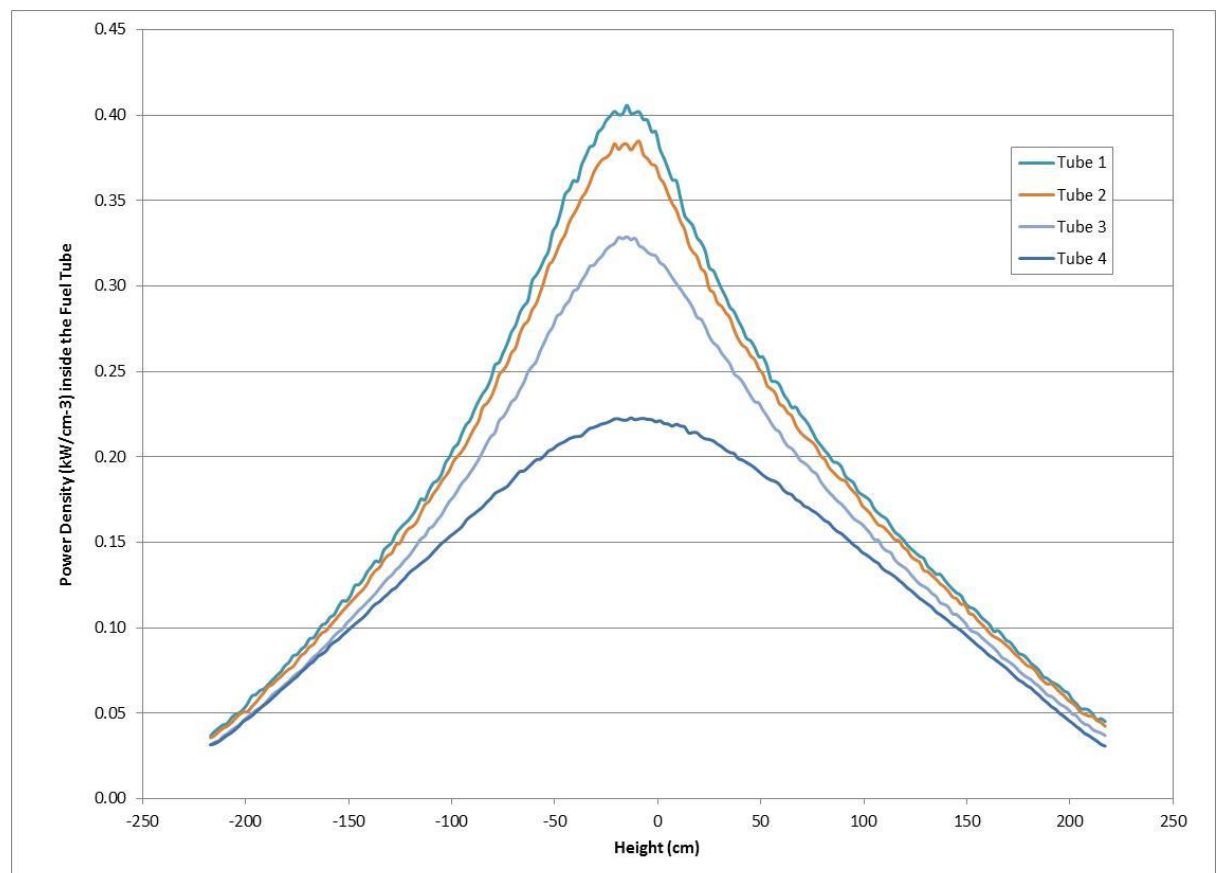

Figure 35 Axial power density distributions of the selected four fuel tubes shown in Figure 34(b) of the conceptual bundle configuration.

Table X Main parameters of MAs conceptual design using the bundle model.

\begin{tabular}{|c|c|c|c|c|c|c|c|}
\hline $\begin{array}{c}\text { Tube } \\
\text { Number }\end{array}$ & $\begin{array}{c}\text { Fuel } \\
\text { tube } \\
\text { power, } \\
\text { KW }\end{array}$ & $\begin{array}{c}\text { Structure } \\
\text { tube } \\
\text { power, } \\
\text { KW }\end{array}$ & P/D & $\begin{array}{c}\text { Tube } \\
\text { outer } \\
\text { diameter, } \\
\text { cm }\end{array}$ & $\begin{array}{c}\text { Tube } \\
\text { thick., } \\
\text { cm }\end{array}$ & $\begin{array}{c}\text { Tube } \\
\text { height, } \\
\text { cm }\end{array}$ & $\begin{array}{c}\text { Maximum } \\
\text { surface } \\
\text { Temp., K }\end{array}$ \\
\hline 1 & 414 & 2.70 & 1.429 & 2.8 & 0.15 & 436 & 904 \\
\hline 2 & 397 & 2.66 & 1.429 & 2.8 & 0.15 & 436 & 893 \\
\hline 3 & 414 & 2.86 & 1.333 & 3.0 & 0.15 & 436 & 901 \\
\hline 4 & 393 & 2.61 & 1.250 & 3.2 & 0.15 & 436 & 878 \\
\hline
\end{tabular}

The fission blanket is divided into radial sectors (six or eight sectors) by steel walls and each sector has its own coolant loop. This configuration does not allow for a small symmetrical unit less than half of a coolant sector, i.e. no less than $1 / 12$ or $1 / 16$ of the fission blanket. There are hundreds of fuel tubes in each sector and the CFD analysis of such half sector is very expensive during the early design iterations. Therefore, a simple approach was used during the early design iterations.

A Single-Channel Analysis (SCA) method was employed for estimating the temperatures for a given fuel tube. It assumes only radial conduction in the fuel and clad materials. In addition, the fuel is assumed solid, which is a conservative assumption. This assumption is appropriate since the fuel velocity is very low. The heat rates were derived from MCNPX energy deposition results for the fuel and the clad materials. Mixing between the channels was not included for providing conservative estimates. 
The main uncertainty is related to the selection of an appropriate Nusselt number correlation for coolant convection analysis. Many studies were performed for the heat transfer of low- Prandtl number fluids in rod-bundle geometries, but they must be applied with some caution to the current design. Most experiments were done with relatively fewpin arrangements of triangular pitch simulating fuel assemblies. Generally, the correlations are valid for Peclet number less than 5000, which is exceeded in the current design. For these reasons, CFD analyses are needed to validate the SCA results.

The Westinghouse correlation [22] was chosen the SCA analyses:

$$
N u=4.0+0.33\left(\frac{P}{D}\right)^{3.8}\left(\frac{P e}{100}\right)^{0.86}+0.16\left(\frac{P}{D}\right)^{5.0}
$$

$\mathrm{Nu}$ is the Nusselt number; $\mathrm{Pe}$ is the Peclet number; and $\mathrm{P}$ and $\mathrm{D}$ are the rod pitch and outer diameter; respectively. The correlation is valid for $1.1 \leq \mathrm{P} \leq 1.4$ and $10 \leq \mathrm{Pe} \leq 5000$. This correlation has the highest $\mathrm{Pe}$ limit that was found. In light of the above approximations, the primary purpose of the SCA is to obtain a rough estimate of temperatures so that a reasonable configuration can be defined before performing detailed CFD analyses.

The design constraints are the same as in the inverted bundle configuration and the liquid lead properties are employed for the simulation [16]. An average velocity of $3 \mathrm{~m} / \mathrm{s}$ is maintained to limit corrosion/erosion. Inlet temperature is $650.61 \mathrm{~K}(50 \mathrm{~K}$ above the lead melting temperature), and the maximum cladding temperature was set at $873.15 \mathrm{~K}$ (600 ${ }^{\circ} \mathrm{C}$ ). This temperature limit was applied for the inner and the outer clad surfaces, since both are in contact with liquid lead. Four fuel tubes were used for SCA analyses. The locations of these tubes are shown in Figure 34(b). Neutronics and thermal hydraulics analyses were iterated in a parametric way. The results were used to define the pitch and the fuel tube diameters, which satisfy the temperature limit for $\sim 3 \mathrm{GW}$ fission blanket.

The minimum P/D allowable for the tube is 1.25 based on manufacture and engineering considerations. This prompted the use of different tube diameters with a constant hexagonal pitch for the tube arrangement. The tube diameters toward the target zone are smaller since these tubes have the highest power density. Configuring the fission blanket in this manner results in uneven pressure drop across the fission blanket and a cross-flow. Therefore, CFD analyses are needed to assess the magnitude and subsequent impact of these features.

A CFD model was constructed using STAR-CCM+ to evaluate the accuracy of the SCA model. Two rows of fuel tubes were modeled including the tube with the maximum power density. Symmetric boundary conditions were used since the fission blanket curvature is small compared to that of the fuel tube. The inner and the outer domain boundaries were simulated as straight walls. Figure 36 displays the CFD model. This model is adequate for initial comparison with the obtained SCA results. In all plots, the increasing $x$-coordinate corresponds to increasing radial distance from the target, and the positive $z$-axis is the primary flow direction. 
Y $\mathrm{Y}$

Figure 36 CFD Geometric model for simulating two rows of fuel tubes. Top and bottom boundaries are symmetry planes; left and right boundaries are adiabatic walls.

The power distributions mapped to the volume mesh cells in STAR-CCM+ using a coordinate table. Power distributions were mapped for both the fuel and the clad regions. The total coolant power is small and it was neglected in the current simulations. A constant mass flow inlet and constant pressure outlet were used in the simulation, which had good convergence behavior. Coolant properties were constant and evaluated at the estimated midpoint temperature. The realizable $k-\varepsilon$ two-layer turbulence model was employed. The fuel was simulated as a solid given its low velocity, which can be regarded as conservative assumption. A polyhedral mesh was generated which was conformal at all material interfaces. The embedded thin mesher was used for the clad, which ensured three cells radially within that region. A prismatic layer was used for all walls in contact with the fluid to provide better turbulence and heat transfer modeling. Figure 37 shows a small cross-section of the mesh. Convergence was achieved within 2000 iterations using a segregated flow solver.
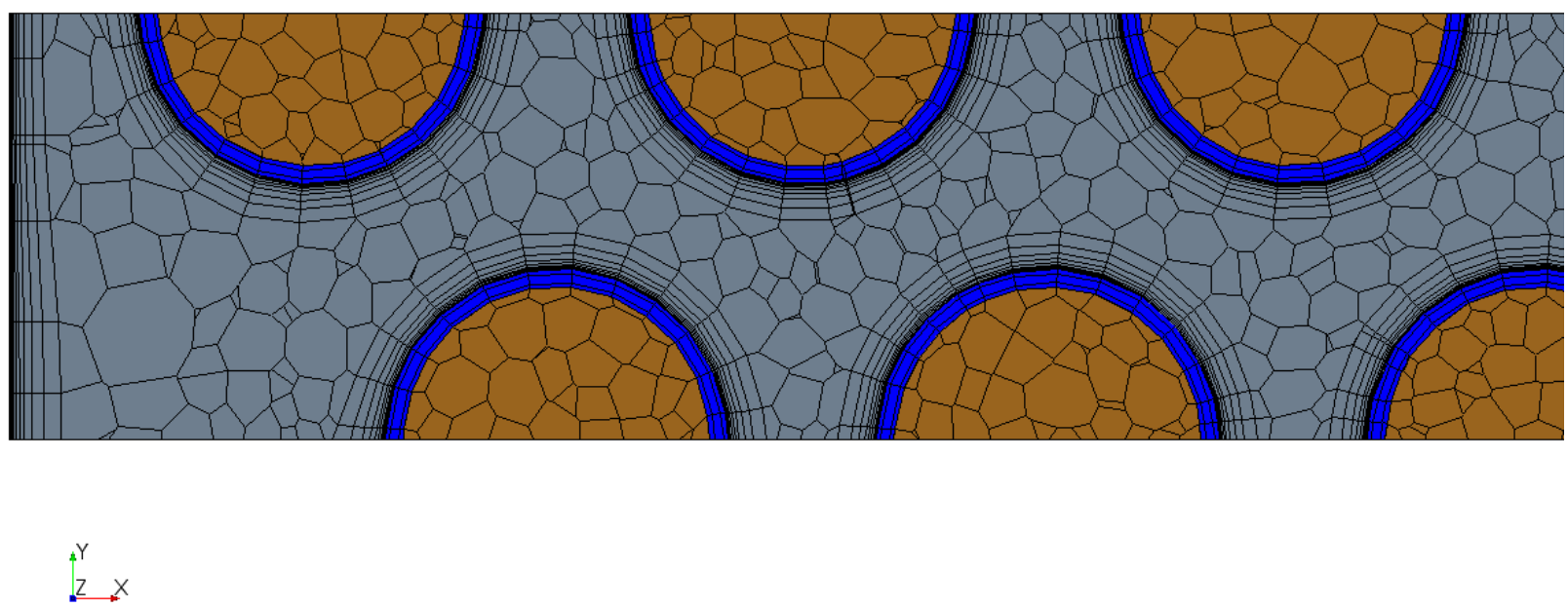

Figure 37 Small section of finite volume mesh used in CFD model, displaying fuel (brown), clad (blue), and coolant (gray) regions.

Extra coolant area near the left and the right boundaries result in higher coolant velocities at these boundaries, as shown in Figure 38. Cross-flows in this geometry are low as shown in Figure 39. In Figures 37 and 38, the left side is that closest to the target. The peak velocity in these locations is above the $3 \mathrm{~m} / \mathrm{s}$ limit, although it should be noted that velocity near the wall is lower. The fuel tubes can slightly adjusted, or an empty spacer tube can be used to reduce the flow area. Otherwise, a baffle or a spacer can mitigate this issue by mixing and distributing flow more evenly. 


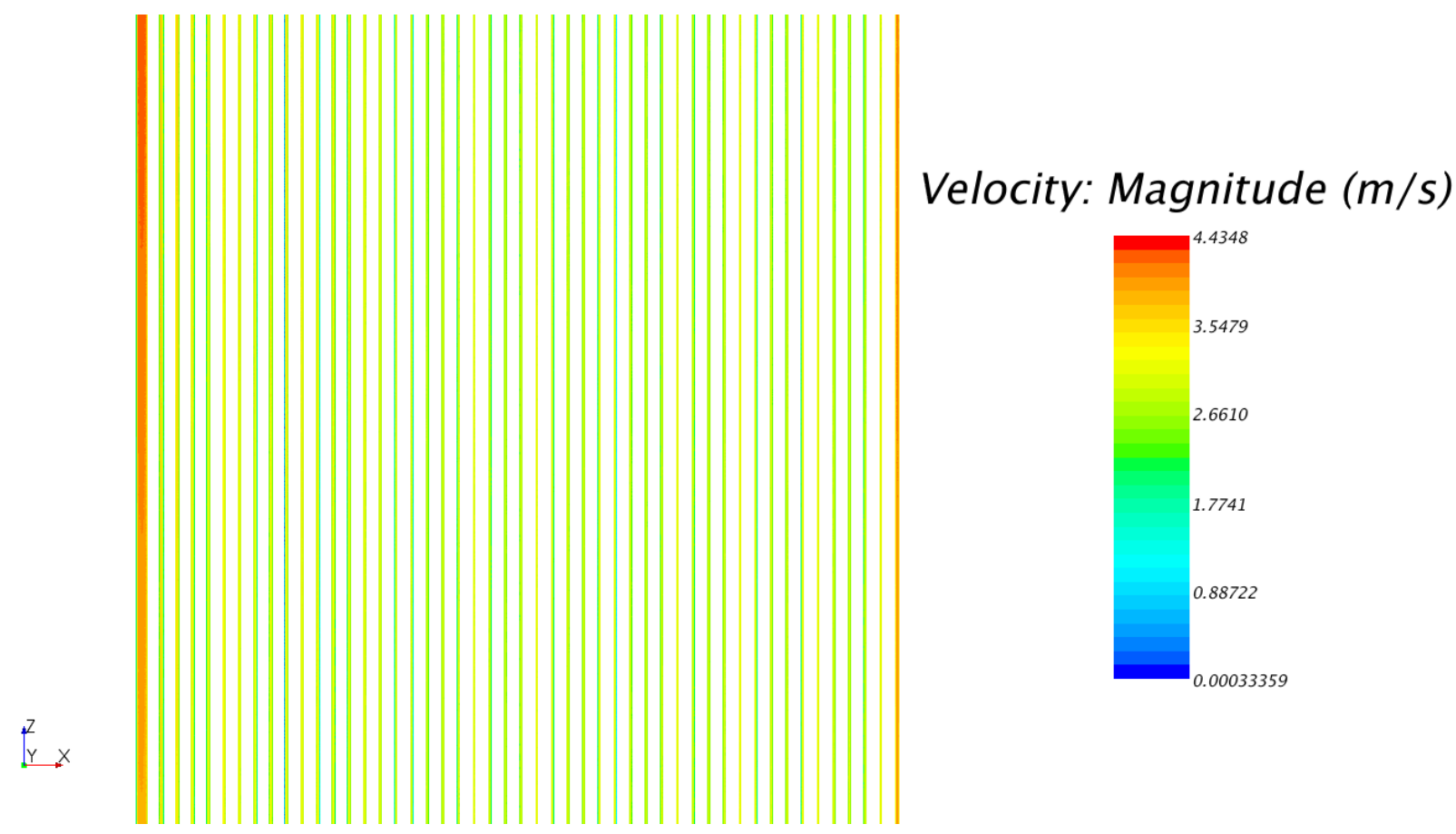

Figure 38 Zoomed view of velocity magnitude on an axial symmetry plane.

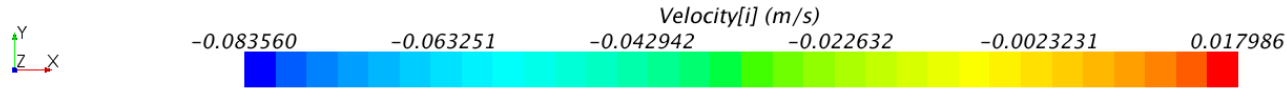

Figure 39 Radial component of velocity distribution.

The peak temperatures are about $11 \mathrm{~K}$ higher than the CFD-SCA results. Since other approximations are conservative (no axial conduction, etc.) then the Westinghouse correlation is conservative for P/D outside its valid range [23]. Figure 40 shows the cladding temperature distribution and the region size with temperature above $873 \mathrm{~K}$ is small. It is located near the axial center of the first few fuel tubes near the target as shown in Figure 41. The maximum cladding surface temperature as a function of the fuel tube position is shown in Figure 42 and it was used to adjust the rod diameters and locations for an updated fission blanket design. Small changes results in a temperature reduction to the desired levels. 


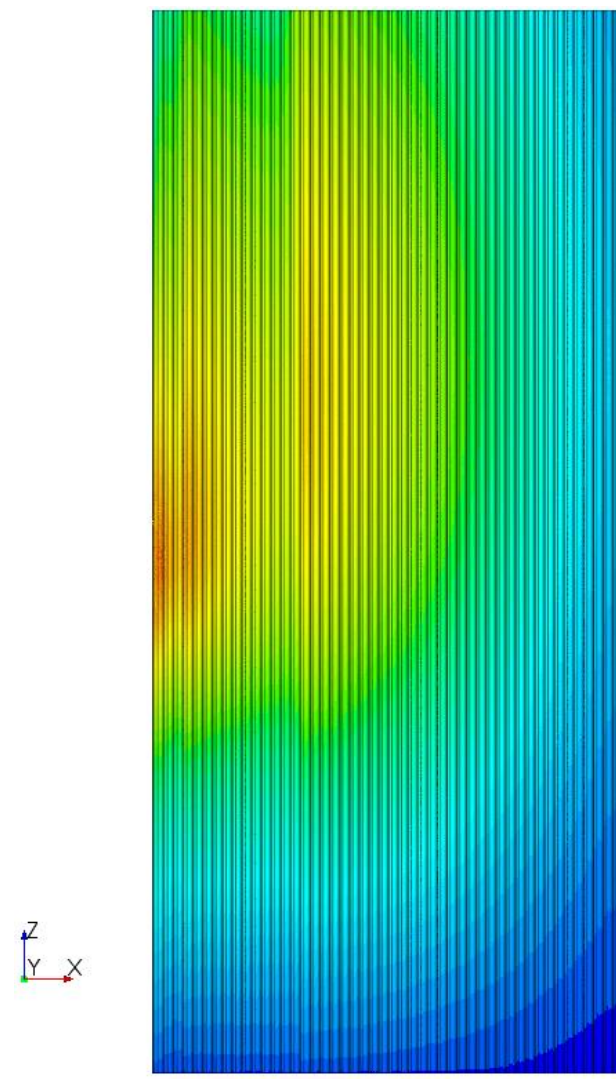

Temperature $(K)$

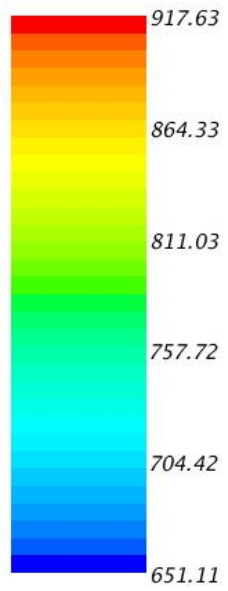

Figure 40 Cladding inner surface radial temperature distribution.

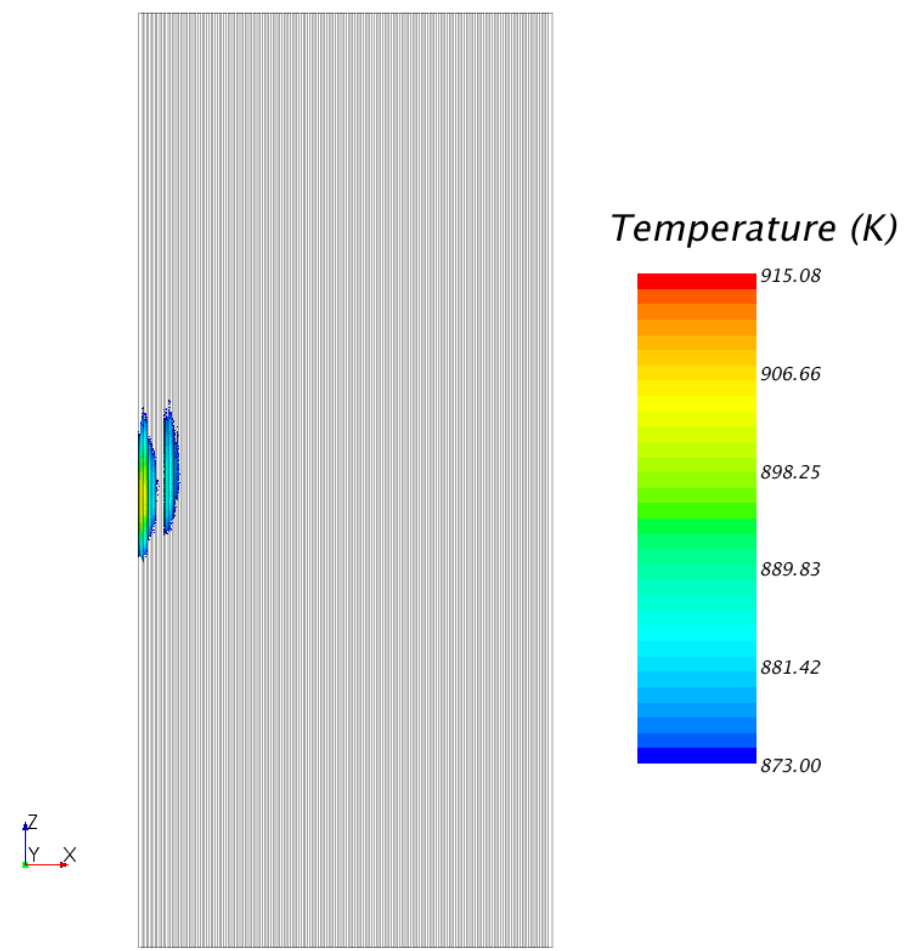

Figure 41 Cladding inner surface radial temperature distribution exceeding the $873 \mathrm{~K}$ temperature limit. 


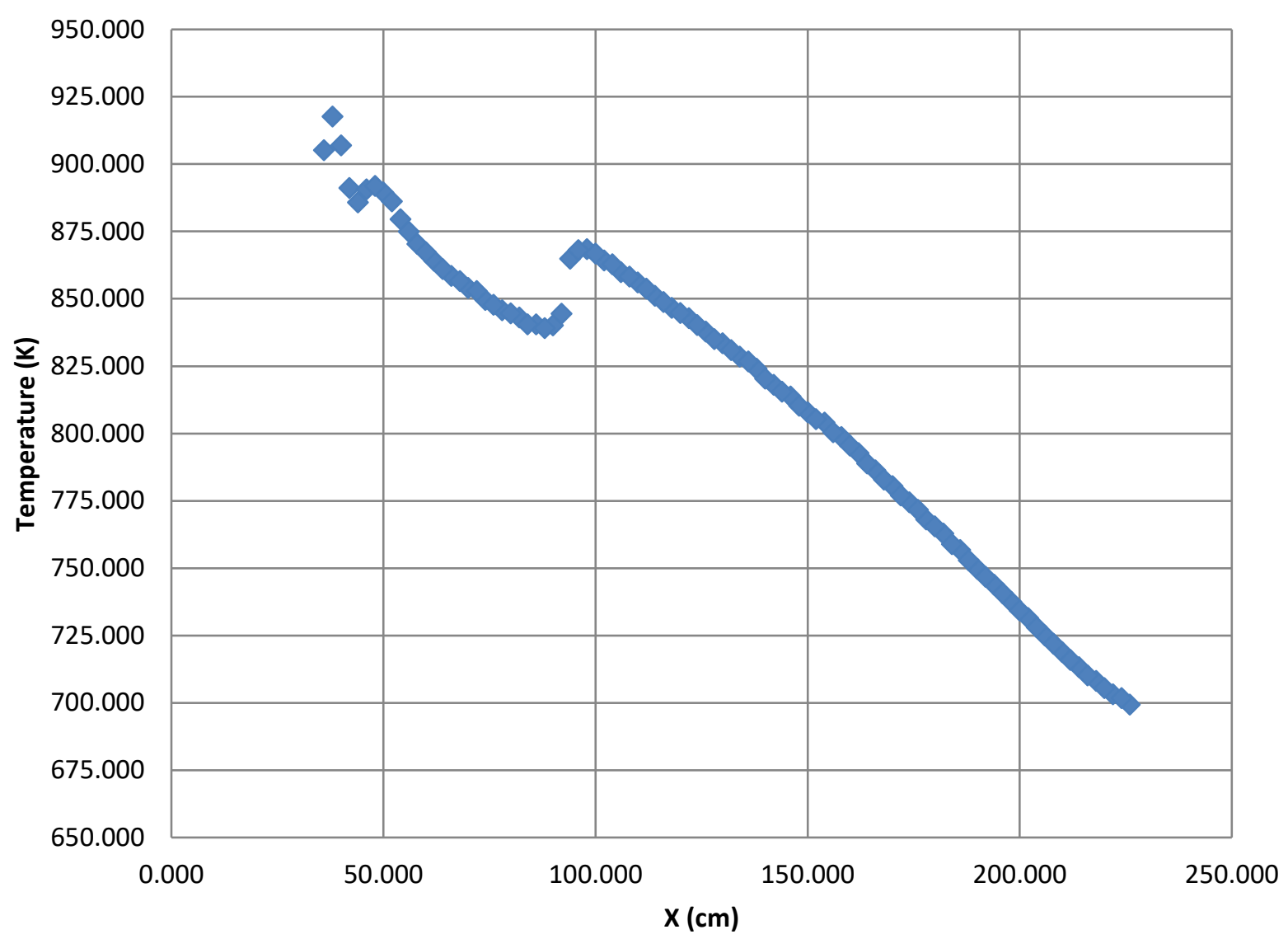

Figure 42 Maximum clad surface temperature as a function of the fuel tube center coordinate. Temperature jump upward at $\sim 100 \mathrm{~cm}$ is due to the fuel tube diameter change.

An updated fission blanket configuration was meshed and STAR-CCM+ was used to obtain an updated temperature distribution. Figures 43 and 44 show the area with temperature exceeding the temperature limit was significantly reduced. In addition, the peak temperature was reduced by about $20 \mathrm{~K}$ and a smoother temperature transition between the fuel tubes with different diameters.

A final design change reduces the number of fission blanket loops to six. This decreases the number of pumps and the required manifolds. In addition, six sectors arrangement matches the hexagonal pitch arrangement of the fuel tubes as shown in Figure 45. New geometrical models were developed to assess the peak temperature of the fuel tubes and to investigate the effect of the sector boundaries on the flow and thermal behavior. The eleven tube rows closest to the target were simulated in full, with a symmetric boundary condition at the model end furthest from the target. This model captures the variety of spaces near the fuel tubes at both the inner and the lateral walls, and the transitions between different fuel tube diameters. It is focused on the inner region of the sector where the power density is highest. A symmetry condition was used the sector mid-plane. 


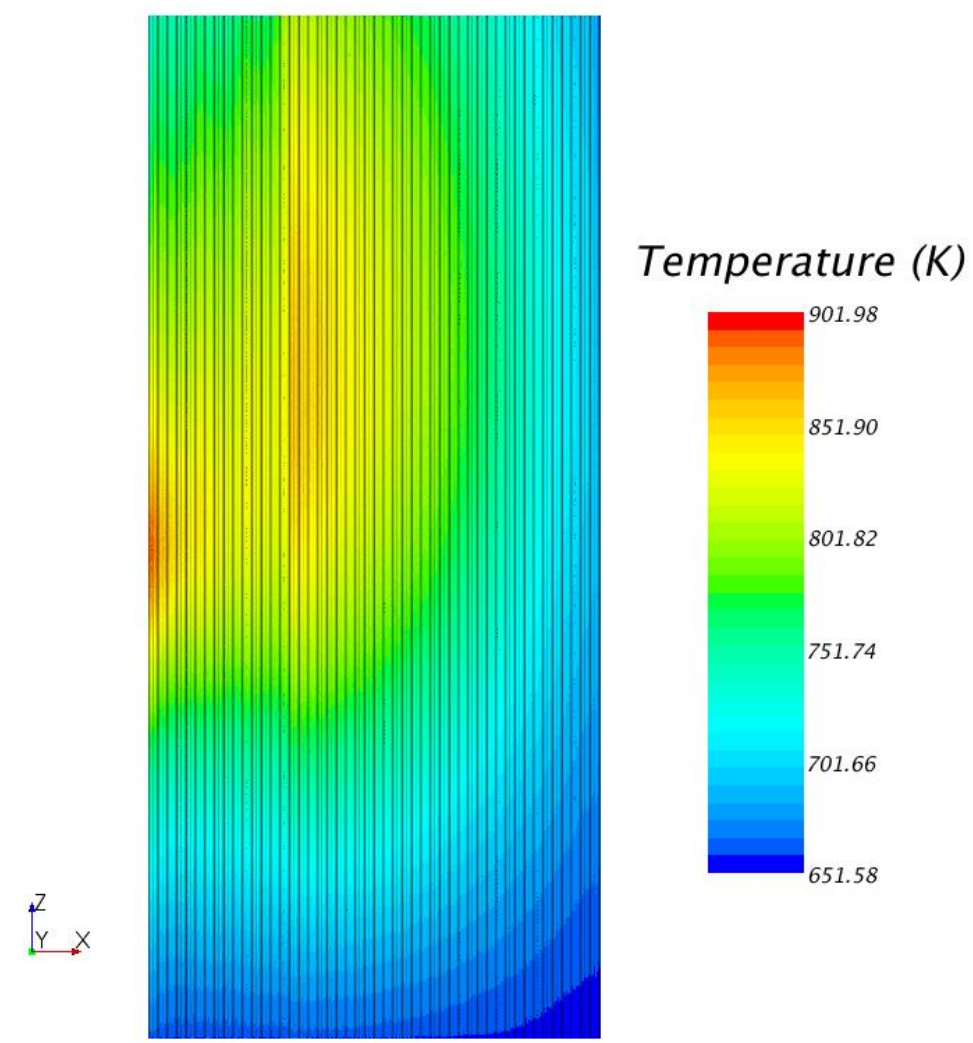

Figure 43 Cladding inner surface radial temperature distribution for the updated fission blanket.

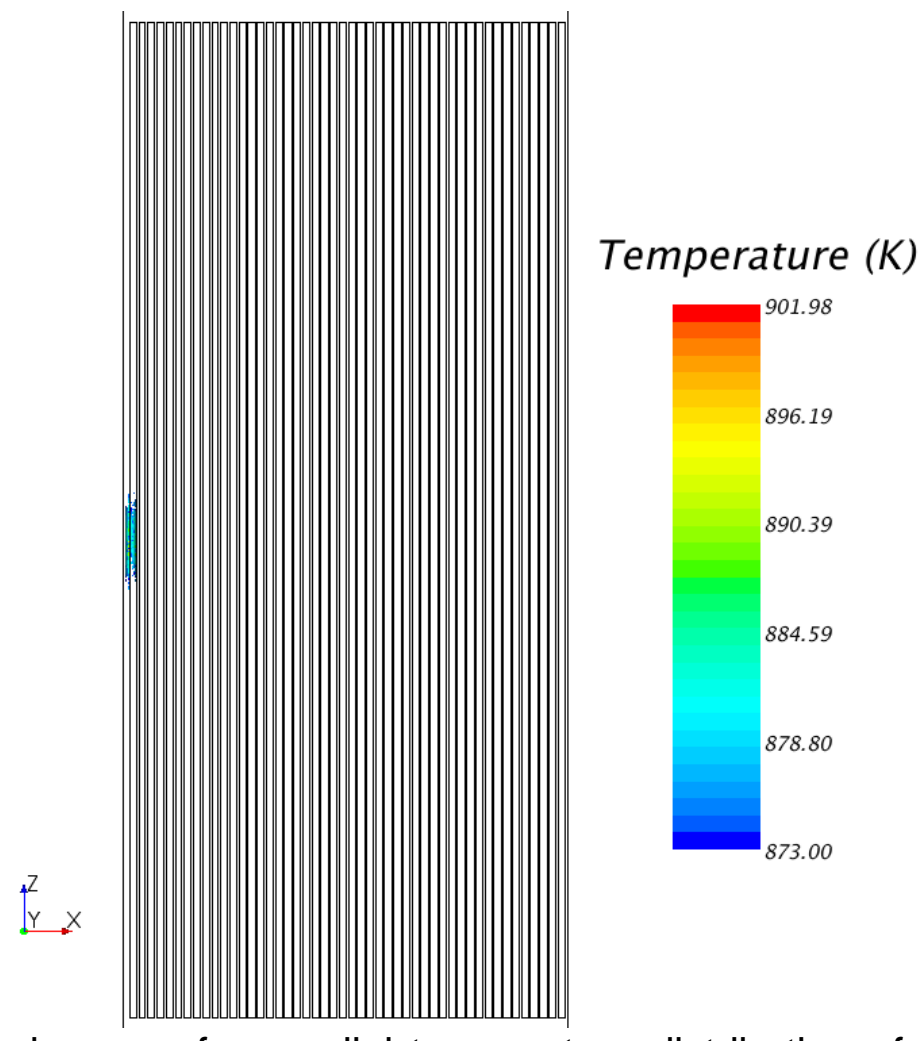

Figure 44 Cladding inner surface radial temperature distribution of the updated fission blanket exceeding the $873 \mathrm{~K}$ temperature limit. 

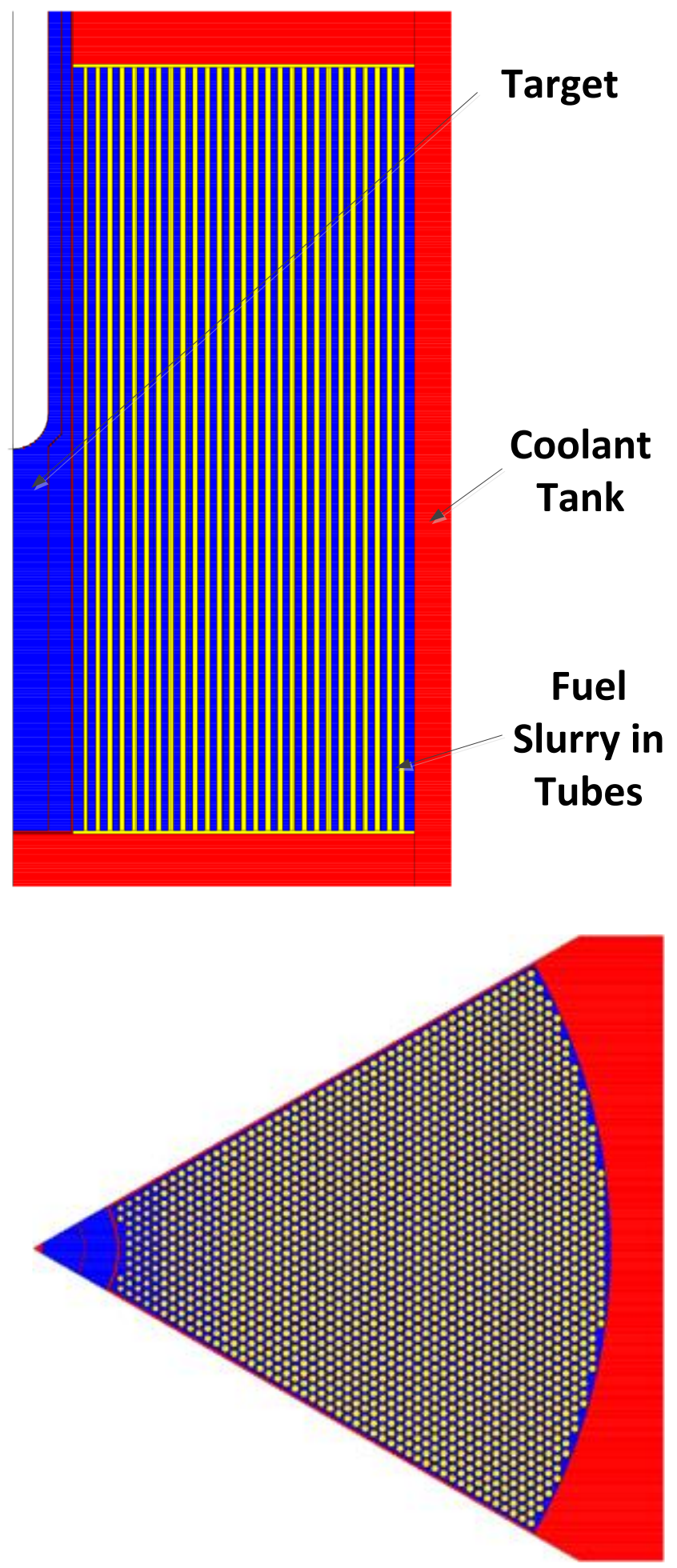

Figure 45 Vertical and mid-plane views of the bundle concept of the fission blanket.

In addition to the inner region model, another strip model consisting of the full radial sector length was also used, similar to that used for the eight-sector configuration described above. This model was updated to include three full radial tube rows instead 
of two as in prior simulations, which gives a better representation of cross-flows between the fuel tubes. Neutronics and thermal-hydraulics iterations continued using the two models to assess the peak temperatures and radial flows.

Figure 46 shows the inner geometrical model, and the mid-plane temperature distribution for the final configuration. The peak power and the peak fuel tube temperature are the nearest to the target zone. The strip model temperatures are shown in Figure 47. The fuel tubes modeled in both models and marked by the gray boxes and have similar temperatures.

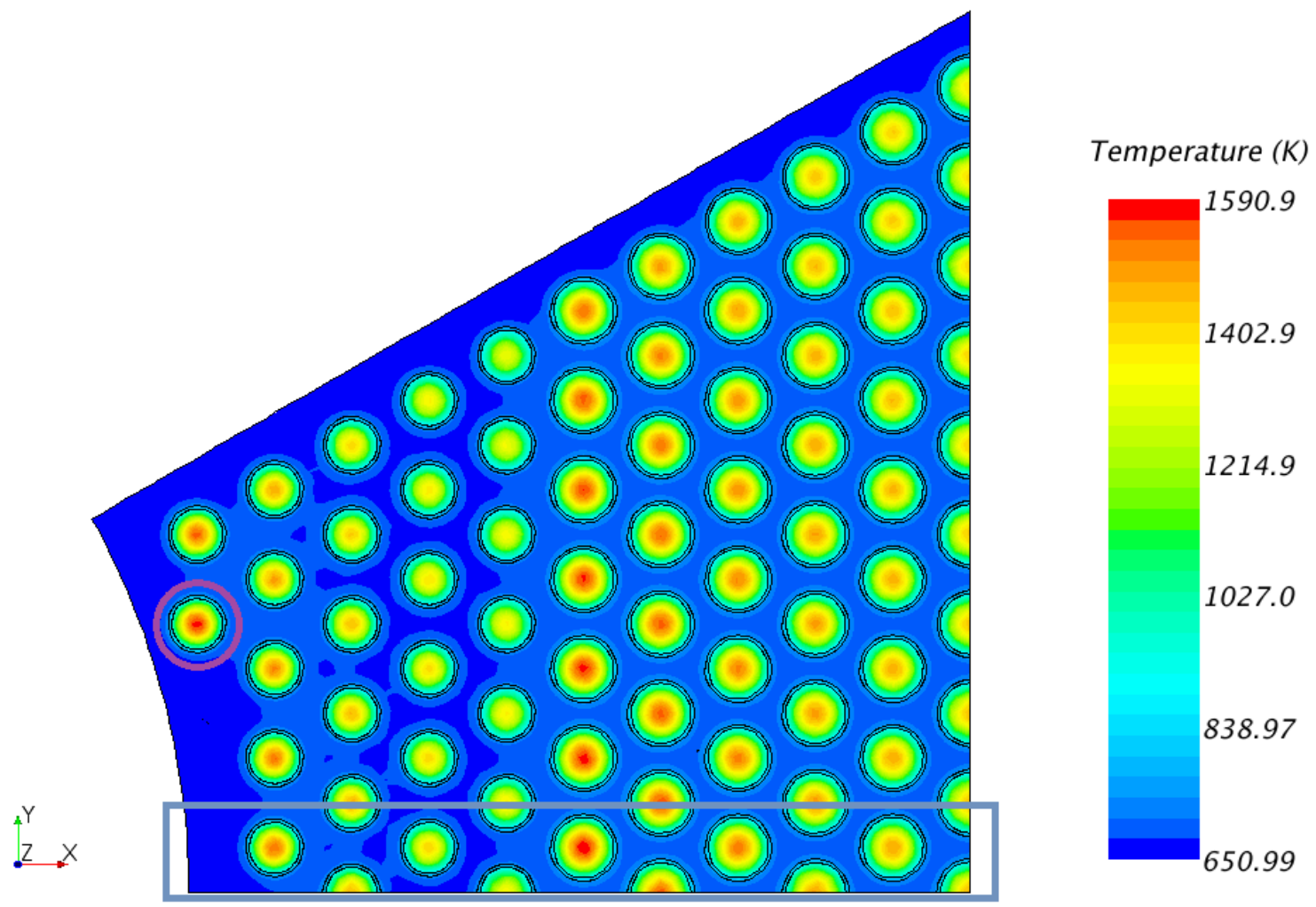

Figure 46 Mid-plane temperature from obtained from the inner model calculation. The purple circle denotes the fuel tube with the peak power/temperature. The gray box contains the fuel tubes used in the strip model.

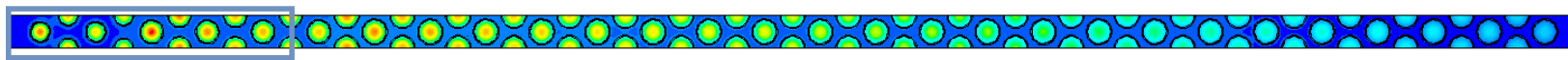

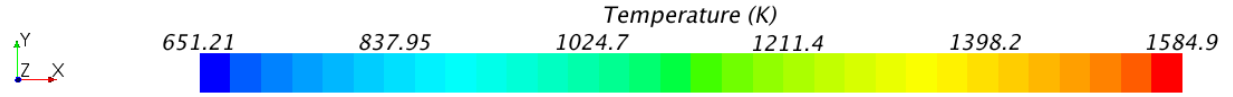

Figure 47 Mid-plane temperature obtained from the strip model calculation. Bottom symmetry plane is coincident with the sector centerline. The gray box contains the tubes used in the inner model. 
The final six-sector configuration has a number of advantages over that of the previous eight-sector configuration. Table XI compares some key temperature values of both configurations. The final configuration reduces the peak surface temperature by $\sim 15$ $\mathrm{K}$. This brings the peak surface temperature within an acceptable range of the design limit. In addition, it reduces significantly the pressure drop. For all of these reasons, the six-sector tube configuration was selected for the final fission blanket configuration.

Table XI Comparison of key thermo-fluid system performance parameters for the eightsector and six-sector cases.

\begin{tabular}{|c|c|c|}
\hline Parameter & Eight-Sectors & Six-Sectors \\
\hline $\begin{array}{c}\text { Max Fuel Tube Surface } \\
\text { Temperature (K) }\end{array}$ & 902.0 & 885.4 \\
\hline $\begin{array}{c}\text { Average Lead Outlet } \\
\text { Temperature (K) }\end{array}$ & 743.7 & 742.4 \\
\hline Frictional Pressure Drop (kPa) & 122.7 & 101.9 \\
\hline
\end{tabular}

The current configuration has some extra space between the inner vessel walls and the fuel tubes. This results in extra flow velocity in these locations, which is above the 3.0 $\mathrm{m} / \mathrm{s}$ velocity limit. This improves the cooling of the fuel tubes near these spaces. A hypothetical arrangement of dummy (spacers) tubes was investigated to see if it produced any significant effect on the fuel tube temperatures. The dummy tubes were placed as if the tube lattice pitch simply continued to the flow boundary as shown in Figure 48. This arrangement is to illustrate the basic idea for controlling the flow.

The differences between the velocity fields of the "spaces" and "no-spaces" cases can be seen in Figure 48. These tubes can be seen to provide a more even flow-field in the front section of the sector and the average velocity changes at the fuel tube diameter change. The peak velocity is reduced. This hypothetical arrangement increases the peak surface temperature of the fuel tubes by less than $2 \mathrm{~K}$. For this reason, the effect of the additional space can be ignored with respect to the peak temperature of the fuel tubes.

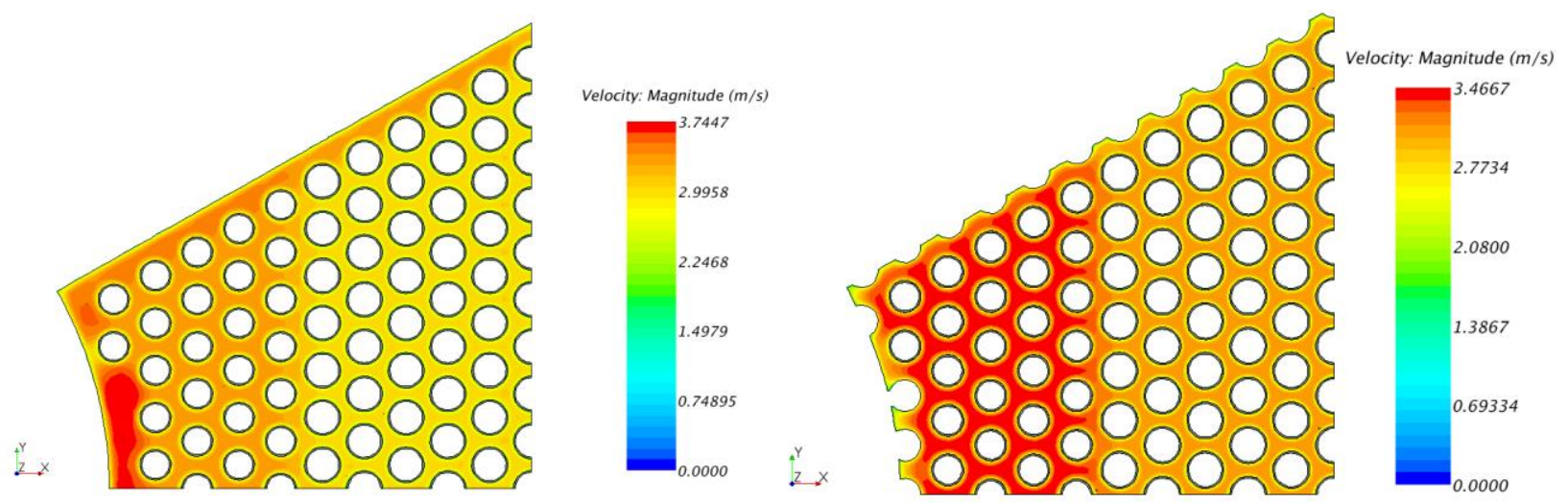

Figure 48 Comparison of mid-plane velocity distribution for the selected configuration (left) and for the same configuration with dummy tubes (right). 
The $3 \mathrm{~m} / \mathrm{s}$ average inlet coolant velocity was used for the entire sector for all simulations. This ensures the lowest temperatures for the fuel tubes, but it is not the optimal choice for the system. It is desirable to reduce the flow as much as possible in the areas with less power density. This increases the average outlet temperature of the coolant, which is beneficial for thermal efficiency of the system. Moreover, decreasing the flow rate reduces the frictional pressure drop, in turn reducing the required pumping power.

A few simulations were examined with inlet boundary conditions representing hypothetical orifice conditions based on the radial distance from the target. These analyses do not include spacer grids, tube sheets, or any other potential structural materials in the fission blanket. Three different simulations were examined: 3 velocity zones, 4 velocity zones, and variable velocity zone as shown in Figure 49 . The first two simulations have constant velocities over equal portions of the radial geometrical model. The last simulation has a velocity of $3 \mathrm{~m} / \mathrm{s}$ near the target and it is reduced as a function of the radial distance according to the next equation,

$$
V=4.985337-1.910406 * R
$$

$V$ is the velocity and $R$ is the radius. These different inlet distributions were examined using the strip model of the final tube configuration.

The obtained results shows that the proper orifice design or flow zoning can have a large impact on the thermal performance of the fission blanket. This shows that the constant $3 \mathrm{~m} / \mathrm{s}$ average inlet velocity results in the lowest blanket thermal efficiency. The variable velocity profile can yield substantial increases in outlet temperature, 19-36\% higher than the constant inlet velocity as shown in Table XII. The 4-velocity zones and the variable velocity provide the largest gains. In addition, as expected, the pressure drop is reduced by 26 to $36 \%$ as shown in Table XII. These benefits come with only small increases in the peak surface temperature.

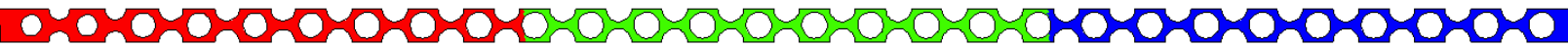

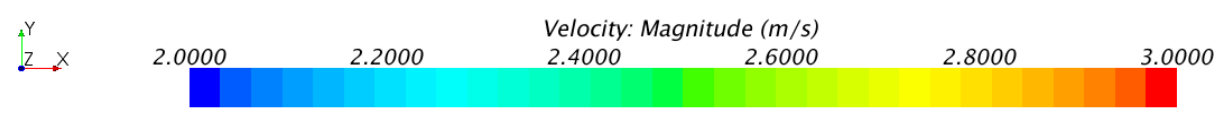

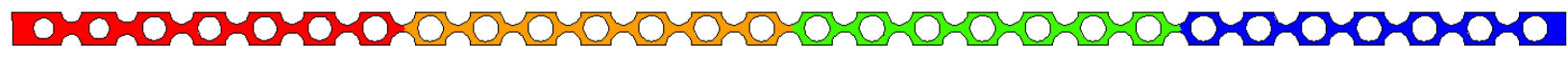
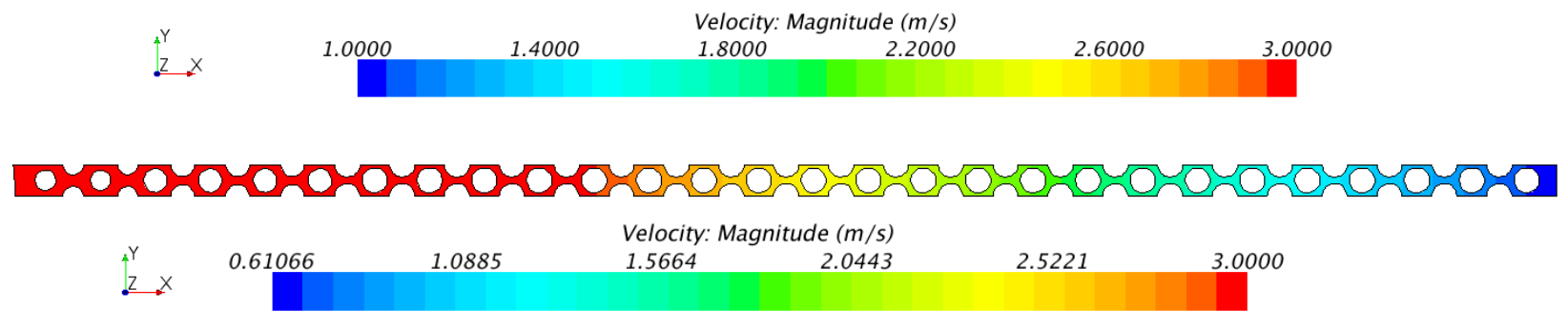

Figure 49 Inlet velocity distributions for the 3-zone (top), 4-zone (middle), and line (bottom) cases. 
Table XII Thermal-hydraulic performance comparison for various inlet velocity profiles.

\begin{tabular}{|l|c|c|c|c|}
\hline \multicolumn{1}{|c|}{ Parameter } & Base Case & 3-Zone & 4-Zone & $\begin{array}{c}\text { Linear } \\
\text { Change }\end{array}$ \\
\hline Max Tube Surface Temperature (K) & 876.9 & 880.5 & 894.6 & 888.4 \\
\hline Average Fluid Outlet Temperature (K) & 742.4 & 759.7 & 775.0 & 772.8 \\
\hline Frictional Pressure Drop (kPa) & 101.9 & 74.9 & 64.9 & 67.5 \\
\hline
\end{tabular}

The final configuration was simulated with a full half-symmetric model of the bundle sector. This can validate the two-model approach used above, and it removes any concerns regarding the boundary conditions. This simulation increases significantly the size and the complexity of the model, so it can only be used for the final configuration. This model contains more than 900 tubes, and the computational mesh size is roughly 136 million cells.

The computational methods used were similar to those employed for the bundle models described before. One difference was for the power density mapping, power densities were tallied radially for each cell (fuel, clad, and coolant materials) over the total height of the fission blanket. Then the axial distribution was calculated by MCNP as a function of the radius for generating the detailed power density distribution for the thermal hydraulics analyses. The results are three power distributions for the fuel, clad, and coolant materials. A script was written to perform nearest-neighbor interpolation to map each power distribution to the STAR-CCM+ mesh cells located within the hexagonal boundary of a given MCNP lattice cell. The power distributions are shown in Figures 50 and 51. The fuel power density is more than one order of magnitude higher than the corresponding values for the coolant and the clad.

Temperature and velocity distributions at various locations are shown in Figures 52 to 56 . The peak surface temperature of the fuel tubes is $871 \mathrm{~K}$, so all tubes are within the design limit. Figure 52 shows the three fuel tubes with surface temperatures above $870 \mathrm{~K}$. The pressure drop in the fission blanket is $113 \mathrm{kPa}$ and the coolant outlet temperature is $730 \mathrm{~K}$. The temperature distribution of the outlet coolant is uneven. However, a more uniform outlet coolant temperature distribution can be obtained by using tube sheets/grids and orifice techniques as examined before. Figures 53 and 54 show the fuel tubes with the highest temperatures are near the tube diameter transitions. Again, a small area of the tube surface is above the limit by $1 \mathrm{~K}$. The velocity distribution is relatively flat for the bundle as shown in Figure 56, with elevated values higher than $3 \mathrm{~m} / \mathrm{s}$ in the open spaces near the inner and outer walls. This can be changed with dummy cylinders or some similar techniques as examined before without changing the temperatures of the surrounding fuel tubes. Based on the obtained results, this configuration satisfies the design requirements. 


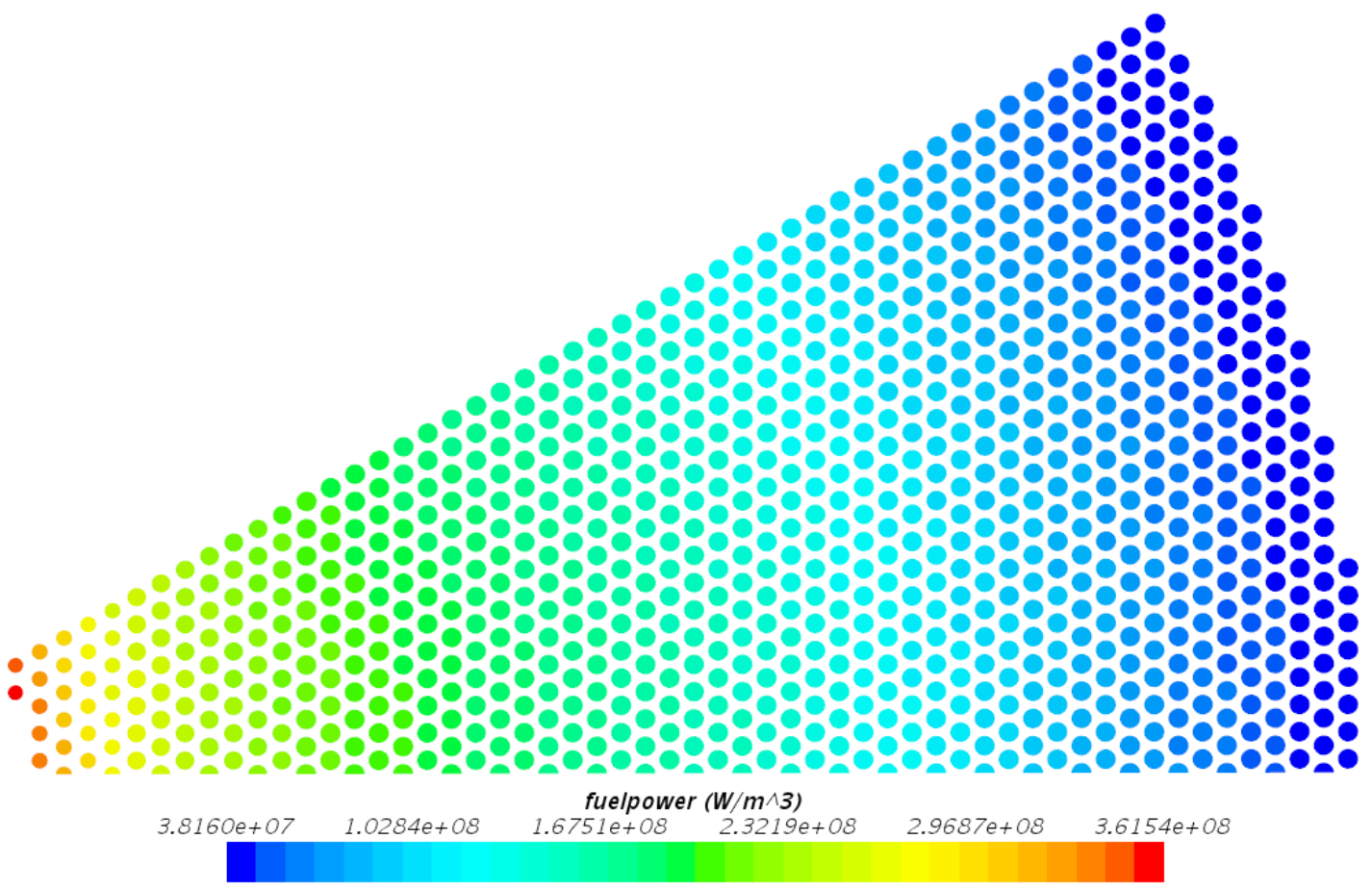

(a) Fuel Material

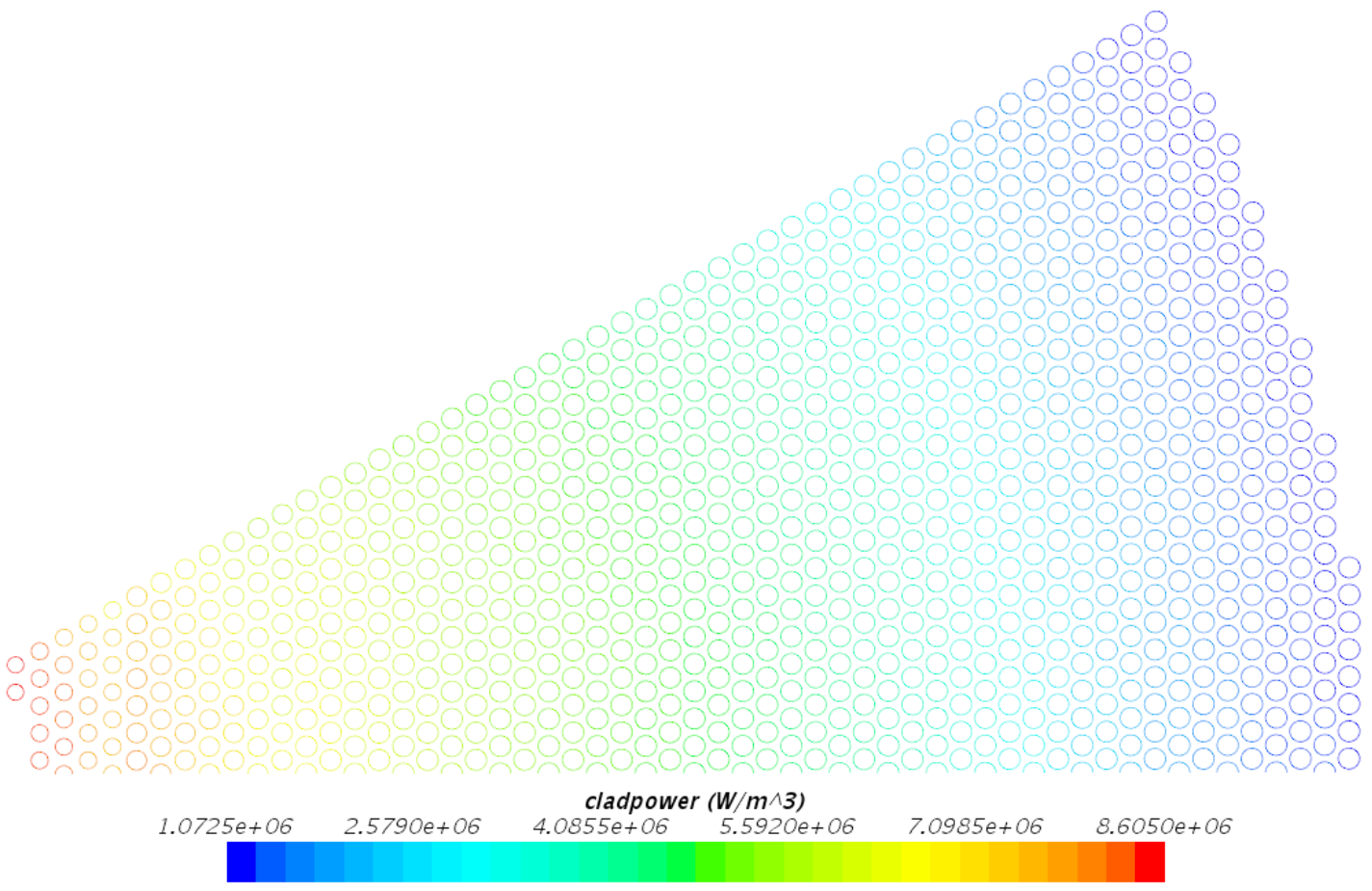

(b) Clad Material 

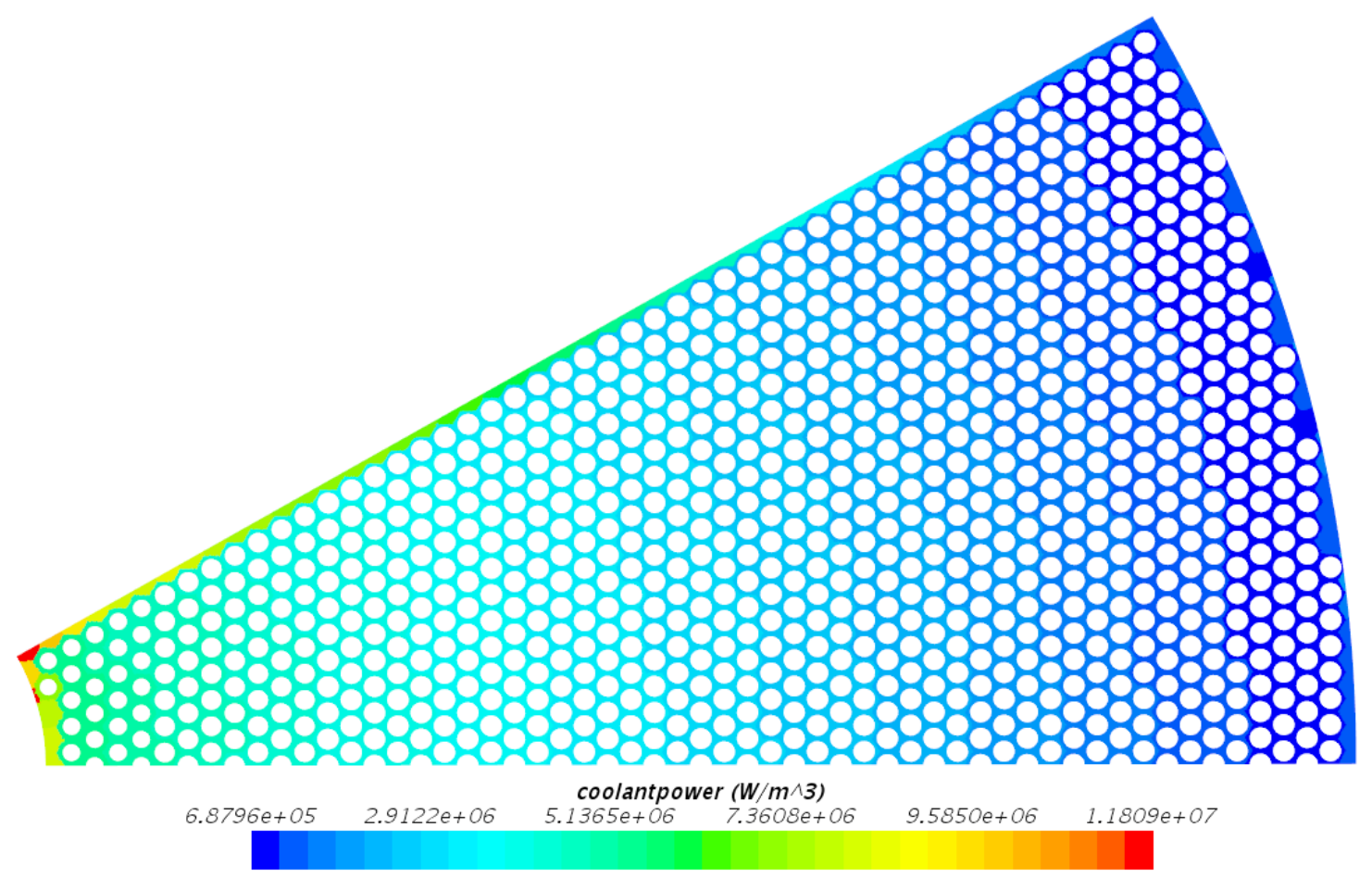

(C) Coolant Material

Figure 50 Power distribution map at the mid-plane.

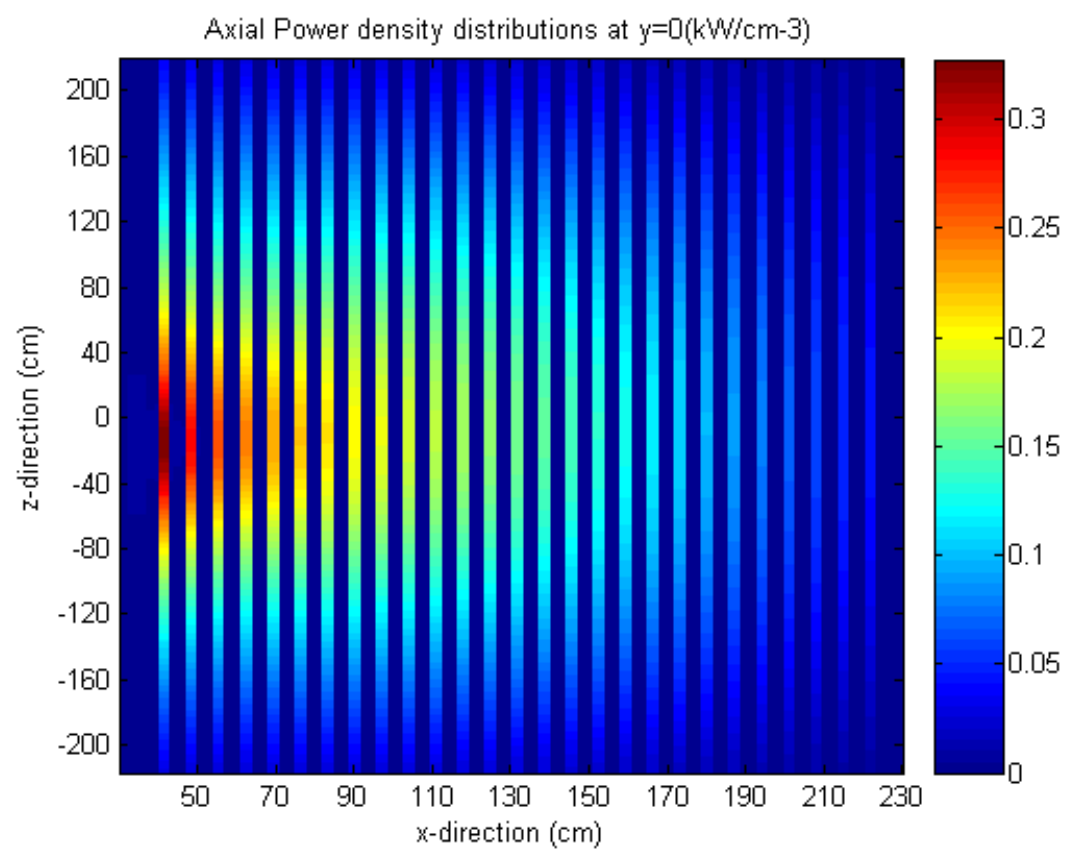

Figure 51 Axial fuel power distribution at the symmetry plane 

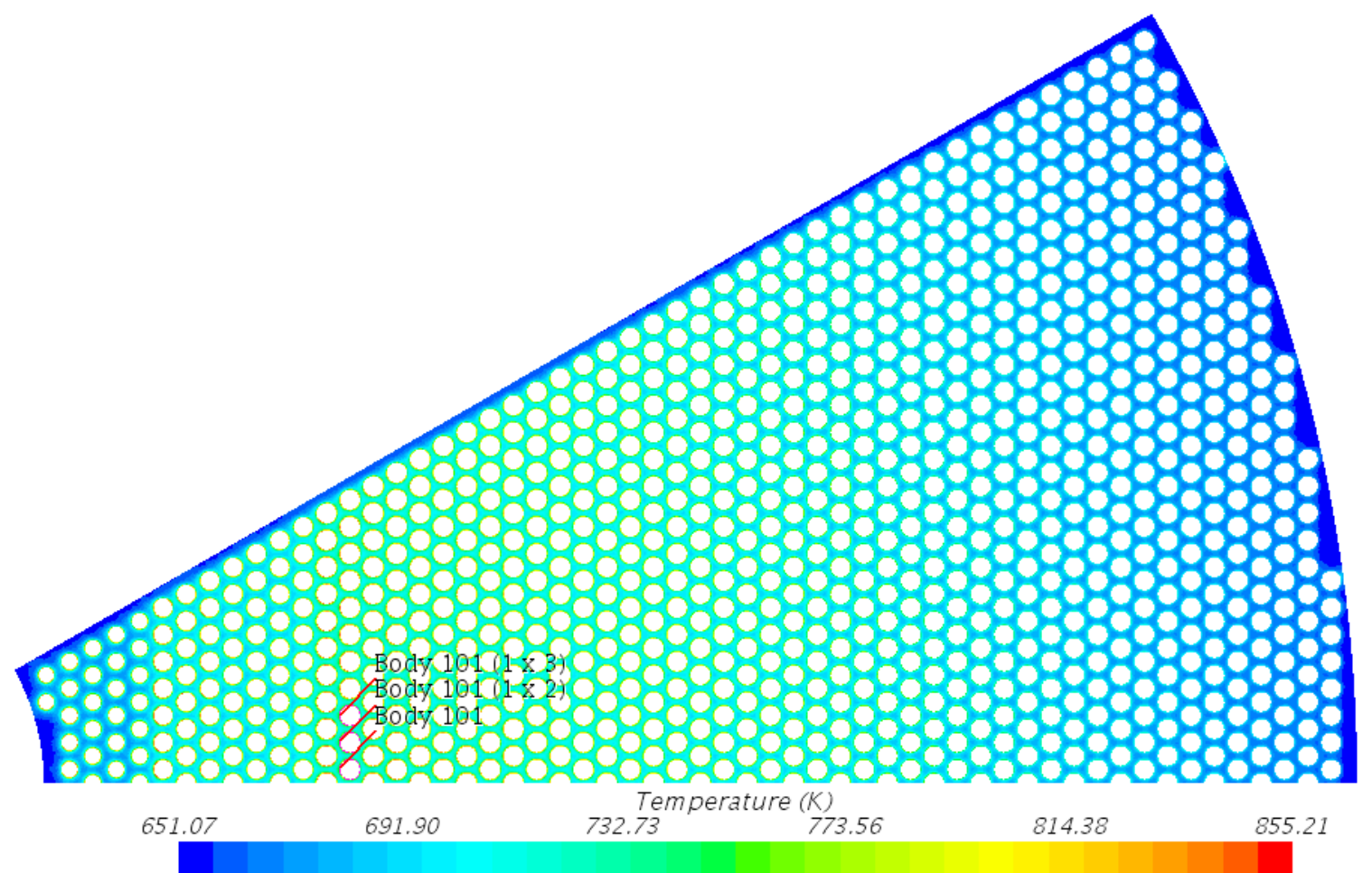

Figure 52 Mid-plane temperature distribution for the coolant and cladding, highlighting the tubes with surface temperature above $870 \mathrm{~K}$.

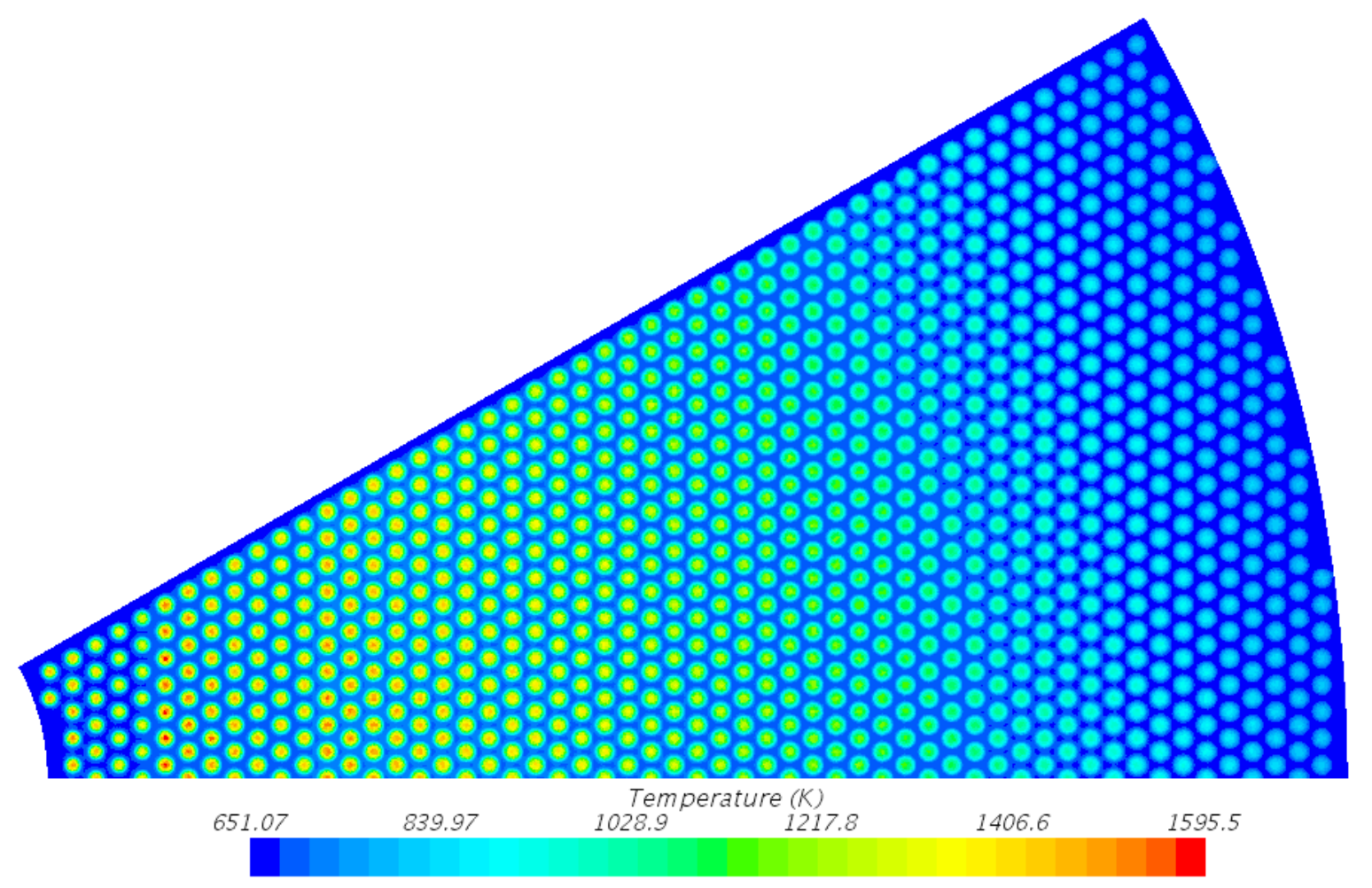

Figure 53 Mid-plane temperature distribution of all materials. 


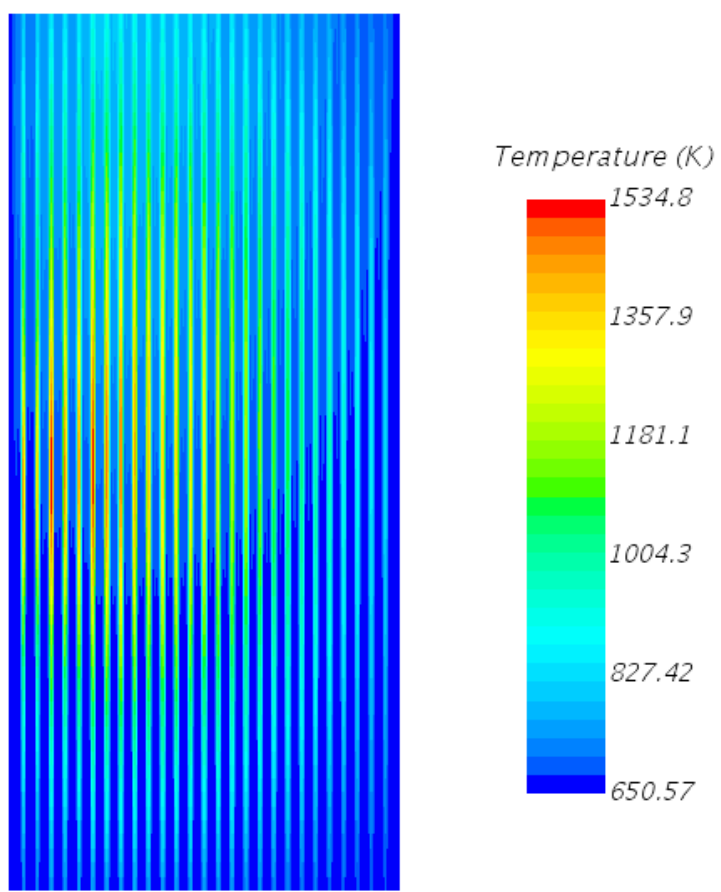

Figure 54 Axial temperature distribution at the symmetry plane of all materials 

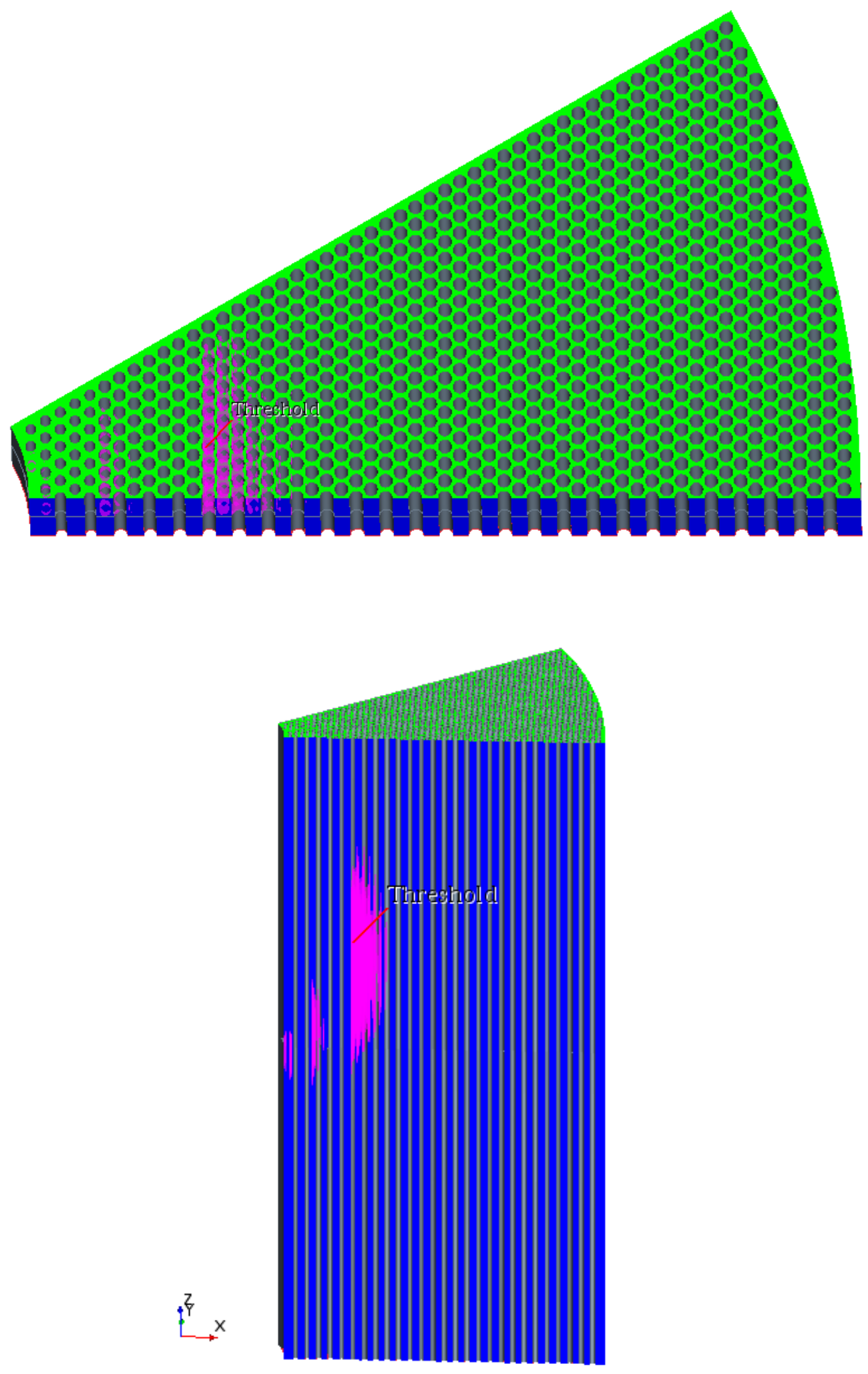

Figure 55 Tube surface temperatures above 850K are highlighted with pink. These tubes are located near the tube diameter transitions. 


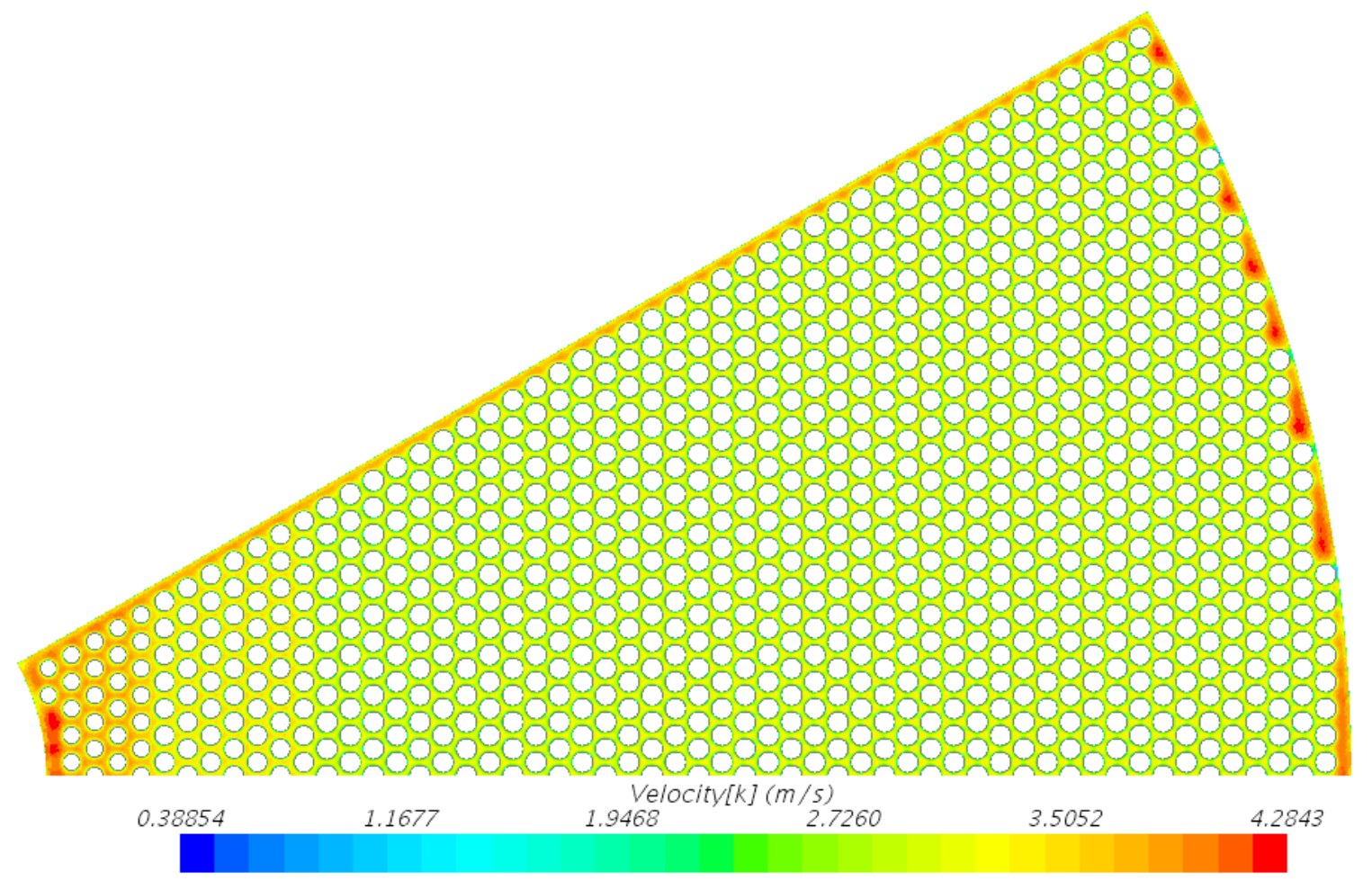

Figure 56 Axial velocity profile at the mid-plane.

Table XIII lists the system parameters obtained from the analysis. Compared with previous inverted bundle model, the subcritical assembly tank of the bundle model is slightly larger in order to accommodate more coolant volume. The actinide fuel volume utilized is slightly increased in this configuration. More importantly, in this design, the bundle tube size is much larger. The total number of tubes inside the subcritical assembly is also reduced by $\sim 40 \%$. Due to the loss of the source neutrons in the tubes and coolant, the total power generated in the fission blanket is slightly smaller than that from the homogeneous case.

\subsection{Reflector Analyses}

The graphite reflector was utilized in the preliminary design to improve the neutron economy in the subcritical fission blanket since it is an excellent neutron moderator. Neutrons leaking out of the subcritical fission blanket are thermalized in the graphite reflector and a fraction of them reflected back to the fission blanket. These reflected thermal neutrons generate a high fission rate at the fission blanket boundary causing a high power peak as shown in Figure 57. The use of the stainless steel or the ferritic steel (HT-9) as a reflector material eliminates this power peak as shown in Figures 22, 23, 50, and 51 . 
Table XIII. Main parameters of the conceptual design using the bundle model.

\begin{tabular}{|l|c|}
\hline \multicolumn{1}{|c|}{ Parameter } & Value \\
\hline Actinide oxide volume fraction in slurry: & $9 \%$ \\
\hline U/Pu atomic fraction in the fuel: & $47.52 \%$ \\
\hline Target outer radius, cm & 34.27 \\
\hline Subcritical Assembly outer radius, cm & 229.0 \\
\hline Subcritical Assembly height, cm & 440.0 \\
\hline Subcritical Assembly fuel volume, $\mathrm{m}^{3}$ & 31.49 \\
\hline Fuel tube lattice pitch, cm & 4.0 \\
\hline Fuel tube diameters, cm & $2.3-2.9$ \\
\hline Number of fuel tubes & 11142 \\
\hline Fuel volume fraction, ${ }^{3}$ & $44,96 \%$ \\
\hline Effective neutron multiplication factor keff & 0.98 \\
\hline Generated fission power, GW & 2.67 \\
\hline Subcritical Assembly mobile fuel inventory, ton & 27.4 \\
\hline Subcritical Assembly MAs initial inventory, ton & 14.0 \\
\hline
\end{tabular}

Monte Carlo simulations were performed to define the appropriate thickness of the stainless steel reflector. Specifically, the neutron multiplication factor ( $\left.k_{\text {eff }}\right)$ was calculated as function of the reflector thicknesses for the homogeneous concept. As expected, the neutron multiplication factor increases with the reflector thickness and saturates at $\sim 45 \mathrm{~cm}$ as shown in Figure 58. Therefore, all the examined configurations used $50-\mathrm{cm}$ reflector thickness in the radial and axial directions.

Monte Carlo calculations were also performed to study the radiation damage in the subcritical assembly tank, the reflector, and the vessel outside the reflector. With the $25 \mathrm{MW}-1 \mathrm{GeV}$ proton beam, the peak atomic displacement rate in the vessel outside the reflector zone is $6 \mathrm{dpa} / \mathrm{fpy}$. For this life time component, an extra protection is required to reduce the radiation damage, which can be done by adding extra shield materials after the reflector. 


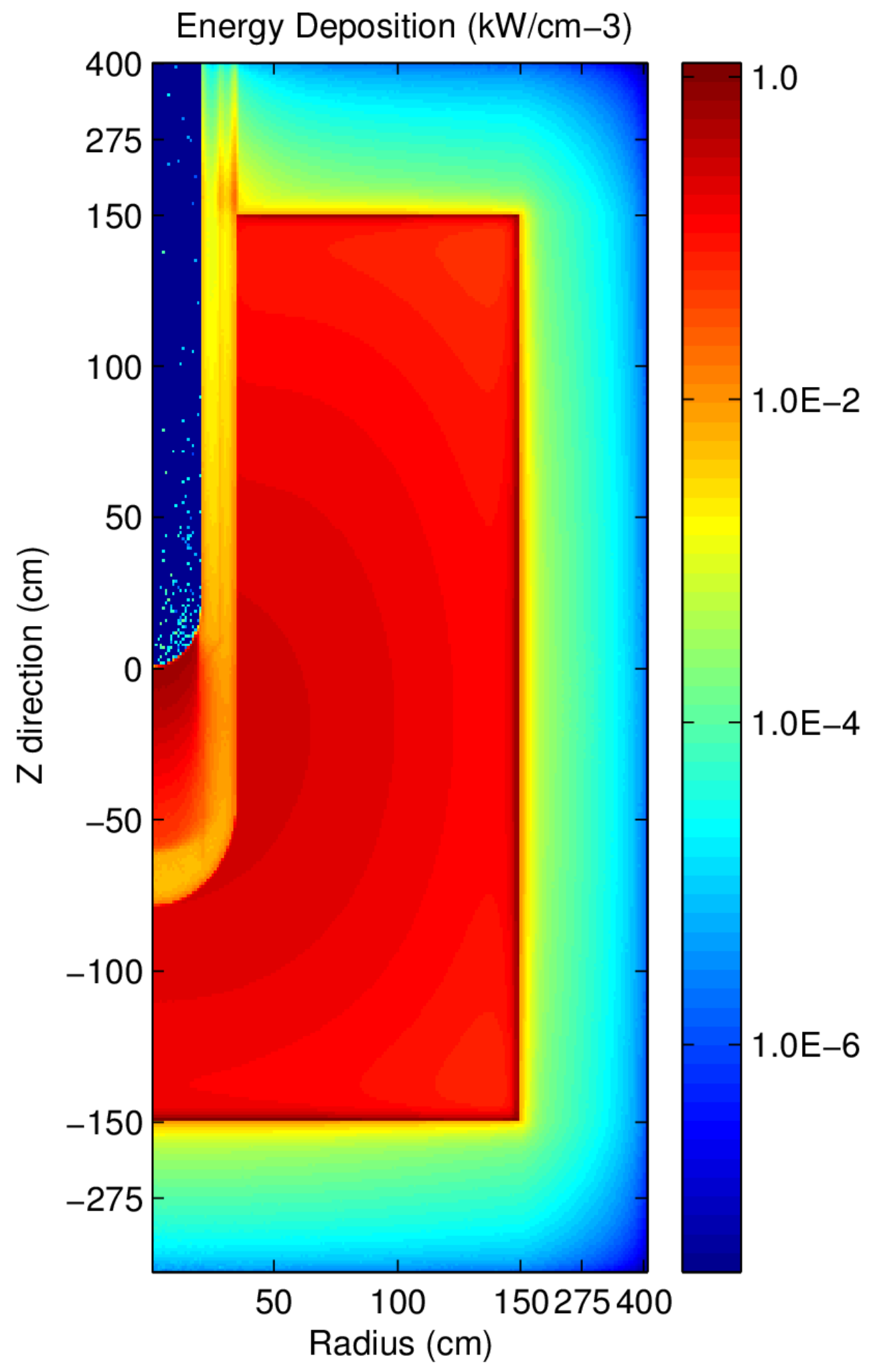

Figure 57 Calculated energy depositions in the homogeneous fission blanket with a graphite reflector. 


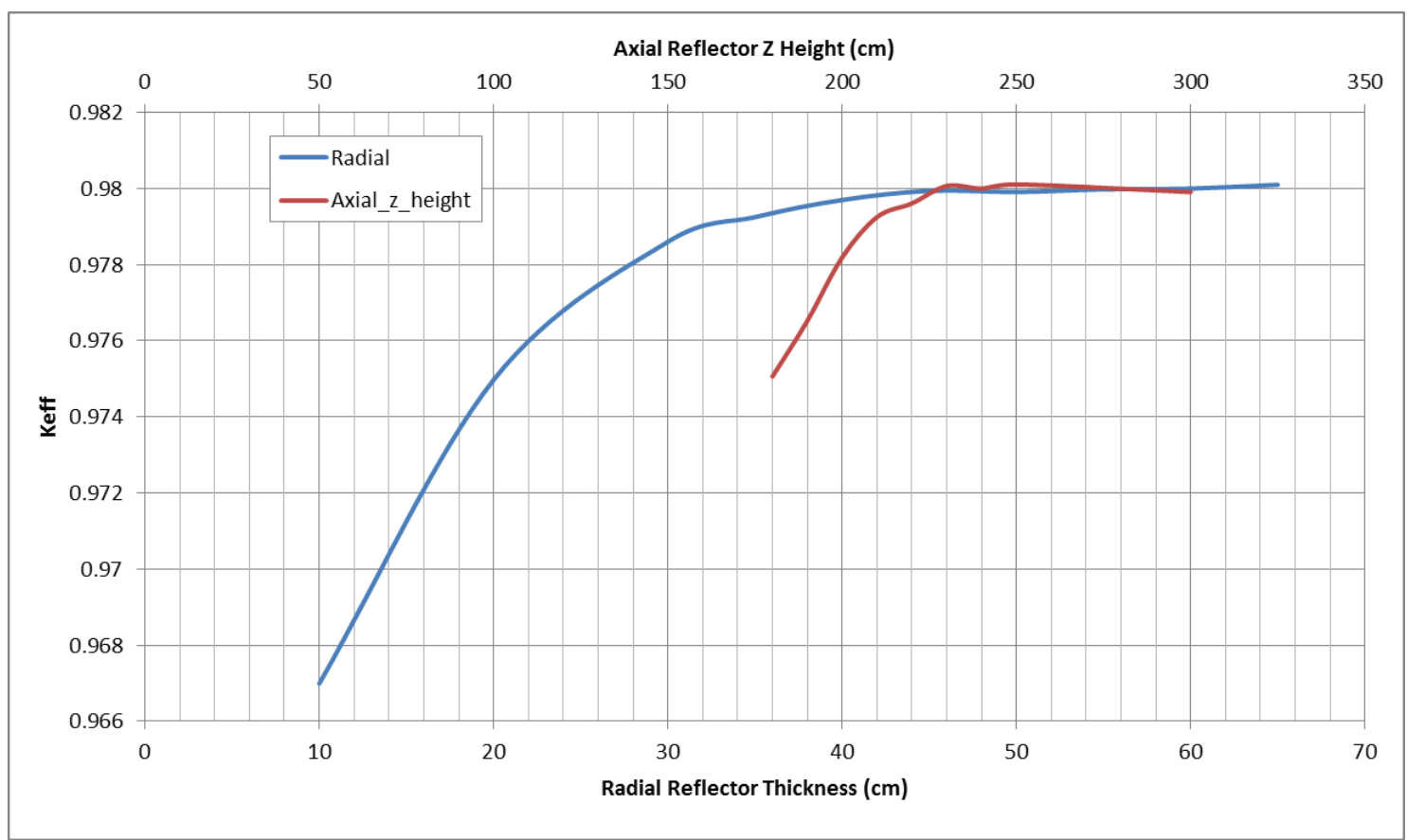

Figure 58 Neutron multiplication factor of the homogeneous concept as function of the reflector thickness in the radial and axial directions.

\section{$5 \quad$ ADS Burnup Analyses}

Burnup analyses were performed to evaluate the performance of the different ADS configurations. The MCNPX code is first utilized to calculate the neutron source generated from the interaction of the $25-\mathrm{MW} 1-\mathrm{GeV}$ proton beam with the liquid lead target at $600 \mathrm{~K}$. The energy spectrum of the generated neutrons are shown in Figure 59. For the volume source, the energy spectrum of the spallation neutrons is recorded at their born site. For the surface source, the spallation neutrons are transported until reaching the target outer surface and their energy spectrum is recorded at their exit from the target surface boundary. In both cases, the neutron parameters are saved for future sampling in the burnup analyses. The energy, the $x$ y $z$ coordinate, the direction, and the time stamp of each neutron are saved in the source file.

The $1 \mathrm{GeV}$ proton beam striking the lead target generates $\sim 15.6$ neutrons per proton from the spallation process. Some high-energy neutrons are generated, $\sim 16 \%$ with energy greater than $20 \mathrm{MeV}$, and $\sim 2 \%$ with energy greater than $200 \mathrm{MeV}$. These highenergy neutrons interact with the lead target material generating more neutrons through $(n, x n)$ reactions. Therefore, the fraction of the neutrons with high energy leaking out of the target surface is significantly reduced. About $1.6 \%$ with energy greater than $20 \mathrm{MeV}$ and only $\sim 0.1 \%$ with energy greater than $200 \mathrm{MeV}$ within the 29.5 neutrons per proton are leaking from the outer target surface. 


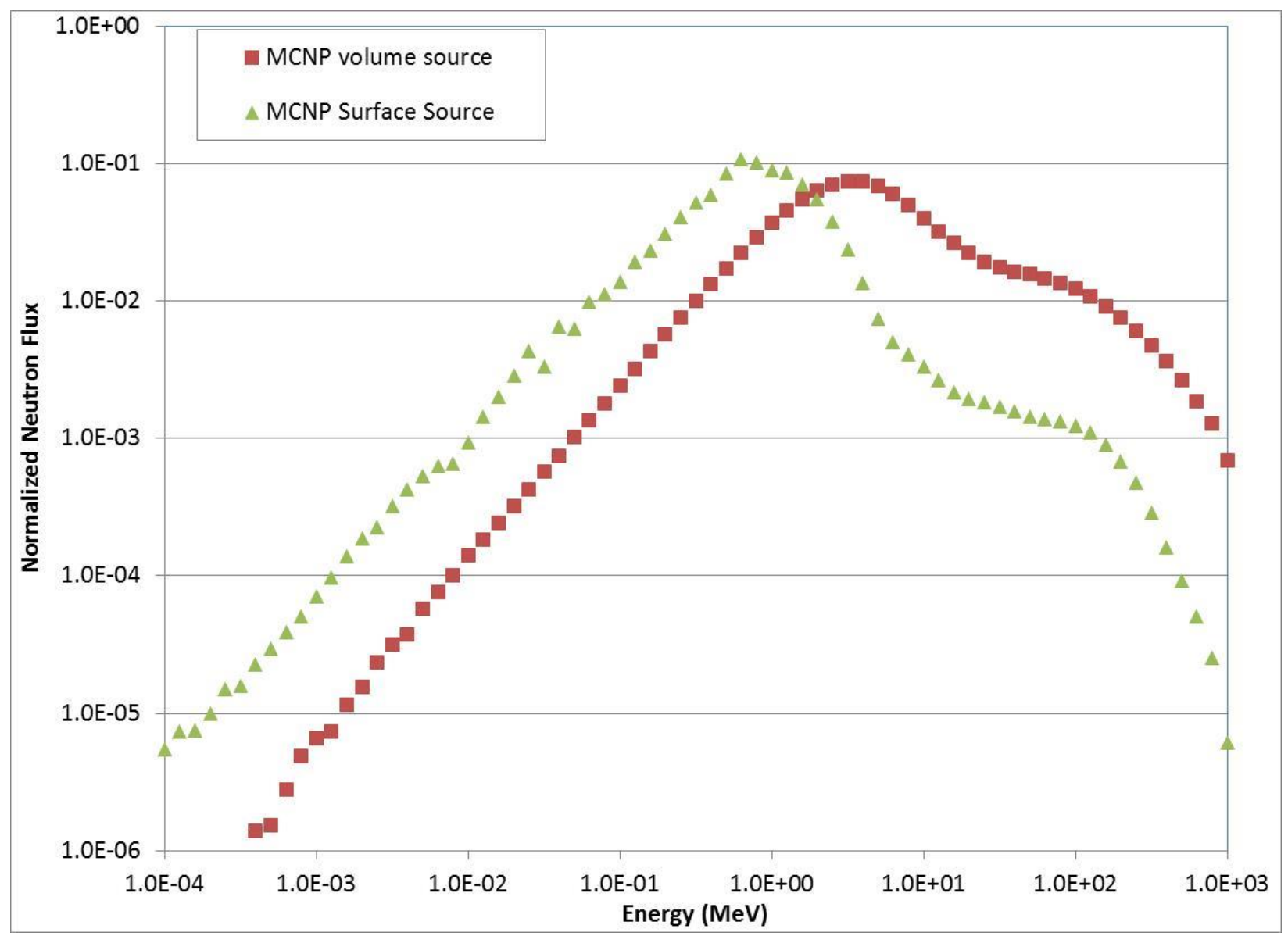

Figure 59 Energy spectrum of the spallation neturons at their born sites and at the outer target surface.

The generated neutron source files are used for the fuel burnup analyses of the different configurations. The MCNP6/MCNPX has a fuel burnup module, but it is only for critical reactors. The neutron transport equation is solved as a critical eigenvalue problem for the fuel burnup analysis. Subcritical systems are driven by external neutron sources using one of two scenarios. The first scenario adjusts the accelerator power to change the source strength for maintaining a constant fission power during operation. The second scenario keeps a constant accelerator power (i.e. the source strength) allowing the fission power to change during the burn cycle. For both scenarios, the neutron transport equation with an external source are solved for the burnup analysis. In addition, the effective multiplication factor is calculated at the beginning and the end of each fuel burnup step for fuel feeding purpose and safety consideration. To perform fuel burnup analysis of the ADS conceptual configurations, both the criticality transport equation and the fixed source transport equation need to be solved. Therefore, other Monte Carlo codes, MCB5 and SERPENT with such capabilities were used to perform the fuel burnup analyses for the different configurations [21]. The external neutron source files generated from MCNPX/MCNP6 are used for the MCB5 and the SERPENT burnup analyses. 
Both MCB5 and SERPENT codes are lacking the neutron cross sections for the highenergy neutrons. For neutrons with energy greater than the upper energy value of the cross section library, MCB5 uses the cross section values at the upper energy of the cross section library for transporting these high-energy neutrons. While SERPENT assumes zero cross section values (i.e. vacuum) for these high-energy neutrons. In this case, these neutrons escape from the system. In the current ENDF/B files, the majority of the cross section data files have maximum energy of $20 \mathrm{MeV}$, and some isotopes have cross section data up to $150 \mathrm{MeV}$. MCNP6/MCNPX have a physics models to calculate the cross section values for high-energy neutrons when the neutron energy exceeds the upper energy of the nuclear data files.

Before performing the burnup analyses, Monte Carlo simulations have been performed to calculate the neutron fluxes in the ADS bundle configuration driven by a point neutron source with different energies of 10,100 , and $300 \mathrm{MeV}$, respectively to examine the difference in the calculated neutron flux values by the three Monte Carlo codes. The MCNP6, the MCB5, and the SERPENT codes are utilized to calculate the total flux in the fission blanket respectively. For each calculation, the external point source is mono-energetic with isotropic distribution. Table XIV shows that both SERPENT and MCB5 codes underestimated the neutron fluxes by more than $30 \%$ for the case with 300 $\mathrm{MeV}$ external neutron source energy, despite the small differences observed at the low energy cases. In addition, the results for the $100 \mathrm{MeV}$ case are close to MCNP6 value because the cross section libraries for the main isotopes have cross section data up to $150 \mathrm{MeV}$.

In addition, the volume source generated from the MCNPX simulation was used to calculate the total fission energy in the blanket by the three Monte Carlo Codes for the bundle concept. The calculated SERPENT fission power with the volume source is 2.13 GW relative to $2.67 \mathrm{GW}$ calculated by the MCNP6, which is $20.2 \%$ less. With the surface source file, the calculated SERPENT fission power is $2.41 \mathrm{GW}$, which is $9.7 \%$ less than the MCNPX value. The calculated MCB5 fission power with the volume source is 2.14 GW, which is very close to the SERPENT value of $2.13 \mathrm{GW}$. Although the high-energy neutron fraction is small, it makes a significant difference in the total fission power and it shall be considered to account correctly for the total fission power. 
Table XIV. Comparison of the total neutron fluxes of the bundle conceptual configuration calculated with MCNP6, SERPENT, and MCB5 codes using point neutron source with different energies.

\begin{tabular}{|c|c|c|c|c|c|c|c|c|c|}
\hline \multirow{2}{*}{$\begin{array}{c}\text { Neutron } \\
\text { Source } \\
\text { Energy } \\
(\mathrm{MeV})\end{array}$} & \multicolumn{3}{|c|}{$\begin{array}{l}\text { MCNP6 } \\
\text { Flux (std) }\end{array}$} & \multicolumn{3}{|c|}{ MCB5 } & \multicolumn{3}{|c|}{ SERPENT } \\
\hline & $\begin{array}{c}\text { I } \\
\text { Physics } \\
\text { model } \\
\text { enabled }\end{array}$ & $\begin{array}{c}\text { II } \\
\text { Physics } \\
\text { model } \\
\text { disabled }\end{array}$ & $(\mathrm{I}-\mathrm{II}) / \mathrm{I}(\%)$ & Flux(std) & $\begin{array}{c}(\mathrm{I}-\mathrm{MCB} 5) / \mathrm{I} \\
(\%)\end{array}$ & $\begin{array}{c}(\text { II-MCB5)/II } \\
(\%)\end{array}$ & Flux(std) & $\begin{array}{c}(\text { I-SERP)/I } \\
(\%)\end{array}$ & $\begin{array}{c}(\text { II-SERP)/II } \\
(\%)\end{array}$ \\
\hline 10 & $\begin{array}{c}2.9214 \mathrm{E} 4 \\
(0.4 \%)\end{array}$ & $\begin{array}{c}2.9282 \mathrm{E} 4 \\
(0.4 \%)\end{array}$ & -0.23 & $\begin{array}{c}2.9732 \mathrm{E} 4 \\
(0.4 \%)\end{array}$ & -1.77 & -1.54 & $\begin{array}{c}2.9513 \mathrm{E} 4 \\
(0.5 \%)\end{array}$ & -1.02 & -0.79 \\
\hline 20 & $\begin{array}{c}\text { 4.8417E4 } \\
(0.3 \%)\end{array}$ & $\begin{array}{c}\text { 4.8494E4 } \\
(0.4 \%)\end{array}$ & -0.16 & $\begin{array}{c}4.8391 \mathrm{E} 4 \\
(0.4 \%)\end{array}$ & 0.05 & 0.21 & $\begin{array}{c}4.8215 \mathrm{E} 4 \\
(0.7 \%)\end{array}$ & 0.42 & 0.57 \\
\hline 100 & $\begin{array}{c}1.5509 \mathrm{E} 5 \\
(0.4 \%)\end{array}$ & $\begin{array}{c}1.5436 \mathrm{E} 5 \\
(0.6 \%)\end{array}$ & 0.47 & $\begin{array}{c}1.5193 \mathrm{E} 5 \\
(0.4 \%)\end{array}$ & 2.04 & 1.58 & $\begin{array}{c}1.4941 \mathrm{E} 5 \\
(0.7 \%)\end{array}$ & 3.67 & 3.21 \\
\hline 150 & $\begin{array}{c}2.1222 \mathrm{E} 5 \\
(0.5 \%)\end{array}$ & $\begin{array}{c}2.0989 \mathrm{E} 5 \\
(0.5 \%)\end{array}$ & 1.1 & $\begin{array}{c}2.0891 \mathrm{E} 5 \\
(0.4 \%)\end{array}$ & 1.56 & 0.47 & $\begin{array}{c}2.0642 \mathrm{E} 5 \\
(0.9 \%)\end{array}$ & 2.73 & 1.65 \\
\hline 200 & $\begin{array}{c}2.8651 \mathrm{E} 5 \\
(0.4 \%)\end{array}$ & $\begin{array}{c}2.3437 \mathrm{E} 5 \\
(0.5 \%)\end{array}$ & 18.2 & $\begin{array}{c}2.3388 \mathrm{E} 5 \\
(0.5 \%)\end{array}$ & 18.37 & 0.21 & - & - & - \\
\hline 300 & $\begin{array}{c}4.1643 E 5 \\
(0.4 \%)\end{array}$ & $\begin{array}{c}2.3480 \mathrm{E} 5 \\
(0.5 \%)\end{array}$ & 43.6 & $\begin{array}{c}2.3499 \mathrm{E} 5 \\
(0.5 \%)\end{array}$ & 43.57 & -0.08 & - & - & - \\
\hline
\end{tabular}


Monte Carlo fuel burnup analyses were performed for the ADS configuration using the bundle model. The fuel burnup results obtained with the MCB5 and SERPENT code were compared. The total fission power generated during the fuel burnup is maintained at $2.67 \mathrm{GW}$, which is the value calculated MCNP6 at the start up. As mentioned before, the fission power can be maintained at the specified value by varying the accelerator beam power or by adjusting the fuel composition to compensate for the reactivity losses. In MCB5 simulations, the fission power is $2.47 \mathrm{GW}$, which is the total prompt fission energy. In the SERPENT simulations, the fission power of $2.67 \mathrm{GW}$ is used since SERPENT uses the total fission $Q$ values for the fission power calculation.

The SERPENT code is used with the volume and surface source files in separate simulations to perform the fuel burnup analyses. The fuel slurry in the ADS conceptual configurations is slowly circulated. However, the fuel burnup analysis assumed stationary fuel material in the tubes and it is instantly mixed at the end of each fuel burnup step. The burnup time step is 90 days and fresh fuel particles are fed into the subcritical blanket at the end of each burnup step to compensate for the reactivity loss during the fuel burnup step. The amount of fresh fuel fed into the blanket each time step is estimated by using the same approach used in the previous analysis of the homogeneous preliminary design. The neutron transport equations are solved at the beginning and half way of each time step. The ENDF/B-VII.0 nuclear data were used in the simulations. Figure 60 shows the controlled keff values at the beginning and the end of each burnup step. Within each burnup step, the reactivity of the subcritical core decreases and it is brought back to the initial value of 0.98 at the beginning of the next burnup step.

The transmutation rates calculated with SERPENT from the two-burnup simulations are shown in Figure 61. The two simulations are identical except for the used neutron source file, the volume or the surface source. The results show little differences between the two simulations. The calculated fuel transmutation rates agree for each burnup step. This comparison shows that these high-energy neutrons have little impacts on the neutron spectrum inside the subcritical fission blanket. Both neutron source files can be used if the fission power is maintained at a constant power level during the fuel burnup analysis.

This configuration consumes 0.99 ton of actinides per year as shown in Figure 61 . The transmutation rate of MAs fuel peaks at the beginning and decreases slowly after the peak and it takes a very long time to reach an equilibrium rate. Meanwhile, the consumption of the plutonium fuel part shows an opposite behavior. If the ADS operates for 35 full power years, it will consumes 34.7 tons of actinides. Table XV list Serpent transmutation rates of the actinides using the ADS configuration operating for 35 full power years. The consumed MAs inventory during the 35 full power years is 25.1 tons. Therefore, 5.2 ADS units can consume the 131 tons of MAs of the US SNF.

In the ADS system, a fuel cleanup system is used to remove the short-lived fission products from the slurry fuel during the operation. However, in the fuel burnup analyses, all the fission products are carried through the life cycle of the subcritical system, except for the fission gasses ( $\mathrm{Kr}$ and $\mathrm{Xe}$ ) are removed at the end of each burnup step. Therefore, the actual performance of the ADS system will show small improvement relative to the 
calculated performance. Many of radioactive fission products have short half-lives, and need to be stored for few hundred years. However, the LLFP such as Se-79, Zr-93, Tc$99, \mathrm{Pd}-107$ and $\mathrm{l}-129$ have half-lives ranging from $10^{5}$ to $10^{7}$ years. The ADS concept can also transmute some of the LLFP to stable isotopes or isotopes with shorter half-lives. In the Monte Carlo fuel burnup analyses, the LLFPs are examined and their masses are calculated through the life cycle of the subcritical system.

Table XV. Serpent transmutation rates of the actinides using the ADS conceptual configuration operating for 35 full power years

\begin{tabular}{|c|c|c|c|c|}
\hline Fuel & $\begin{array}{c}\text { Initial } \\
\text { Inventory } \\
\text { (ton) }\end{array}$ & $\begin{array}{c}\text { Total Feed } \\
\text { (ton) }\end{array}$ & $\begin{array}{c}\text { Inventory at 35 } \\
\text { years (ton) }\end{array}$ & $\begin{array}{c}\text { Burnt/Transmuted } \\
\text { (ton) }\end{array}$ \\
\hline $\mathrm{U}$ & 0.076 & 0.109 & 1.08 & -0.895 \\
\hline $\mathrm{Pu}$ & 13.2 & 19.0 & 21.6 & 10.6 \\
\hline $\mathrm{MAs}$ & 14.7 & 21.1 & 10.7 & 25.07 \\
\hline $\mathrm{Np}$ & 4.89 & 7.04 & 2.96 & 8.97 \\
\hline $\mathrm{Am}$ & 9.65 & 13.9 & 6.48 & 17.06 \\
\hline $\mathrm{Cm}$ & 0.113 & 0.162 & 1.24 & -0.965 \\
\hline Total & 27.9 & 40.1 & 33.3 & 34.7 \\
\hline
\end{tabular}

Figure 62 shows the actual LLFPs inventory in the fuel slurry (solid curves), and the generated amount (dash curves). The Monte Carlo simulation demonstrated that the ADS system can transmute some LLFPs. Particularly, Table XVI lists the amount LLFPs transmuted during the ADS operation. After 35 years of fuel burnup, it is estimated that about $219 \mathrm{~kg}$ Tc-99, $63.2 \mathrm{~kg} \mathrm{l-129,} 0.76 \mathrm{~kg}$ Se-93, $20.4 \mathrm{~kg} \mathrm{Zr-93,} 160 \mathrm{~kg} \mathrm{Pd}-107$ and 178 $\mathrm{kg}$ Cs-135 have been transmuted in the fission blanket.

Table XVI Serpent calculated transmutation of the LLFPs in the ADS conceptual configuration using the bundle model within 35 years of operation.

\begin{tabular}{|c|c|c|c|}
\hline LLFP & $\begin{array}{c}\text { Produced } \\
\text { (ton) }\end{array}$ & $\begin{array}{c}\text { Inventory at 35 years } \\
\text { (ton) }\end{array}$ & $\begin{array}{c}\text { Transmuted } \\
(\%)\end{array}$ \\
\hline Tc-99 & 0.8135 & 0.5944 & $26.9 \%$ \\
\hline I-129 & 0.2552 & 0.1920 & $24.8 \%$ \\
\hline Se-79 & 0.005419 & 0.004659 & $14.0 \%$ \\
\hline Zr-93 & 0.4942 & 0.4737 & $4.1 \%$ \\
\hline Pd-107 & 0.5403 & 0.3803 & $29.6 \%$ \\
\hline Cs-135 & 1.424 & 1.246 & $12.5 \%$ \\
\hline
\end{tabular}

The slurry fuel compositions at different fuel burnup stages are show in Figure 63. At the startup, the fuel composition has 17.5\% Np-237, 29.7\% Pu-239, and 31.3\% Am241. During the fuel burnup, these isotopes are gradually fissioned or transmuted. After 35 years of burnup, the fuel composition is changed to $8.88 \% \mathrm{~Np}-237,17.2 \% \mathrm{Pu}-239$, and $15.2 \% \mathrm{Am}-241$. The fractions of Pu-238, Pu-240, and Pu-242 increase significantly to $16.7 \%, 19.9 \%$, and $8.3 \%$ respectively. The fraction of Am-243 and curium isotopes inside the slurry fuel remains small after 35 years of fuel burnup. Thus, the fuel burnup analysis 
shows that the ADS conceptual configuration consumes significant amount of Am among the MAs, without building too much amount of curium.

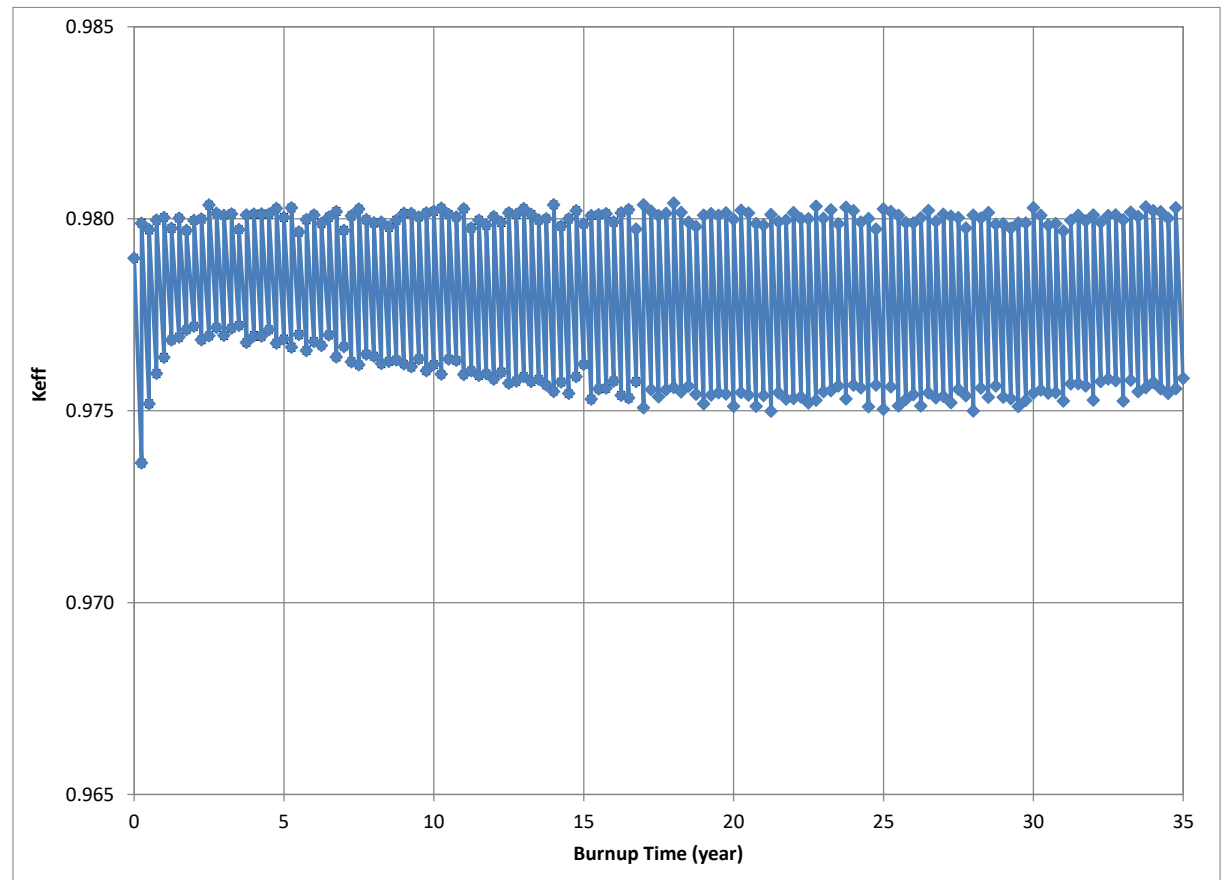

Figure 60 Calculated effective neutron multiplication values $\left(k_{\text {eff }}\right)$ before and after each fuel burnup time step of the ADS bundle configuration from SERPENT calculations using the surface neutron source.

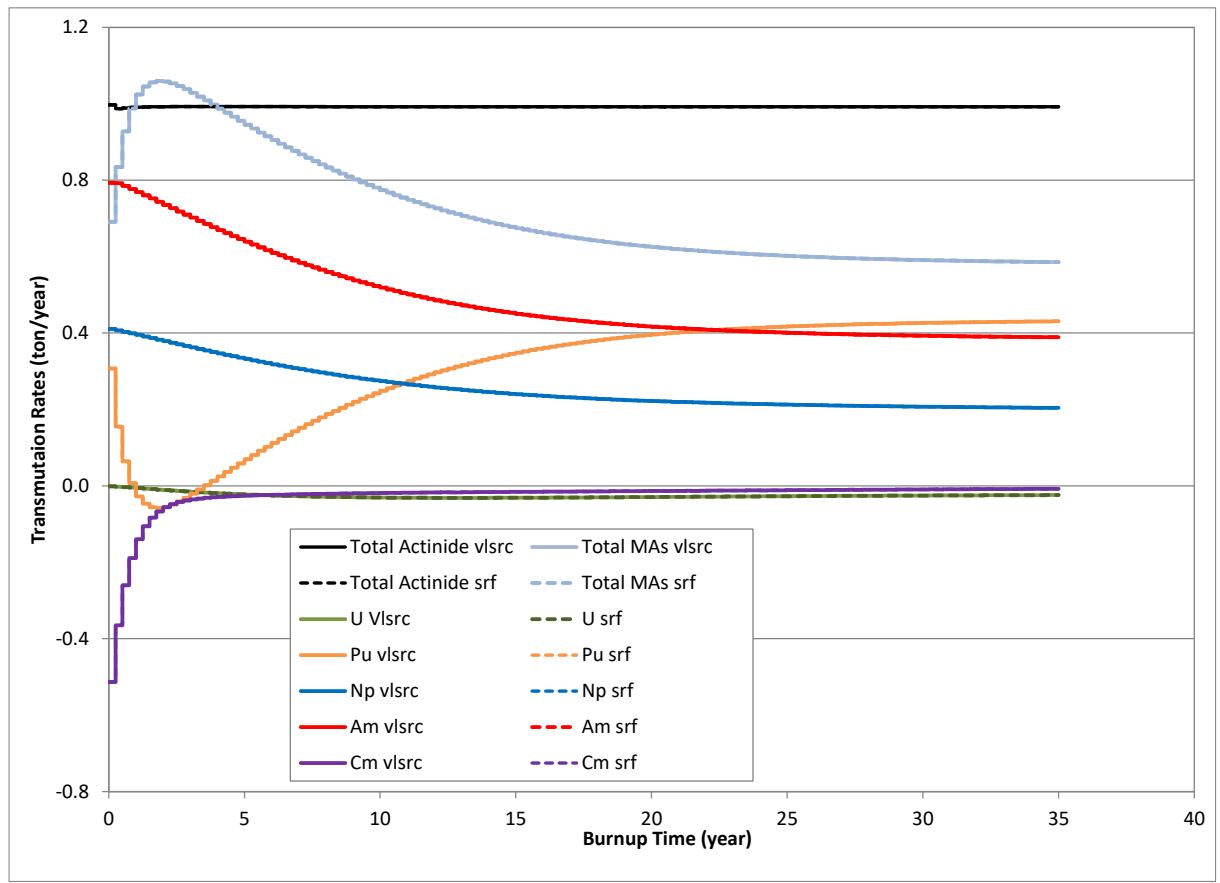

Figure 61 Actinide transmutation rates of the ADS bundle configuration from SERPENT calculations using the surface and the volume neutron source. 


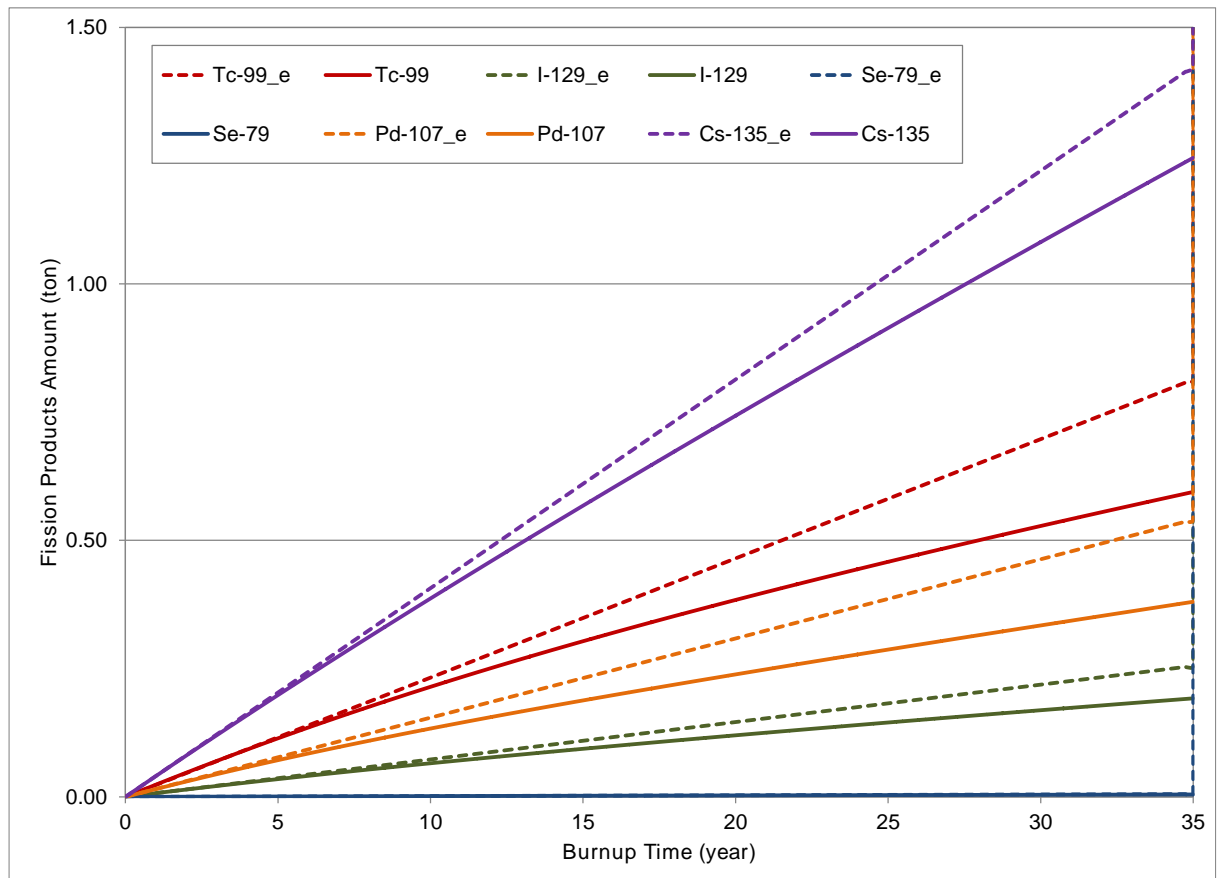

Figure 62 Generated and actual inventories of the long-lived fission products in the ADS mobile fuel as a function of the full power years.

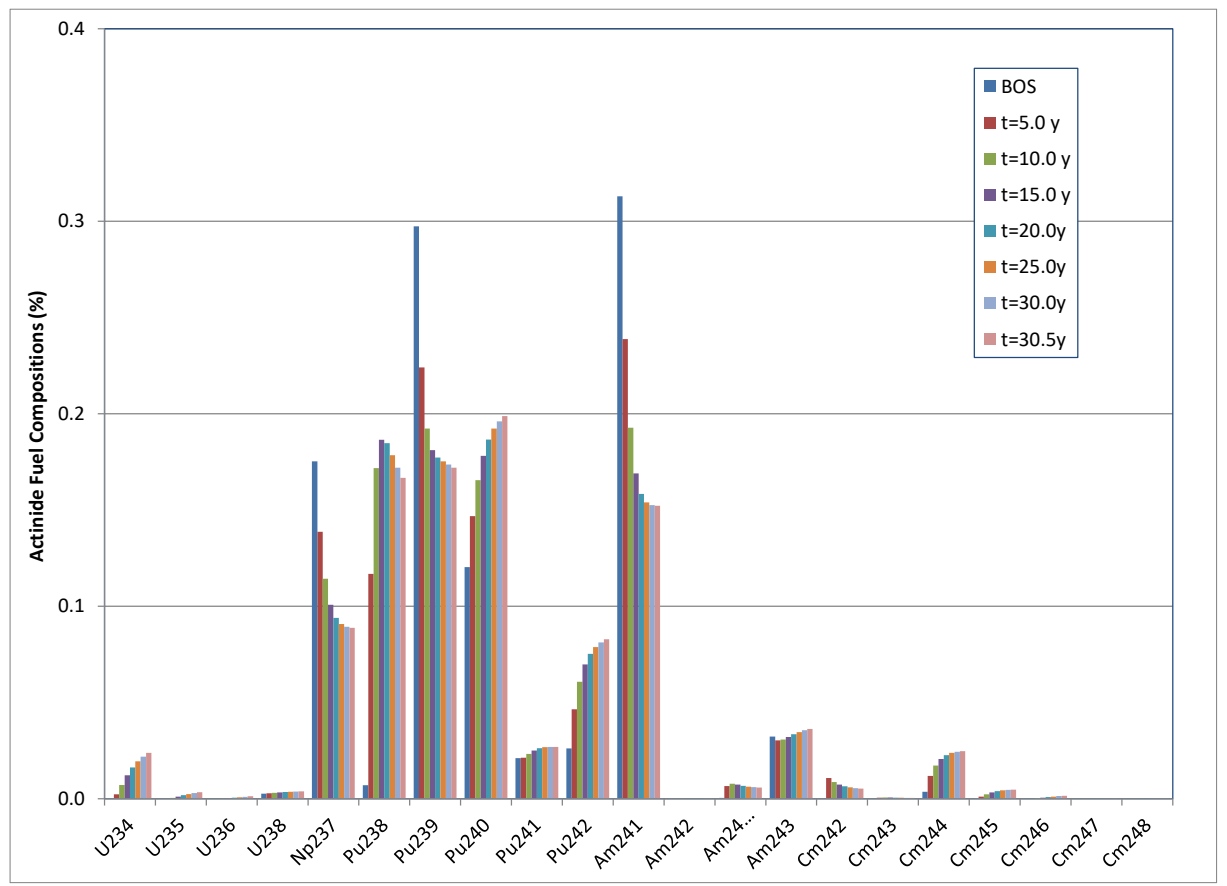

Figure 63 The actinide fuel compositions in the mobile fuel of the ADS bundle configuration after different operating periods.

Figure 64 shows the actinide fuel inventory and the total actinide fed into the subcritical fission blanket as a function of the full power years. The fuel inventory in the fission blanket decreases in the first few years of operation due to the conversion of the 
Am-241 fertile isotope to the Am242g/Am-242m fissile isotope. The calculational model does not account for the removal of the short-lived fission products from the slurry fuel, which requires extra fissile materials to compensate for the neutron losses to the fission products. Therefore, extra fresh fuel material is fed, and the fuel inventory gradually increases after the first few years of fuel burnup. For future analysis, the fission products removal procedure will be integrated in the calculational model, which will reduce the feeding amount. Then, the actinide fuel inventory will not change after a few full power years of operation.

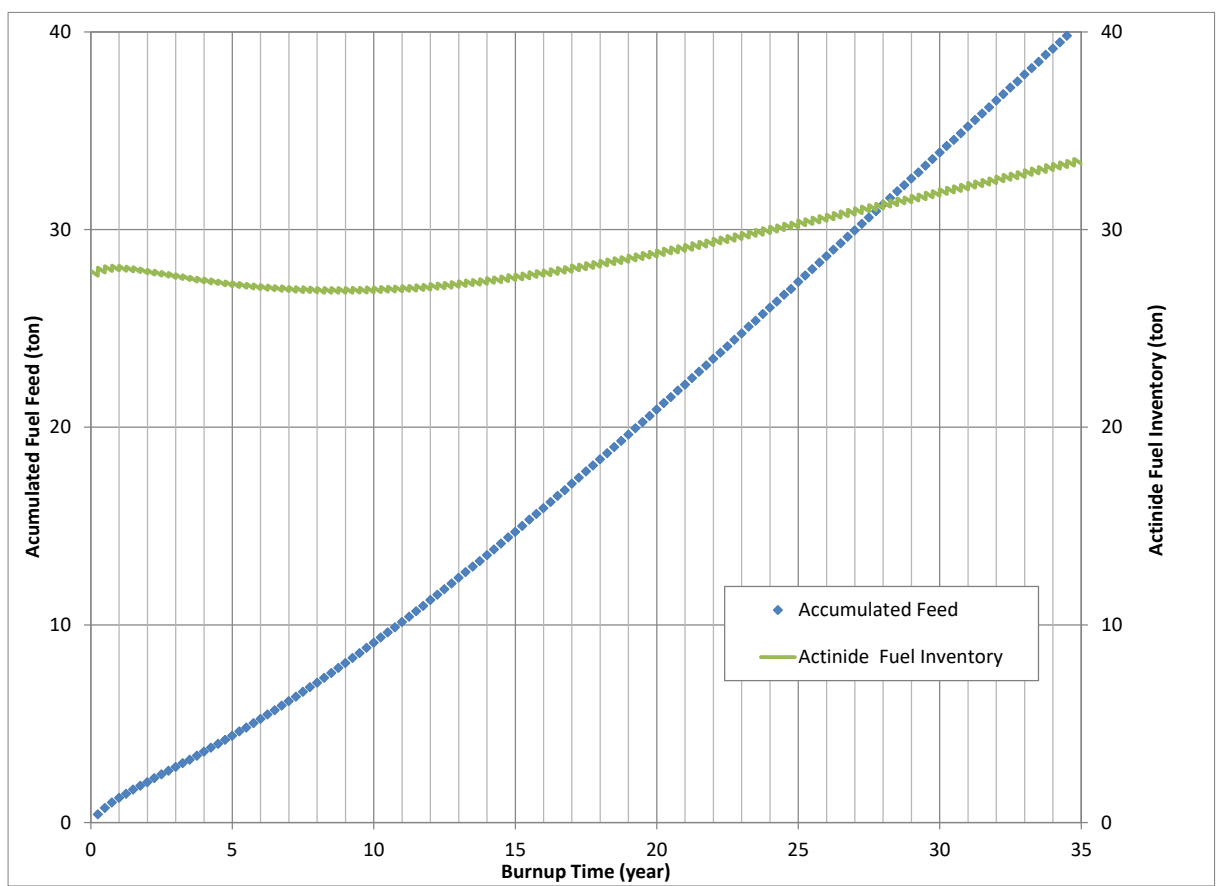

Figure 64 The actinide fuel inventory and the total actinide fed into the subcritical fission blanket as a function of the full power years.

In addition, MCB5 Monte Carlo fuel burnup analyses were performed with the ADS bundle configuration. The volume source was used for the analyses. The fission products except the noble fission gasses were not removed during the ADS operation similar to SERPENT analyses. The time step of the fuel burnup is 90 days and the ENDF-B/VII data were used. MCB5 utilizes the released prompt kinetic energies of the fission fragments, the fission neutrons, and the decay heat from the ORIGEN libraries (gammas and betas) for calculating the fission power. The fission power used for each burnup step is $2.47 \mathrm{GW}$, which match the total power used in the SERPENT burnup analyses.

The actinide transmutation rates from the MCB5 and the SERPENT codes are shown in Figure 65. Both codes calculate the annual transmutation rate of the total actinide fuel and the calculated value is $\sim 0.99$ ton/year. The MCB5 code calculates a lower transmutation rate for $\mathrm{Am}$, a higher transmutation rate for $\mathrm{Pu}$, and a slightly lower accumulation rate for $\mathrm{Cm}$ isotopes. The transmuted amount of the Am isotopes, Pu242, and $\mathrm{Cm} 242$ are plotted in Figure 66 as a function of the full power years for the first four 
years. Both codes transmute the same amount of Am241, however the MCB5 code generates more Am242m and slightly less Pu242 and Cm242.

The difference in the calculated transmutation rates from the two codes for the Am and $\mathrm{Pu}, \mathrm{Cm}$ is due to the different branching ratios of Am241 ( $\mathrm{n}$, gamma) reaction. As illustrated in the previous analysis before, the Am241 absorbs a neutron and becomes either Am242g at ground state or Am242m. The Am242g has a half-life of only 16 hours and quickly decay to Pu242 and Cm242. The Am242m has a half-life of around 141 years and decays to Am242g. The simulation results indicate that the MCB5 burnup calculation of the Am241 ( $\mathrm{n}$, gamma) reaction produces more Am242m than that of SERPENT. The Am242m has a longer half-live and it is a fissile isotope.

The controlled $k_{\text {eff }}$ values and the integrated amount of the actinide fuel fed into the fission blanket calculated by both codes as a function of the operating full power years are compared in Figure 67. In MCB5 results, the extra Am242m production results in smaller reactivity losses in each burnup step compared with the SERPENT results in the first few full power years of operation. In addition, less integrated amount of fresh fuel is required to control the $k_{\text {eff }}$ of the fission blanket as shown in the figure 67 . In the recent nuclear data files, the branching ratio of $A m 241$ ( $n$, gamma) reaction is a function of the neutron energy as shown in Figure 6. The branching ratio in MCB5 was updated using ENDF/B-VII.O data files. In SERPENT code, this branching ratio is a fixed number of 0.885 to produce $A m 242 \mathrm{~g}$, which significantly underestimates the production of Am242m from high-energy neutrons.

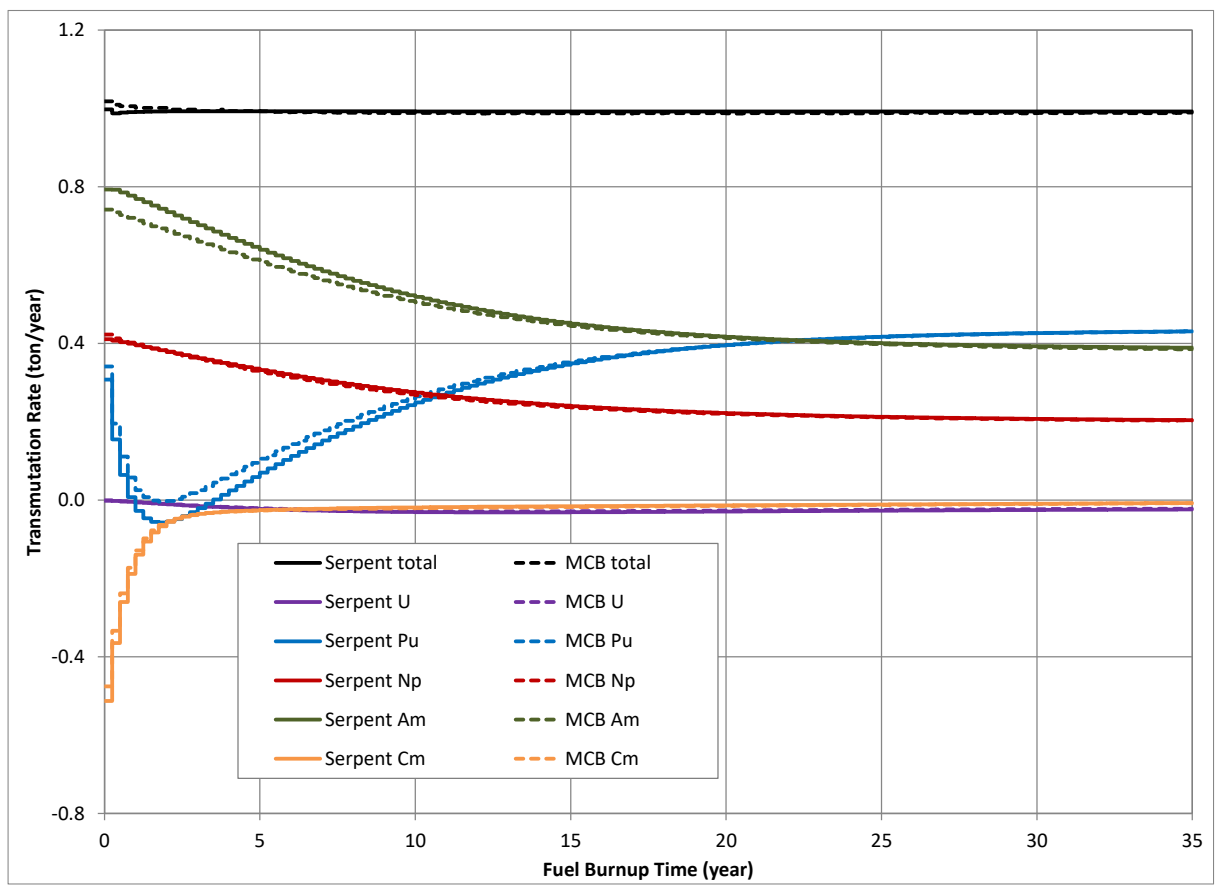

Figure 65 Comparison of the calculated transmutation rates from the SERPENT and the MCB5 simulations using the volume source for the ADS bundle configuration. 


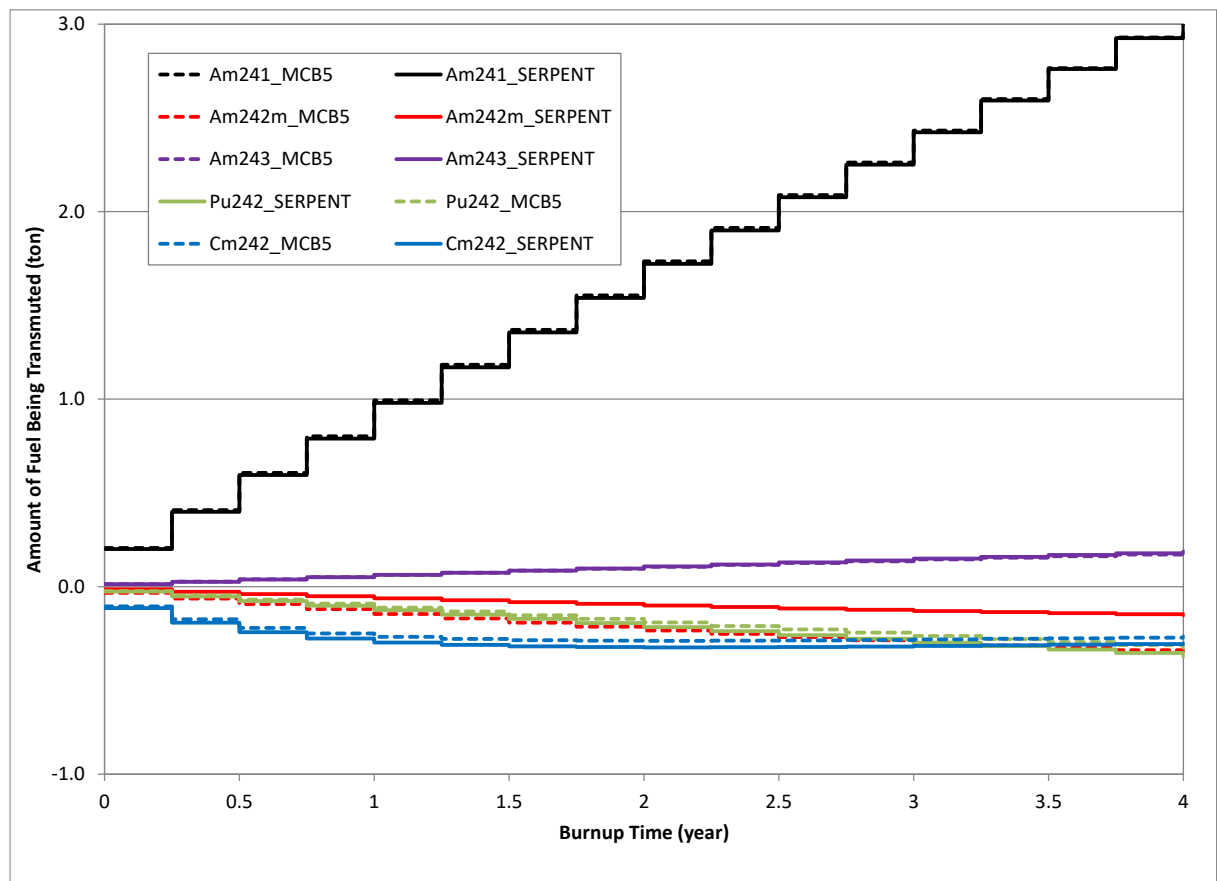

Figure 66 Comparison of the consumed actinides calculated from the SERPENT and the MCB5 simulations using the volume source for the ADS bundle configuration.

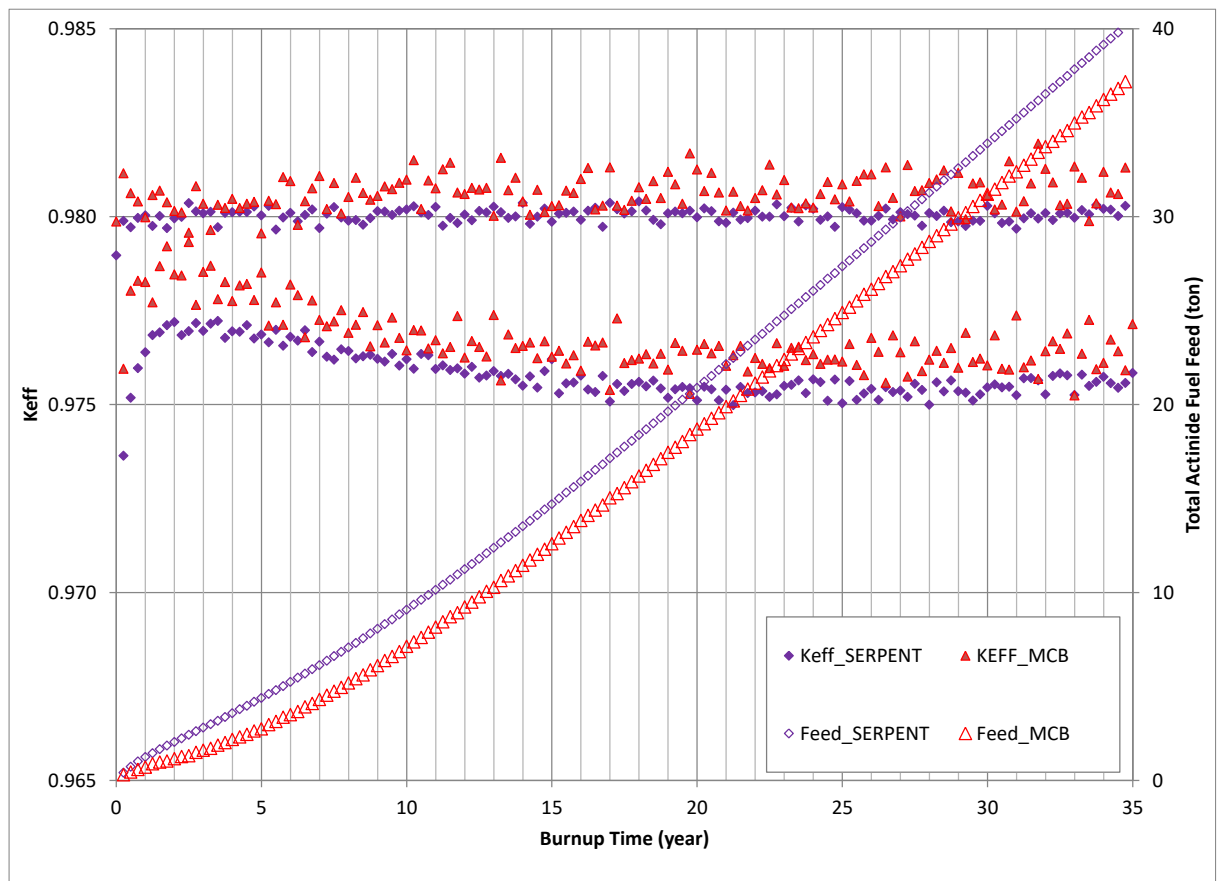

Figure 67 Comparison of the controlled $k_{\text {eff }}$ and the integrated amount of actinide fuel fed from the SERPENT and the MCB5 simulations using the volume source for the ADS bundle configuration.

Table XVII compares the transmuted actinides per an ADS bundle system operating for 35 full power years from SERPENT and MCB5 simulations. The MCB5 simulation 
shows that the ADS configuration transmutes 16.6 tons of $\mathrm{Am}, 8.87$ tons of $\mathrm{Np}$, and 11.0 tons of $\mathrm{Pu}$. At the same times, it produces 0.97 tons of $\mathrm{Cm}$. To consume the 131 tons of MAs among of the current US SNF inventory, 5.3 ADS systems are required.

Table XVII. Transmuted actinides from the MCB5 and the SERPENT simulations of the ADS bundle configuration operating for 35 full power years.

\begin{tabular}{|c|c|c|}
\hline Element & SERPENT Simulation, tons & MCB5 Simulation, tons \\
\hline $\mathrm{U}$ & -0.90 & -0.828 \\
\hline $\mathrm{Pu}$ & 10.57 & 10.976 \\
\hline $\mathrm{MAs}$ & 25.05 & 24.491 \\
\hline $\mathrm{Am}$ & 8.97 & 8.871 \\
\hline $\mathrm{Cm}$ & 17.05 & 16.587 \\
\hline Total Actinide & -0.96 & -0.968 \\
\hline
\end{tabular}

The LLFPs produced from the fission reactions obtained from the MCB5 and the SERPENT simulations are compared in Figure 68 as a function of the operating time for 35 full power years. MCB5 has a build in fission yield library while SERPENT uses the fission yield library from the ENDF/B-VII data files. Therefore, the calculated production rates of the fission products from the two codes are different. The MCB5 simulations predict a higher amount of $\mathrm{Zr}-93, \mathrm{I}-129$ and a lower amount of $\mathrm{Pd}-107$ compared with the SERPENT simulations.

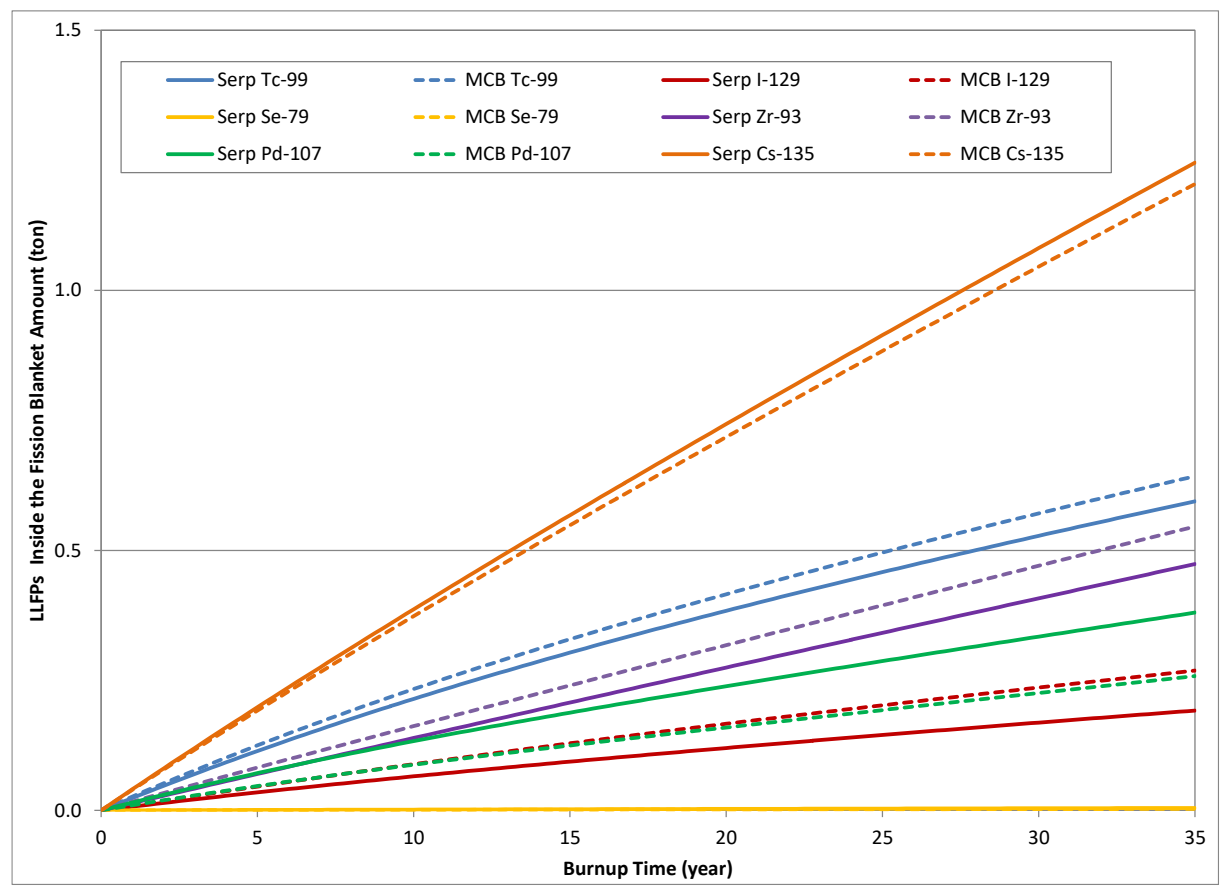

Figure 68 Comparison of the LLFPs from the SERPENT and the MCB5 simulations of the ADS bundle configuration using the volume source and operating for 35 full power years. 


\section{Summary}

This report examined ADS systems for disposing of the US SNF by transmuting the 131 metric tons of MAs of the current 80,000 metric tons of US SNF inventory. The ADS neutron source is a spallation source with a $25-\mathrm{MW} 1-\mathrm{GeV}$ proton beam striking a liquid metal target, i.e., lead-bismuth eutectic (LBE) target in the preliminary analysis, or liquid lead target in the later systems. The fission blanket deployed the mobile fuel concept. The same liquid metal used of the neutron target is used as the fuel carrier. The actinide fuel materials are dissolved, or suspended as micro particles in the liquid fuel carrier to form the fuel slurry.

Preliminary analyses were first carried out with a simple homogeneous configuration, which has a LBE target at the center, a cylindrical fission blanket outside the target region filled with the fuel slurry, and a graphite reflector outside the fission blanket. Steady-state Monte Carlo simulations using the MCNPX code were performed to determine the overall system parameters. The source region geometry was first determined to satisfy the energy deposition limit imposed on the target window and the surface temperature limit of the structure materials. The neutron multiplication factor $k_{\text {eff }}$ of the fission blanket was determined to be approximately 0.98 so that the $25 \mathrm{MW}$ proton beam can produce about $3 \mathrm{GW}$ of fission power within the homogeneous blanket. The fuel composition used in the mobile fuel slurry was based on the composition of the LWR discharged fuel. The fraction of plutonium inside the actinide fuel was adjusted to achieve $0.98 \mathrm{k}_{\text {eff. }}$ The total amount of the plutonium material in the initial fuel inventory was kept small as possible to burn more MAs. Three possible configurations for the fission blanket with 5,7 , and $10 \%$ actinides in LBE were defined based on the results from the Monte Carlo steady state simulations with $0.98 \mathrm{k}_{\text {eff }}$ and $\sim 10$ tons of MAs initial inventory.

Monte Carlo fuel burnup simulations using the MCB5 code were performed to analyze the performance of the three configurations with ENDF/B-VII.0 nuclear data libraries. In the MCB5 burnup calculations, the Am-241 neutron capture branching ratio to Am-242m has also been updated using the updated values from the ENDF/B-VII.0 nuclear files. To simulate the continuous operation of the system, fresh fuels are fed into the fission blanket to adjust its reactivity at the end of each burnup step to the $0.98 \mathrm{k}_{\text {eff }}$ and to control the system power. The burnup time step is 90 days, with fission power fixed at $3 \mathrm{GW}$ during each burnup step.

According to the MCB5 burnup results, the three configurations consume about 1.2 tons of actinides per year. The annual transmutation rate of MAs is slightly higher with more actinides in the LBE. Total MAs weights consumed during the first 10 years of operation are $9.85,11.80$, or 12.68 tons for the configurations with 5,7 , or $10 \%$ actinides in the LBE, respectively. The corresponding annual MAs fuel transmutation rates are $0.83,094$, and 1.02 tons/year for the three configurations, respectively, after reaching equilibrium at 10 burnup years. Therefore, the total MAs weights burned by the three configurations are $30.6,35.3$, and 37.2 tons, respectively, assuming that the ADS system can be operated for 35 years. To utilize the 131 tons of MAs from the 80,000 tons of SNF, 
only 4.3, 3.7, or 3.5 ADS systems are required for the three configurations considered in this preliminary study, respectively.

Then, the spallation neutron source examined the liquid lead as the target material. The reactor physics and thermal-hydraulic calculations were coupled to define the target configuration. The maximum surface temperature of the steel structure material is limited to less than $600{ }^{\circ} \mathrm{C}$ and the maximum liquid metal velocity is restricted to less than $3 \mathrm{~m} / \mathrm{s}$. In addition, the liquid lead is used as the mobile fuel carrier in designing the fission blanket and three design configurations were studied. The homogenous self-cooled configuration was eliminated due to the large mobile fuel inventory and other concerns. Two configurations using separate primary cooling loops with bundle tubes inserted into the subcritical assembly tank were considered.

The inverted bundle model with primary molten lead coolant inside the tubes was first studied. The coolant tubes are arranged in cylindrical rings. These tubes are connected outside to six separate cooling loops to remove the fission heat. The slurry fuel is slowly circulated inside a cylindrical tank and only a small stream is taken outside the fission blanket for fuel chemistry control. The stainless steel reflector is used to avoid the fission power peaking at the blanket reflector interface. The thermal-hydraulic analyses were coupled with the physics analyses and iterated to define the coolant tube positions. Two configurations with the tube diameter of $1.4 \mathrm{~cm}$ or $1.7 \mathrm{~cm}$ were defined with a satisfactory performance. The maximum surface temperature of the steel structure is less than $600{ }^{\circ} \mathrm{C}$, and the maximum liquid lead velocity is less than $3 \mathrm{~m} / \mathrm{s}$. For both cases, the fission blanket has $4 \mathrm{~m}$ diameter and $4 \mathrm{~m}$ height with $0.69 \mathrm{~m}$ target diameter at the center. The total fission power is $\sim 2.5 \mathrm{GW}$ with the $0.98 \mathrm{k}_{\text {eff }}$ and the $25-\mathrm{MW} \mathrm{1-GeV}$ proton beam. To compensate for the neutron losses in the tube structure material, the slurry fuel contains $\sim 8 \%$ actinides for both configurations and the plutonium concentrations in the actinides is slightly more than $46 \%$. The initial actinide inventory is $\sim 23$ tons with $\sim 12.5$ tons of MAs. The inverted bundle configurations have 13824 or 18336 coolant tubes. These coolant tubes are long and thin, which represent an engineering challenging to fabricate and to operate safely.

A different bundle model with the slurry fuel inside the tubes was then developed to use larger tubes and to reduce the number of the tubes in the fission blanket. The fuel tubes have hexagonal arrangement connected to each other from the top and bottom. The slurry slowly circulates inside the fuel tubes and a small stream is taken outside the fission blanket for chemistry control, removal of the short-lived fission products, and fuel feeding. The fission blanket is filled with the primary lead coolant and it is divided into six sectors. Each sector is connected to a separate cooling loop outside the fission blanket. The reactor physics and the thermal-hydraulic analyses were iterated to determine the size of the fuel tubes. An ADS configuration was defined, which satisfies the designed requirements and is easier to be fabricated. This configuration uses larger tubes with diameter ranges from $2.3 \mathrm{~cm}$ to $2.9 \mathrm{~cm}$. The number of tubes is significantly reduced. The total fission power is $\sim 2.67 \mathrm{GW}$ with the $0.98 \mathrm{k}_{\text {eff }}$ and the 25-MW 1-GeV proton beam. The dimensions of the fission blanket cylinder are $4.4 \mathrm{~m}$ diameter and $4.4 \mathrm{~m}$ height. The initial fuel actinide fuel inventory is $~ 27.4$ tons, including 14.0 tons of MAs. 
Monte Carlo burnup analyses were performed with the SERPENT and the MCB5 codes for the ADS configurations. Fresh fuel particles were added at the end of each burnup step to keep $k_{\text {eff }}$ value at 0.98 . The burnup time step is 90 days and the fission power is $2.67 \mathrm{GW}$. The SERPENT and the MCB5 burnup results show that the consumed actinides are 0.99 and 0.96 tons per year, respectively. Assuming 35 full power years per ADS system, the utilized MAs are 25.1 and 24.5 tons based the SERPENT and the MCB5 results, respectively. This means that $\sim 5.3$ ADS systems can utilize the 131 tons of MAs of the 80,000 tons of the US SNF inventory. In addition, a significant amount of LLFPs will be transmuted at the same time. This show that the ADS system is an effective approach for disposing of the US SNF inventory. 


\section{References}

[1] DOE Office of Civilian Radioactive Waste Management, "Draft Environmental Impact Statement for a Geologic Repository - Appendix A: Inventory and Characteristics of Spent Nuclear Fuel, High-Level Radioactive Waste, and Other Materials," ODE/EIS0250D, July 1999.

[2] R. N. Hill, "LWR Feed Inventory Specification for ATW System Studies," INTRALABORATORY MEMO, June 2000.

[3] DOE Office of Civilian Radioactive Waste Management, "A Roadmap for Developing Accelerator Transmutation of Waste (ATW) Technology - A Report to Congress," DOE/RW-0519, Oct. 1999.

[4] The European Technical Working Group on ADS System, "A European Roadmap for Developing Accelerator Driven Systems (ADS System) for Nuclear Waste Incineration," April 2001.

[5] Nuclear Energy Agency, OECD, "Physics and Safety of Transmutation Systems, A Status Report," ISBN 92-64-01082-3, 2006.

[6] W. Maschek, X. Chen, F. Delage, A. Fernandez-Carretero, D. Haas, C. Matzerath Boccaccini, A. Rineiski, P. Smith, V. Sobolev, R. Thetford, and J. Wallenius, "Accelerator Driven Systems for Transmutation: Fuel Development, Design and Safety," Prog. Nucl. Eng., 50, 333-340, 2008.

[7] K, TSUJIMOTO, T. SASA, K. NISHIHARA, H. OIGAWA, and H. TAKANO, "Neutronics Design for Lead-Bismuth Cooled Accelerator-Driven System for Transmutation of Minor Actinide," J. Nucl. Sci. Tech., 41, 21-26, 2004.

[8] H. T. HAHN, "Uranium Oxide - Liquid Metal Slurries," Industrial and Engineering Chemistry, 51, 2, 197-199, 1959.

[9] B. Becker, M. Fratoni, and E. Greenspan, "Feasibility of a Critical Molten Salt Reactor for Waste Transmutation," Prog. Nucl. Eng., 50, 236-241, 2008.

[10] Y. Gohar, P. J. Finck, L. Krajtl, J. E. Herceg, W. D. Pointer, J. Saiveau, T. Sofu, A. L. Hanson, M. Todosow, M. Koploy, and P. Jijatovic, "Lead-Bismuth Target Design for the Subcritical Multiplier (SCM) of the Accelerator Driven Test Facility (ADTF)," ANL/TD/02-01, Argonne National Laboratory, Jan. 2002.

[11] D. B. Pelowitz, "MCNPX User's Manual, Version 2.6.0," LA-CP-03-1473, April 2008.

[12] J. Cetnar, W. Gudowski, and J. Wallenius, "User Manual for Monte-Carlo Continuous Energy Burnup (MCB5) Code - Version 1C."

[13] J. Leppänen, "Serpent - a Continuous-energy Monte Carlo Reactor Physics Burnup Calculation Code, User's Manual," June 18, 2015.

[14] CD-Adapco, "User Guide, STAR-CCM+ 8.04," 2013.

[15] S. Andriamonje, et al., "Experimental determination of the energy generated in nuclear cascades by a high energy beam," Phy. Letter B, 348, 697-709, 1995.

[16] OECD/NEA Nuclear Science Committee, "Handbook on Lead-bismuth Eutectic Alloy and Lead Properties, Materials Compatibility, Thermal-hydraulics and Technologies," NEA No. 61952015 (227).

[17] Alloy Phase Diagram Center, http://www1.asminternational.org/asmenterprise/apd.

[18] L. Golyand, E. Shwageraus, and Y. Ron, "Effect of ${ }_{241}^{95} \mathrm{Am}(\mathrm{n}, \mathrm{g})$ Reaction Branching Ratio on Fuel Cycle and Reactor Design Characteristics," Nucl. Sci. Eng.,161,289302, 2009. 
[19] M. B. Chadwick, et al., "ENDF/B-VII.0: Next Generation Evaluated Nuclear Data Library for Nuclear Science and Technology," Nuclear Data Sheets, 107, 2931-3060, 2006.

[20] F. M. Mann and R. E. Schenter, "Calculated Neutron Capture Cross Sections to the Americium Ground and Isomeric States," Nucl. Sci. Eng., 63, 242-249, 1977.

[21] Y. Cao and Y. Gohar, "Conceptual Design of a Minor Actinides Burner with an Accelerator-Driven Subcritical System," ANL-11/34, September 2011.

[22] W. Pfrang and D. Struwe, "Assessment of Correlations for Heat Transfer to the Coolant for Heavy Liquid Metal Cooled Core Designs," FZKA 7352 (2007).

[23] N. Todreas and M. Kazimi, "Nuclear Systems I: Thermal Hydraulic Fundamentals," Taylor \& Francis (1990). 


\section{Argonne}

Nuclear Science \& Engineering Division

Argonne National Laboratory

9700 South Cass Avenue, Bldg. *\#

Argonne, IL 60439

www.anl.gov 